\title{
DIRECT CONVERSION OF RADIOISOTOPE ENERGY TO
}

\section{ELECTRICITY: Year 3 Report}

DOE DE FG07-001D13927

University of Missouri-Columbia

E2435 Engineering Bldg. East

Nuclear Engineering

Columbia, MO 65211

Mark A. Prelas, PI 


\section{TABLE OF CONTENTS}

\section{DIRECT CONVERSION OF RADIOISOTOPE ENERGY TO ELECTRICITY: Year 3}

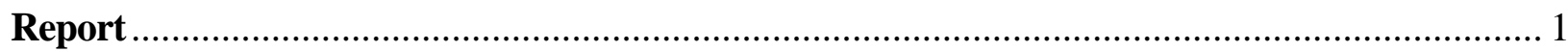

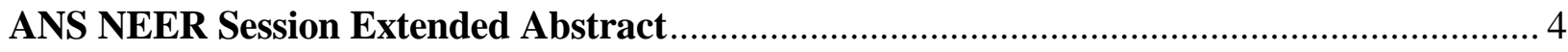

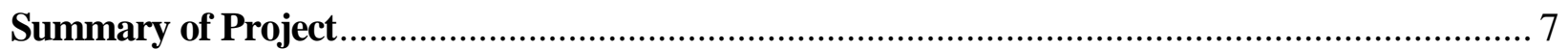

Accomplishments Under Each Objective ....................................................................... 16

1) Characterization of excimer fluorescence sources (e.g., $\mathrm{Kr} 2 *-8.4 \mathrm{eV}$ photon)...................... 16

1a) Build an excimer fluorescence source powered by microwaves for spectroscopy. A cell will be designed which can test VUV-Visible excimers (Year 1 Objective, Duration- 8 months).......... 16

1.b) Absolutely calibrate spectra from the excimer fluorescers (Year 1 Objective, Duration- 3

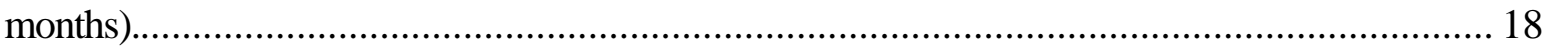

1.c) Determine efficiencies for the production of excimer fluorescence (Year 1 Objective,

Duration- 2 months)

2) Develop photovoltaic cells that have band-gaps, which match the bandwidths of the excimer photon sources (Diamond - $5.5 \mathrm{eV}$, AlN - $6.2 \mathrm{eV}$ ).

2.a) Obtain polycrystalline or monocrystalline diamond (Year 1 Objective, Duration- 1 month). 21

2.b) Obtain polycrystalline or monocrystalline AIN (Year 2 Objective, Duration- 1 month)... 22

2.c) Form junctions in diamond (Year 2 Objective, Duration-12 months )............................. 22

2.d) Form junctions in AlN (Year 3 Objective, Duration- 12 months)................................... 24

2.e) Characterize surface of diamond (Year 1 Objective, Duration- 2 months)................... 25

2.f) Characterize surface of AlN (Year 2 Objective, Duration- 2 months). ......................... 26

2.g) Characterize the optical performance of the diamond photovoltaic cells (Year 2 Objective,

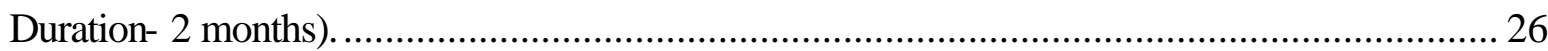

2.h) Characterize the optical performance of the AlN photovoltaic cells (Year 3 Objective,

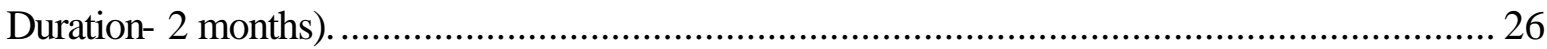

2.i) Develop antireflection coatings cells (Year 3 Objective, Duration- 4 months)............... 30

3) Build single tests cell electrical generator using the excimer and the photovoltaic cell, which best matches its bandwidth (Year 1 Objective, Duration 3 months).

3.a) Design a system to model a radioisotope driven excimer lamp using a discharge lamp and optical coupling system (Year 3 Objective, Duration 1 month).

4) Develop models for the transport of ions, the transport of photons and the transport of ionizing radiation. This will guide the experimental aspects of the project as well as predict scaling of the portable power generators (Year 1 Objective, Duration- 6 months---Year 2 Objective, Duration 6

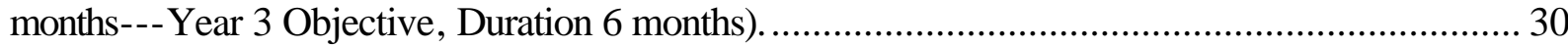

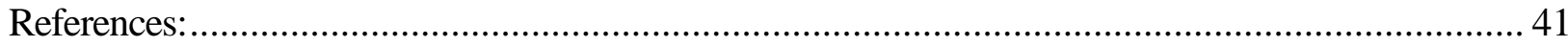

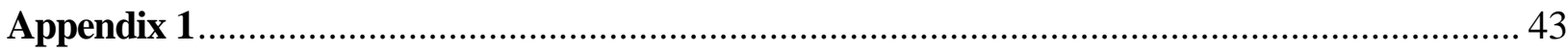


As part of the reporting process for year 2, the results from the second year of DOE DE FG07-

001D13927 were presented at the NEER session at June 2002 ANS meeting The slide presentation is attached as Appendix 1. 


\title{
ANS NEER Session Extended Abstract
}

\section{Wide Band-Gap Photovoltaics for Nuclear Energy Conversion}

\author{
Mark A. Prelas, Alexey Spitsyn, Alejandro Suarez, \\ Eric Stienfelds, Dickerson Moreno, Bia-Ling Hsu, \\ Tushar K. Ghosh, Robert V. Tompson, Sudarshan K. \\ Loyalka and Dabir Viswanath \\ University of Missouri-Columbia
}

\section{Abstract}

A new chemical reactor has been tested for Field Enhanced Diffusion by Optical Activation (FEDOA) doping and purification of SiC, GaN and AlN films. Different conditions have been used on SiC, GaN and AlN samples including temperature variation, electrical field variation, variations in electrical current and optical activation. A $5 \mathrm{~mW}(630-680 \mathrm{~nm})$ laser was used for optical activation. It was observed that optical activation has a major effect on ion drift rates. It was also observed that the magnitude of the electrical current also enhanced ion drift rates by a postulated current drag mechanism. I-V characteristic curves were measured to verify changes in the electrical properties of the samples. SIMS was used to analyze the concentrations of impurities in the film samples before and after treatment. It has been demonstrated that the fieldenhanced diffusion by optical activation method can dope and purify the films. As a result, the electrical properties of the wafers have been significantly improved during treatment especially in cases where a laser is used.

\section{Introduction}

Energy conversion of the radiation emitted from radioisotopes to electricity can be achieved using an intermediate step that creates ultraviolet photons. These ultraviolet photons can be efficiently transported to a transducer to produce electricity. This process is known as the Radioisotope Energy Conversion System (RECS) [1, 2]. The advantage of the RECS is high-energy conversion efficiency 30\% to 50\% [3]. One of the keys to the concept is the development of a transducer that converts ultraviolet photons to electricity. This paper discusses the development of wide band-gap photovoltaic cells. 
The FEDO process, developed by the authors, uses a combination of temperature, electric field, optical field and current density to move impurities into (or out of) wide band-gap materials. Wide band-gap materials are extremely difficult to dope and FEDO is one of the few processes that has been demonstrated for this purpose [4]. The goal is to produce a p-n junction in SiC, GaN, and AlN. Both SiC and GaN are currently being used as light emitting diodes, the FEDO process is able to remove unwanted impurities in the wafer before processing [4]. Additionally, FEDO represents an alternative means of producing a $\mathrm{p}-\mathrm{n}$ junction in SiC and GaN. FEDO has been used, for the first time, to add impurities to SiC and GaN.

A $\mathrm{p}-\mathrm{n}$ junction has yet to be formed in AlN which is the most desirable material for use as a transducer due to the good match up of its band-gap with the ultraviolet photon energy. The FEDO process was used to dope AlN with carbon, silicon, and magnesium to form $\mathrm{p}$ and $\mathrm{n}$ type semiconductor. FEDO has been successful in the addition of these impurities to AlN and a good first step to the formation of an AlN p-n junction.

The ultimate question of the conversion efficiency of the photovoltaic cells will depend upon the quality of the material and of the defect density.

Bibliography

1. E. V. Stienfelds, T. Ghosh, M. Prelas, R.V. Tompson and S. Loyalka, Computer Simulations of Novel RECS Designs Plus Experimental Development, Trans. American Nuclear Soc., V85, (ISSN: 003-018X), pp 309-310 (2001).

2. E. V. Steinfelds, T. Ghosh, M. Prelas, R. V. Tompson and S. Loyalka, "The Research and Development of the Radioisotope Energy Conversion System," Trans. American Nuclear Soc., V84, American Nuclear Society, (ISSN: 0003-018X), pp 88-89 (2001). 34. M. Prelas, E. J. Charlson, E. M. Charlson, J. Meese, G. Popovici, and T. Stacy, "Diamond Photovoltaic Energy Conversion," Second International Conference on the Applications of Diamond Films and Related Materials, Editors M. Yoshikawa, M. Murakawa, Y. Tzeng and W. A. Yarbrough, MYU Tokyo, Pages 5-12 (1993).

4. A.B. Spitsyn, M.A. Prelas, Robert V. Tompson, T.K. Ghosh, "Impurity Removal from SIC using reversed Field Enhanced Diffusion with Optical Activation," Submitted to the Journal of Wide Band-Gap Materials. 
Acknowledgements: The authors gratefully acknowledge the support of this project by the DOE NEER program. 


\section{Summary of Project}

This project has involved a number of graduate students, most of which are on fellowships, and faculty. Dr. Alejandro Suarez (supported on fellowship) completed his Ph.D. in December 2001 based on his work with a diamond photovoltaic cell. Dr. Eric Stienfelds (supported on grant) completed his Ph.D. in the May 2003 based on his work in developing a model of the radioisotope energy conversion system, Dr. Dickerson Moreno (supported on grant) completed his Ph.D. in January of 2003 based on his work with phosphorous doping of diamond, Dr. Alexey Spitsyn (supported on fellowship) completed his Ph.D. in August of 2003 based on his work of making an Aluminum Nitride p-n junction, Mr. Bai-Ling Hsu completed his M.S. based his work in the doping AlN, Ms. Vanessa Velez (supported on fellowship) will complete her MS in the Fall of 2003 working on Kr and Ar excimers, and Mr. Angel Velez (supported on fellowship) has started working on this project working on diamond photovoltaic cells. The key findings of this work are the demonstration of $p-$ $\mathrm{n}$ junctions in diamond and aluminum nitride. The aluminum nitride $\mathrm{p}-\mathrm{n}$ junction is the first such junction developed and presents a very exciting technology for the production of not only electronic structures in aluminum nitride but also a potential VUV solid state diode. The other major achievement was 
the production of vacuum ultraviolet excimer emissions from krypton and argon at $147 \mathrm{~nm}$ and $129 \mathrm{~nm}$ using a microwave resonant cavity. This cavity design will allow for very efficient coupling of microwaves to plasma and the high efficiency production of VUV photons. Finally, our modeling study indicates that very high efficiency light weight radioisotope electric generators can be produced by scaling of this technology.

A conceptual remote power technology, which converts the energy emitted from radioisotopes to electrical energy without intermediate thermalization of the high-grade ion energy, is the topic of this proposal. The potential efficiency for this process, alone, is 40\%, nuclear energy to electrical energy. And, if combined with high-temperature thermionic conversion the nuclear to electrical energy conversion efficiency is $44 \%$ while the overall size of the system will remain small. These efficiencies compare to about $7.5 \%$ for the Radioisotope Thermionic Generator (RTG) system, used in deep space probes such as Cassini, in which nuclear energy is converted to electrical energy with intermediate thermalization. The key to the process is to first convert the high-grade ion energy to photon energy, which can then be directly converted to electrical energy. We term this process Photon-Intermediate 
Direct Energy Conversion (PIDEC). PIDEC is usable with radioisotopes, ion-producing plasma (hot) fusion reactions, as well as fission. In addition to improved efficiency, the PIDEC process also promises advantages in volume, mass, and cost. For application of the PIDEC process with radioisotopes, the radioisotope can be gaseous or solid. Solid radioisotope material can take the form of an aerosol of radioisotope microspheres or thin films with scale lengths significantly shorter than the range of the alpha or beta particles, dispersed in a fluorescer gas. In the first step of the process, the nuclear energy is transported to the fluorescer gas, which converts it into photons. Then, in the second step of the process, the photons are transported out of the reactor core to high band-gap photovoltaics, which efficiently convert the photon energy to electricity. We term this new mobile power concept, based on the PIDEC process, the Radioisotope Energy Conversion System (RECS).

Efficient production of excimer (non self-absorbing) fluorescers by ions has been demonstrated, but wide band-gap photovoltaics are not common at this time. However, several materials - such as SiC, C (diamond), and AlN - with band-gaps that match the energy of the photons emitted by excimer fluorescers do exist. We propose a study to address the bulk of the basic issues in 
the RECS in a three-year study.

The Photon-Intermediate Direct Energy Conversion (PIDEC) process, Figure 1, was first conceived for advanced-fuel high temperature (plasma) fusion [1, 2], which, unlike conventional D-T high temperature fusion, releases its energy as ions, rather than as neutrons. Advanced-fuel high temperature fusion, however, will not be available in the foreseeable future. And, at the time of preparation of this proposal, cold (solid-state) fusion is still too new to assess its feasibility. A more near-term potential source of ions became available with the development of the nuclear-driven fluorescer concept for nuclear-pumped lasers [3]. This concept makes feasible efficient ion-driven photon sources by removing the nuclear source material from the solid, where it prevented transmission of the photons, and moving it to the volume of the fluorescer gas by one of four methods; a gaseous radioisotope, radioisotopes embedded in thin films or as a radioisotope aerosol. The scale lengths of the thin film, fibers or aerosol are chosen small enough so that most of the nuclear energy escapes into the fluorescer gas. The PIDEC concept and the nuclear-driven fluorescer concept were combined to produce the Radioisotope Energy Conversion System (RECS) [4], Figure 3. 
RECS utilizes a two-step method for directly converting the energy of the charged particles from nuclear reactions into a usable energy form such as electricity, chemicals, or coherent light. (This basic energy conversion process is usable for any ion producing nuclear reaction.) In the first step the ions produced by nuclear reactions transfer their energy to an intermediate photon generator - a fluorescer medium. This portion of the RECS comprises what is called the nuclear-driven fluorescer or NDF (a medium which produces incoherent narrow-band electromagnetic radiation). In the second step, the intermediate photons from the nuclear-driven fluorescer are absorbed by a material, which converts the photons into a useful high-grade energy form. This portion of the RECS is the photon energy converter.

The RECS ion source comes from the decay of radioisotopes dispersed within a fluorescer gas. Effective dispersal is essential so that the ions produced by the isotope decay deposit most of their kinetic energy in the excimer gas rather than in the radioisotope material. There are at least four methods of achieving the desired dispersal: gaseous radioisotopes, radioisotopes embedded in thin films, or microscopic aerosol of radioisotopes. The efficiency of transport of the ion energy from the radioisotope to the fluorescer medium varies with the 
scale length of the thin film or aerosol, the chemical form of the radioisotope, and the uniformity of the radioisotope density. The variation of ion energy transport efficiency from a microsphere, to the fluorescer medium, with thin films and microspheres are discussed in Reference 6. Energy transport efficiencies are about 50\% for reasonably designed thin films and $70 \%$ for reasonably designed microspheres. The average atomic density in the medium must be on the order of $1 \times 10^{19}$ particles $\mathrm{cm}^{-}$ 3, enough to achieve reasonable power densities but not so great as to significantly degrade the transport of the fluorescence through the aerosol. Combining the constraints of efficiency, and optical transparency determines scale length of the thin film, fibers, or microspheres and number density. For example, a microsphere diameter of 5 ?m and number density of $1 \times 10^{6} \mathrm{~cm}^{-3}$, which should not create significant absorption of the fluorescence $[7,8]$, results in a fuel density of $0.63 \mathrm{mg} \mathrm{cm}^{-3}$, quite reasonable dimensions, and good number densities $\left(3.9 \times 10^{19}\right.$ atoms $\mathrm{cm}^{-3}$ ). An alternative, which couples $100 \%$ of the ion energy to the fluorescer medium, is a gaseous radioisotope such as Kr85 .

Excimer fluorescers are the most efficient radiators known and, because of their unbound lower levels, do not self absorb. They radiate in the single, relatively narrow, band of 
wavelengths required for efficient photovoltaic energy

conversion. The intrinsic fluorescence efficiencies of rare-

gas and rare-gas halide excimers, based on standard W-value

theory, are listed in column 1 of Table 1. Achievable

efficiencies should be near the intrinsic values at the power

and electron densities characteristic of nuclear reactions.

Table 1. Theoretical Maximum Intrinsic Photovoltaic, $\eta_{p v}$, and Ion-to-Electric, $\eta_{i e}$, Efficiencies for Selected RareGas and Rare-Gas Halide Excimer Fluorescers with Matched Wide-Band-gap Photovoltaic Materials.

\begin{tabular}{|l|l|l|l|l|l|l|}
\hline Excimer & $\eta_{\mathrm{f}}$ & $\mathrm{E}_{\lambda}(\mathrm{eV})$ & $\begin{array}{l}\text { Photovoltaic } \\
\text { Material }\end{array}$ & $\begin{array}{l}\text { Band-gap } \\
\text { Energy (eV) }\end{array}$ & $\eta_{\mathrm{pv}}=\mathrm{E}_{\mathrm{g}} / \mathrm{E}_{\lambda}$ & $\eta_{\mathrm{ie}}=\eta_{\mathrm{pv}} \eta_{\mathrm{f}}$ \\
\hline $\mathrm{Ar}_{2}{ }^{*}$ & 0.5 & 9.6 & AlN & 6.2 & 0.645 & 0.324 \\
\hline $\mathrm{Kr}_{2}{ }^{*}$ & 0.47 & 8.4 & AlN & 6.2 & 0.789 & 0.345 \\
\hline & 0.47 & 8.4 & Diamond & 5.5 & 0.655 & 0.308 \\
\hline $\mathrm{Xe}_{2}{ }^{*}$ & 0.48 & 7.2 & AlN & 6.2 & 0.861 & 0.413 \\
\hline & 0.48 & 7.2 & Diamond & 5.5 & 0.764 & 0.367 \\
\hline $\mathrm{ArF}^{*}$ & 0.35 & 6.4 & AlN & 6.2 & 0.969 & 0.339 \\
\hline & 0.35 & 6.4 & Diamond & 5.5 & 0.859 & 0.301 \\
\hline $\mathrm{KrBr}^{*}$ & 0.33 & 6 & Diamond & 5.5 & 0.917 & 0.302 \\
\hline $\mathrm{KrCl}^{*}$ & 0.31 & 5.6 & Diamond & 5.5 & 0.982 & 0.304 \\
\hline $\mathrm{Na}_{2}{ }^{*}$ & 0.46 & 2.84 & ZnSe & 2.7 & 0.951 & 0.437 \\
\hline & 0.46 & 2.84 & SiC & 2.4 & 0.845 & 0.389 \\
\hline $\mathrm{Li}_{2}{ }^{*}$ & 0.42 & 2.7 & CuAlSe & 2.6 & 0.963 & 0.404 \\
\hline & 0.42 & 2.7 & SiC & 2.4 & 0.889 & 0.373 \\
\hline
\end{tabular}

$\mathrm{E}_{\lambda}(\mathrm{eV})$ is the average photon energy $\mathrm{E}_{\mathrm{g}}$ is band-gap energy of the photovoltaic material

In fact one group has reported measuring nuclear-driven rare-gas excimer fluorescence efficiency higher than that predicted by W-value theory [9]. Measurements of actual fluorescence efficiencies at various laboratories, including Lawrence Livermore National Laboratory, have demonstrated high fluorescence efficiencies for excimers. Experiments with a variety of excitation sources (e.g. electrons, fission 
fragments, protons) and particle densities have given fluorescence efficiency values ranging from a few percent to as high as 68\% (see review paper by Prelas, et al. [10]). The most efficient excimer fluorescers are the rare-gas excimers. Nuclear-Driven Fluorescers are discussed in much greater detail in the paper "Nuclear-Driven Flashlamps" [10].

The key to the feasibility of the RECS is the photovoltaic Photon Energy Converter. The common impression of photovoltaics is that they cannot be very efficient. This misunderstanding comes from the fact that photovoltaics are most commonly employed as "solar cells." And solar cells are not very efficient, ranging from 10-20\% for commercial units and reaching as high as about 25\% for laboratory cells. However the low efficiency is more due to the characteristics of the solar spectrum than to the photovoltaics devices themselves, especially for the laboratory units with efficiencies of $\sim 25 \%$. The problem with the solar spectrum is that it is very broadband - its ratio of the average photon energy to the width (FWHM) of the spectrum ( $\left.E_{\text {mean }} / \Delta \mathrm{E}\right)$ is about 1. This is good for color vision but quite bad for efficient energy conversion. For excimers, however, this ratio is greater than 10. Under these conditions photovoltaics have intrinsic efficiencies of 75-95\%. 
Photovoltaic cells for use in photon-intermediate direct energy conversion of electricity will require the development of a doped semiconductor material with a band-gap that matches the UV radiation. With such photovoltaic cells, a system efficiency of 56\% for fusion ion driven fluorescence has been projected [2, 11]. Studies of fission ion driven fluorescence indicate that system efficiencies of about $40 \%$ are possible $[4,10]$. 


\section{Accomplishments Under Each Objective}

1) Characterization of excimer fluorescence sources (e.g., $\mathrm{Kr} 2 *-8.4 \mathrm{eV}$ photon).

1a) Build an excimer fluorescence source powered by microwaves for spectroscopy. A cell will be designed which can test VUV-Visible excimers (Year 1 Objective, Duration8 months).

An excimer fluorescence lamp was constructed using a microwave power source. A cylindrical microwave resonance cavity was designed for a $2.45 \mathrm{GHz}$ microwave source (See Figure 1). The cylindrical cavity can be designed to accommodate a fluorescence tube in which the gas flow can be varied (See Figure2).

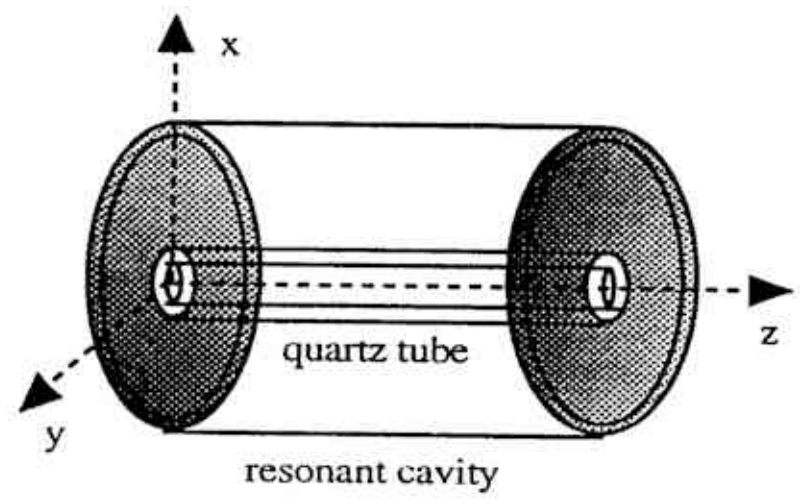

Figure 1. Cylindrical microwave resonant cavity.

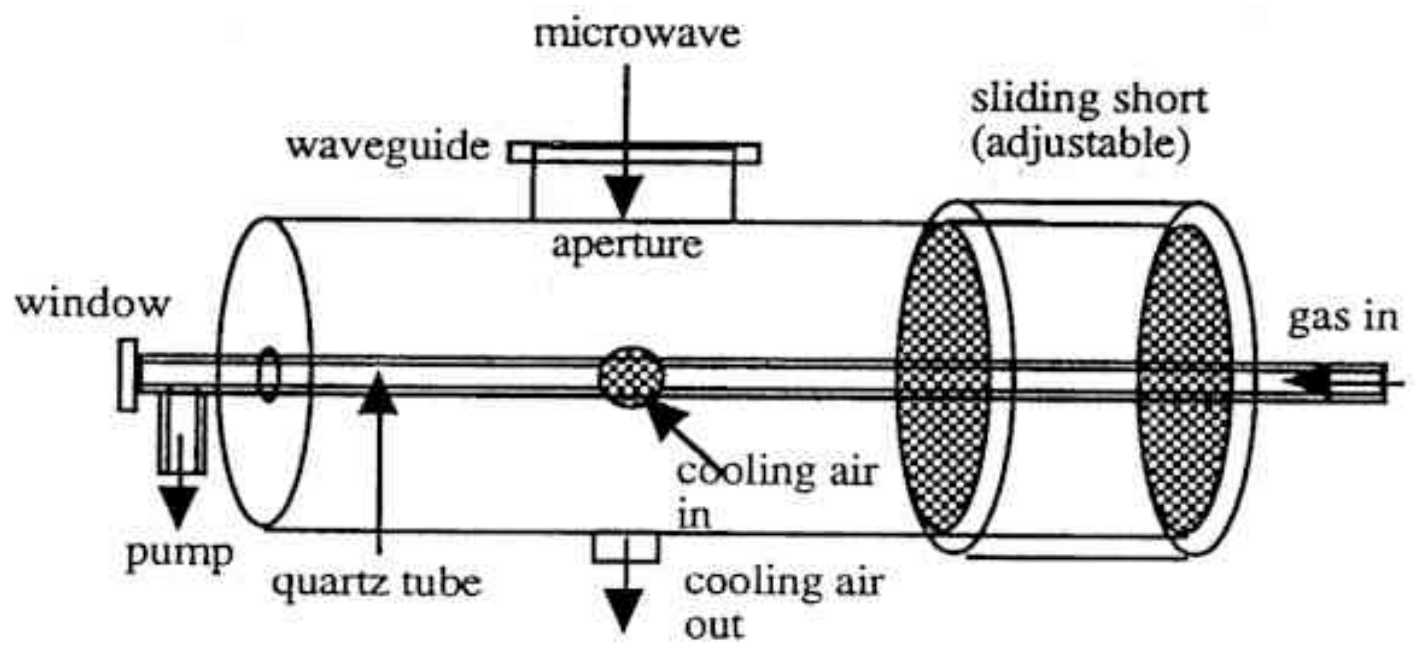


Figure 2. Air-cooled microwave resonant cavity for excimer emission.

The cavity window is made from calcium fluoride. Calcium fluoride is capable of transmitting wavelengths above 130 nanometers. This wavelength is in the vacuum ultraviolet, which means that the light is absorbed strongly in air. The light path from the window to the filtering and detection system must be either an inert gas such as helium or a vacuum. The widow is sealed to the input aperture of an Acton vacuum ultraviolet spectrometer. A vacuum ultraviolet multialkali photomultiplyer tube is used for the detector. It has a fused silica window that is capable of transmitting wavelengths above 150 nanometers. The setup is shown in Figure 3.

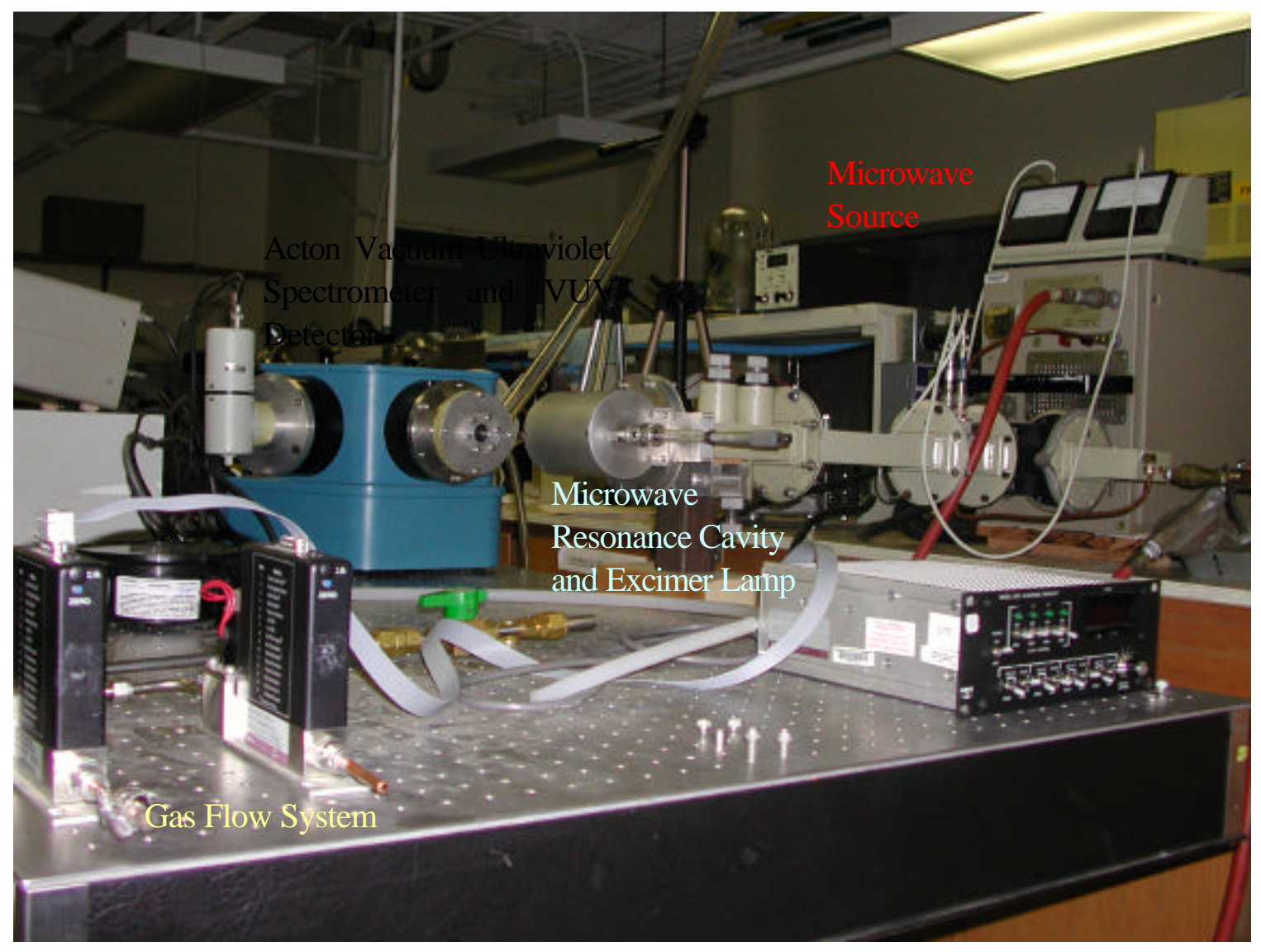

Figure 3. Microwave driven excimer lamp and vacuum ultraviolet spectrometer. 
The system was tested and operation was demonstrated as discussed next.

\section{1.b) Absolutely calibrate spectra from the excimer fluorescers (Year 1 Objective, Duration- 3 months).}

To calibrate the microwave driven excimer lamp, the cavity efficiency was determined for a variety of rare gases. The optimum length of the cavity was found for each gas mixture (as shown in Figure 4).

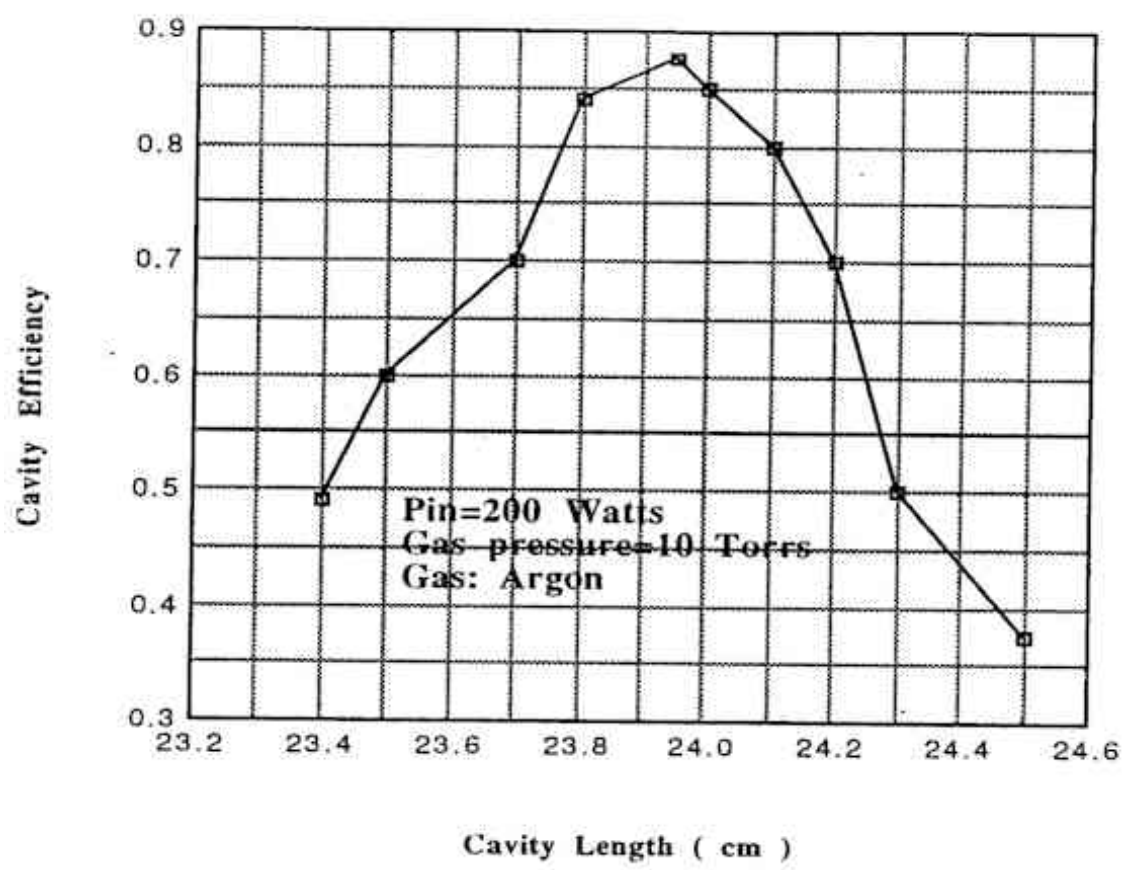

Figure 4. Optimizing length of the cavity for Argon gas.

In Figure 4, it is shown that the optimum length of the cavity is $23.95 \mathrm{~cm}$. The Power absorption in an optimized cavity for a specific fill gas can then be measured based on the microwave power absorbed and the microwave power that is reflected using the power monitor(see Figure 5). The cavity can 
be tuned by using the sliding short and the stub tuners.

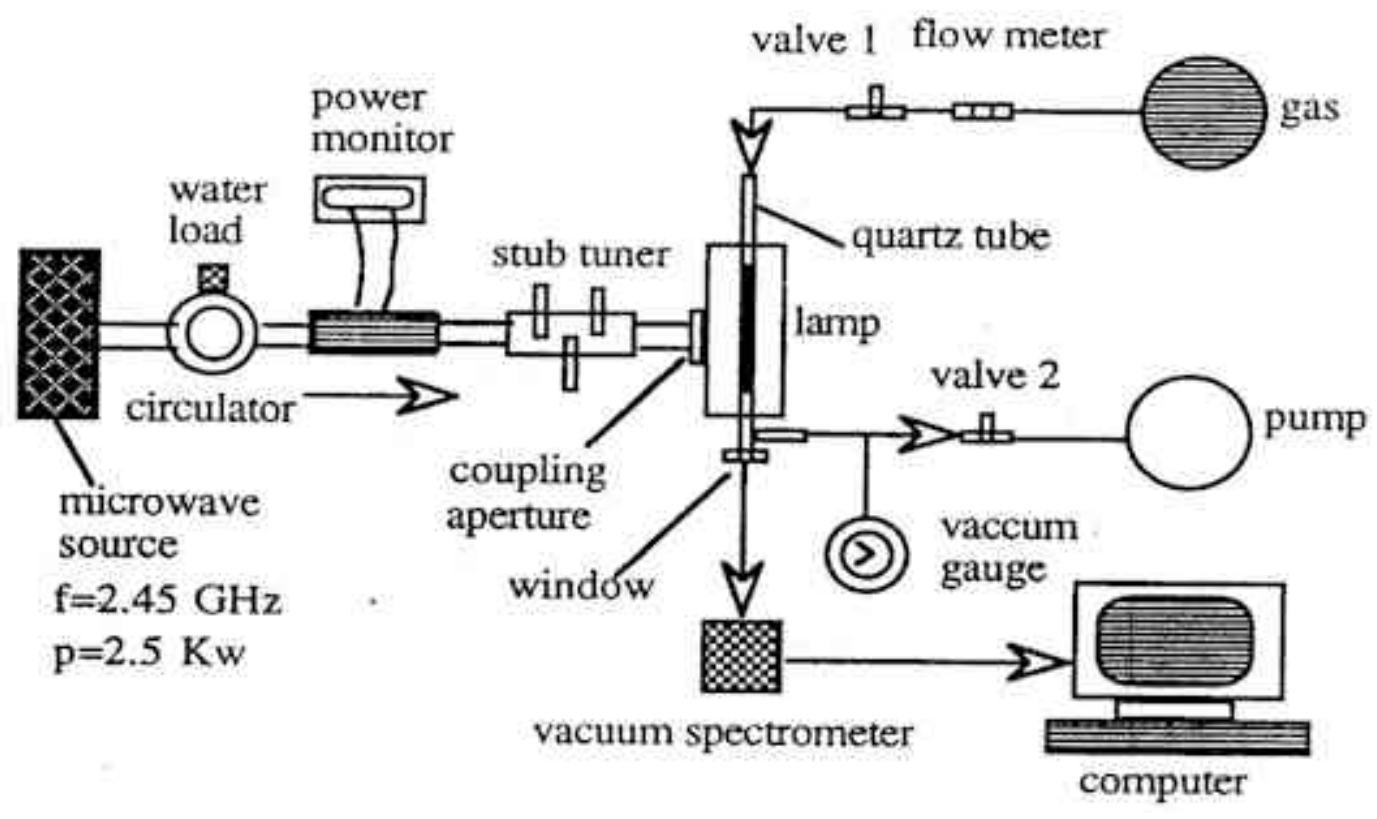

Figure 5. Microwave set up.

For argon, the emission was optimized and the excimer fluorescence at 129 nm was observed (See Figure 6).

\section{Data Set W Argon with Visible Plasma}

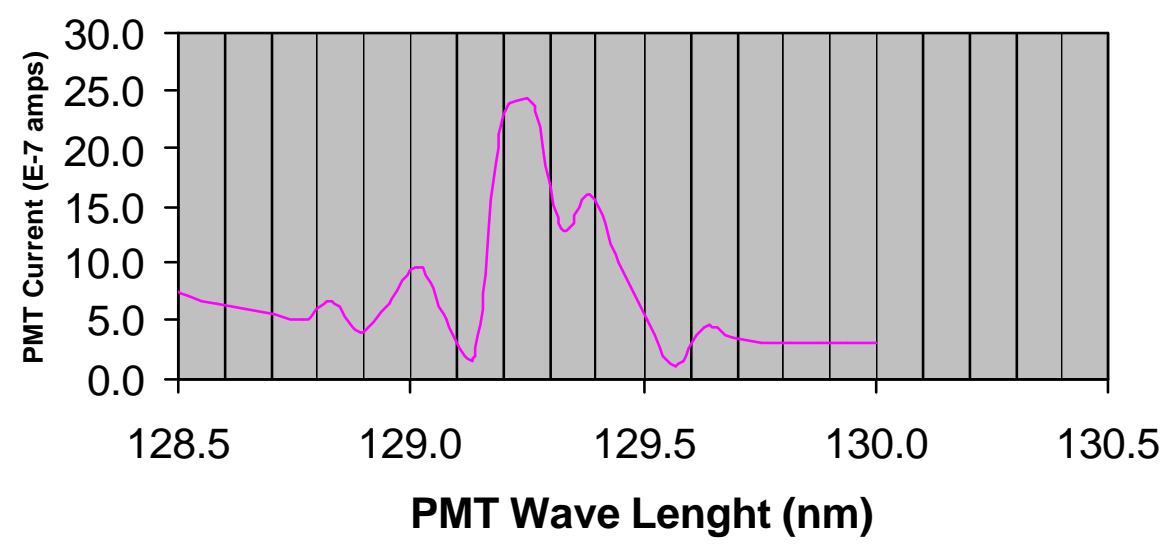


Figure 6. Excimer fluorescence spectrum from Ar.

The intensity of krypton excimer was also measured as shown in Figure 7 .

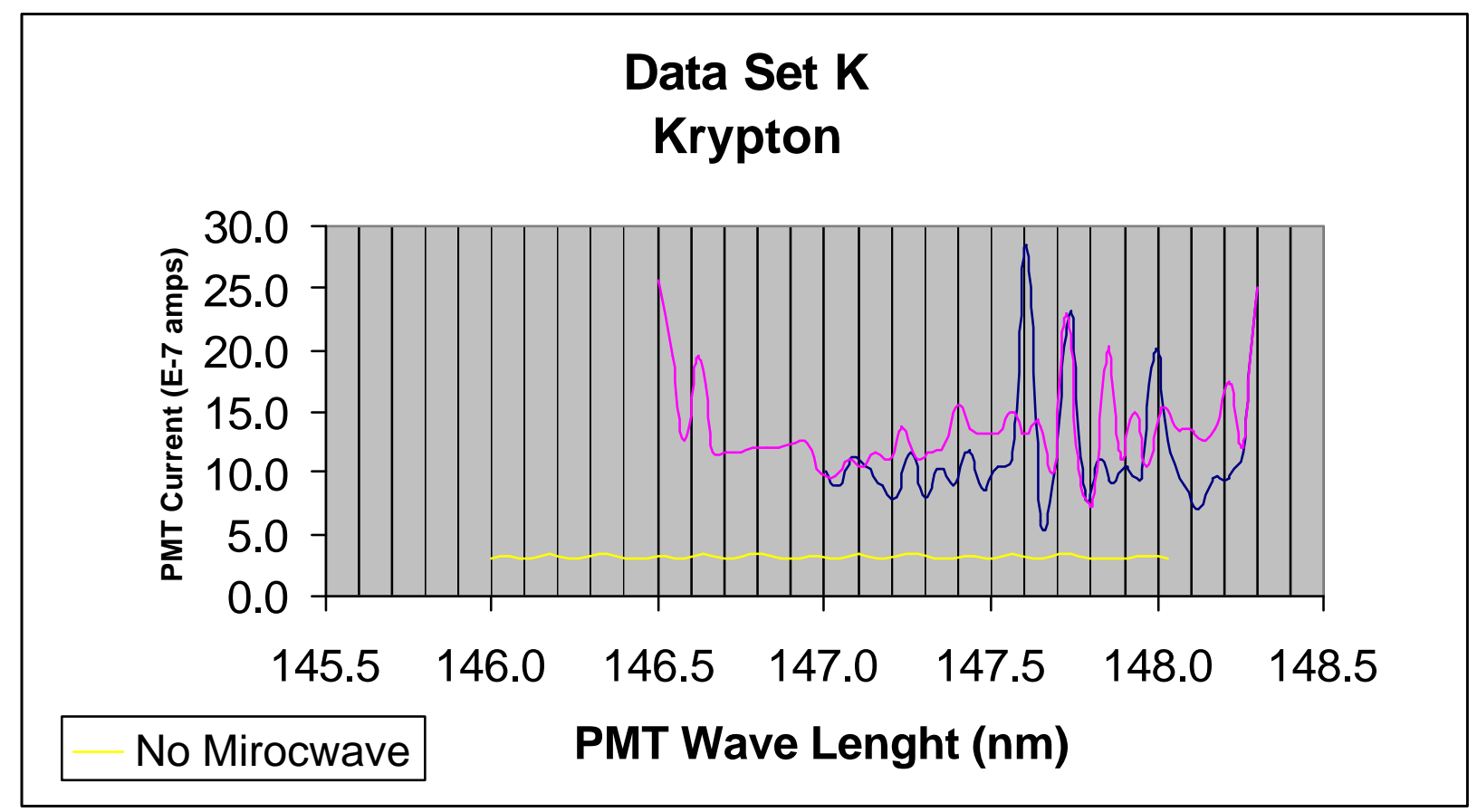

Figure 7. Kr vacuum ultraviolet spectra. Here the $147 \mathrm{~nm}$ excimer line is shown

The krypton excimer production efficiency can be calculated from this data by calculating the optical collection efficiency from the plasma, along its path through the spectrometer to the detector and the detector efficiency. 
1.c) Determine efficiencies for the production of excimer fluorescence (Year 1 Objective, Duration- 2 months).

The production efficiency can be calibrated with a source of a known wavelength or with a calibrated detector. We have a NIST VUV calibrated detector. The detector was purchased for a previous project. It must be recalibrated since its initial calibration is out of date. This objective could not be achieved because the calibration procedure was longer than originally estimated. We anticipate that the system will be calibrated early in the second year.

2) Develop photovoltaic cells that have band-gaps, which match the bandwidths of the excimer photon sources (Diamond - $5.5 \mathrm{eV}$, AIN - $6.2 \mathrm{eV}$ ).

2.a) Obtain polycrystalline or monocrystalline diamond (Year 1 Objective, Duration- 1 month).

We have obtained monocrystalline and polycrystalline diamond plates from Harris Diamond and Moscow state University respectively. In addition we have obtained silicon carbide and gallium nitride wafers. The band-gap of silicon carbide is 2.8 $\mathrm{eV}$ and the band-gap of gallium nitride is $3.2 \mathrm{eV}$. P-n junctions in both silicon carbide and gallium nitride have been formed. These materials are used in the production of light emitting blue diodes. We have added silicon carbide and gallium nitride to our list of possible photovoltaic materials due to the 
existing industry.

2.b) Obtain polycrystalline or monocrystalline AIN (Year 2 Objective, Duration- 1 month).

We have identified a source of high quality monocrystalline AlN plates. The plates have been ordered and will be shipped early in the second year of the project.

\section{2.c) Form junctions in diamond (Year 2 Objective, Duration-12 months ).}

A $p-n$ junction would exhibit the characteristics of a cross over point with $p$ and n-type impurities in the crystal lattice (Figure 8).

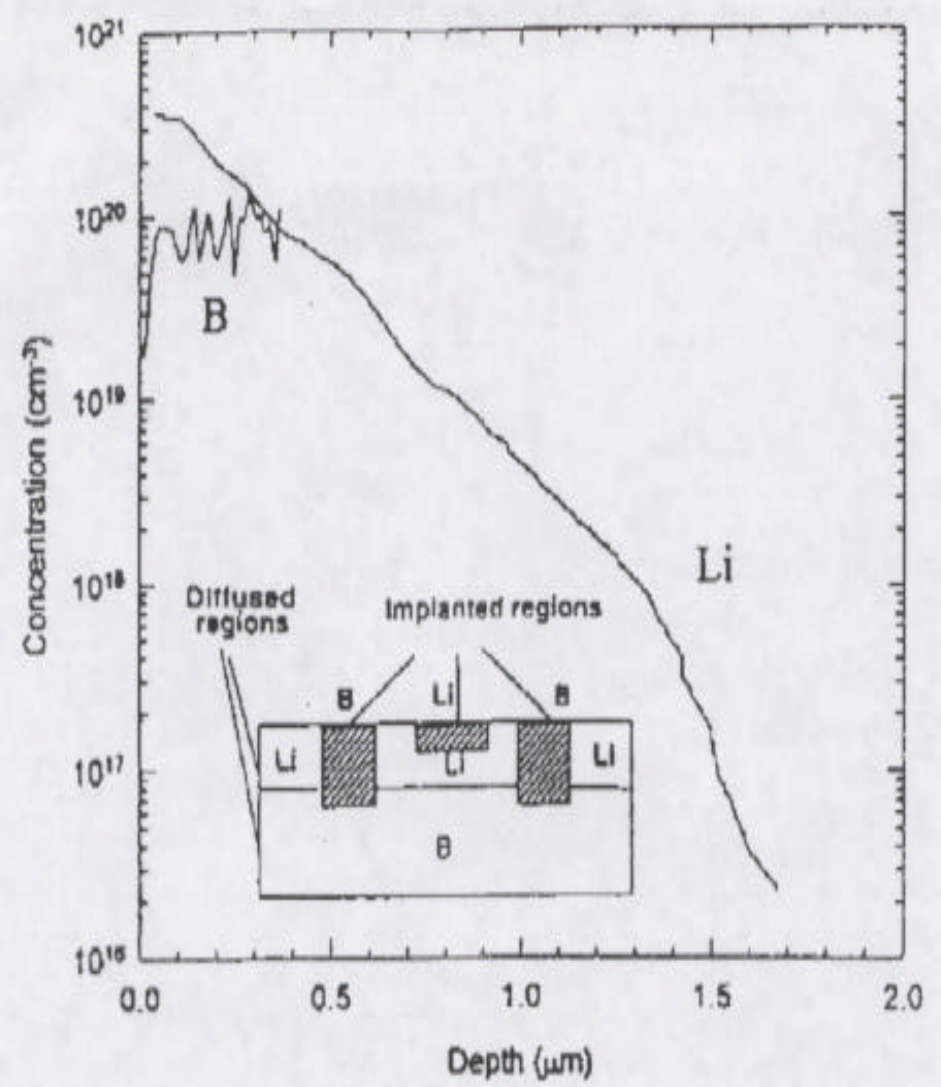

Figure 8. Overlap of p-type boron with n-type Li impurities in 
diamond.

Diamond plates were doped with boron (p-type) and sulfur (ntype) impurities. One of the interesting issues that arose was the uniformity of the impurity distribution. As can be seen in Figure 9, a boron doped diamond plate demonstrated a large amount of variation in conductivity, an indirect measure of boron density.

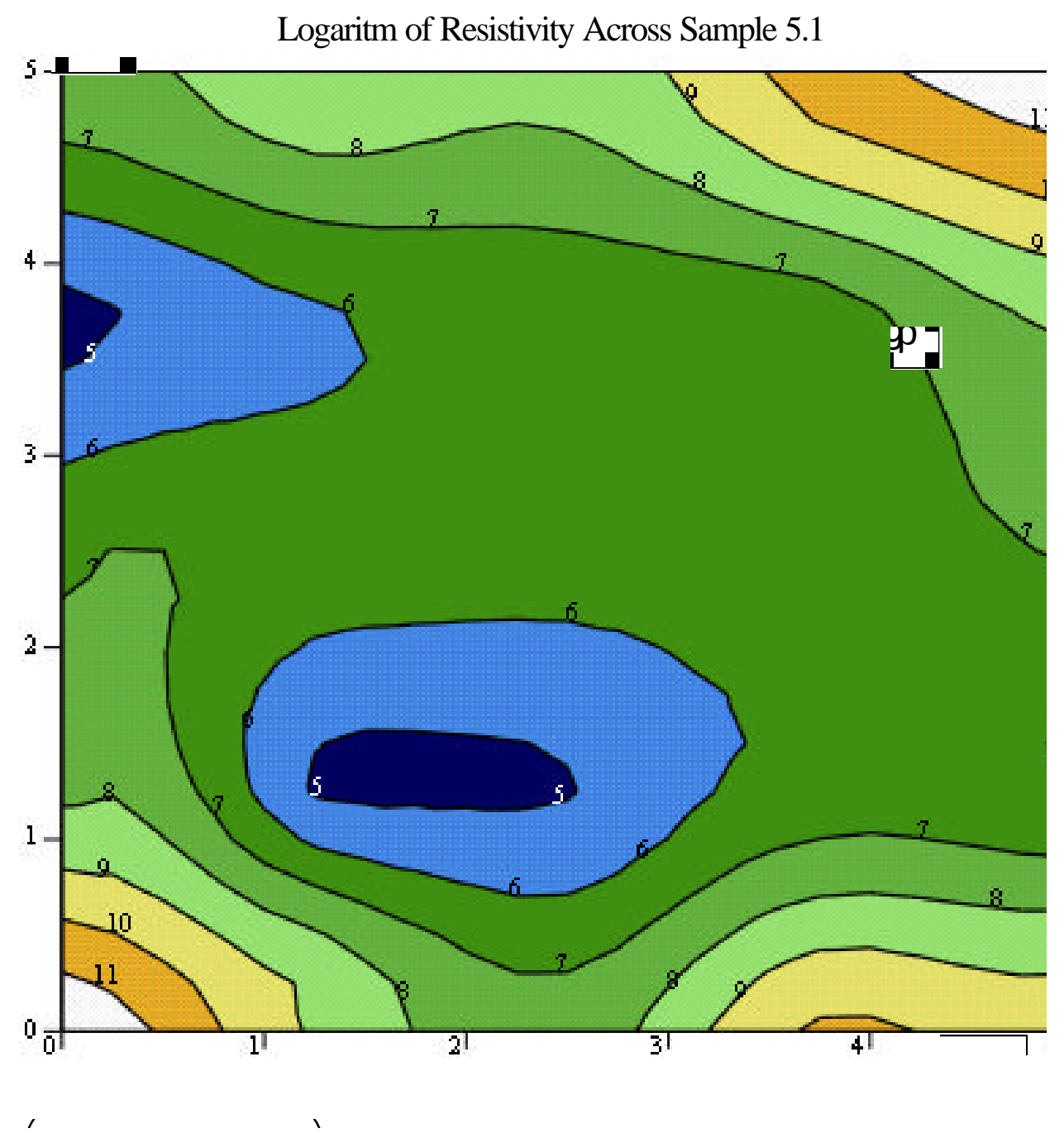

Figure 9. Two dimensional conductivity of a boron doped diamond 
plate.

The non uniform distribution was probably due to the distribution of boron powder impurity on the surface of the diamond during treatment.

Sulfur and phosphorous were used as n-type dopants. As described in appendix 1, sulfur and phosphorous were successfully added to a diamond crystal.

\section{2.d) Form junctions in AIN (Year 3 Objective, Duration- 12 months).}

As described in appendix 1, silicon carbide, gallium nitride and aluminum nitride were obtained. Both silicon carbide and gallium nitride are available as commercial p-n junctions. A key for high efficiency photovoltaics is to have as pure a crystal as feasible. Silicon carbide and gallium nitride have impurities that are added to the crystal during growth. The objective was to see if the silicon carbide and gallium nitride crystals could be purified. As described in appendix 1, 4-H and 6-H silicon were purified.

We also obtained aluminum nitride and to see if the crystals could be doped. We used $\mathrm{C}$ and $\mathrm{Si}$ as the n-type material and Ca and Mg as the p-type material. As described in appendix 1, we were able to incorporate large amounts of impurity into aluminum nitride crystals. One sample, in which both Mg and Si were used, did show promise. 


\section{2.e) Characterize surface of diamond (Year 1 Objective, Duration- 2 months).}

The surfaces of our diamond sample have been examined by
scanning electron microscope (SEM), Raman spectrum, current-
voltage (IV), and x-ray diffraction (see Figures 10 \& 11 for
example).

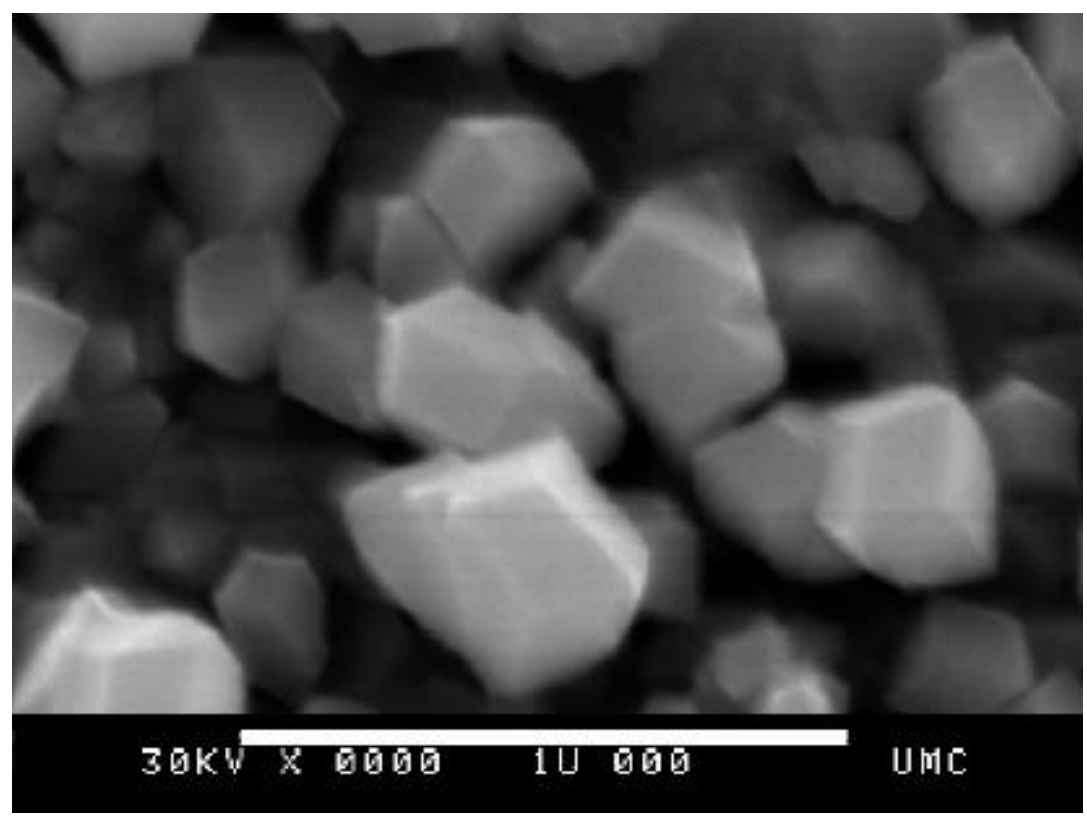

Figure 10. SEM of diamond films. 


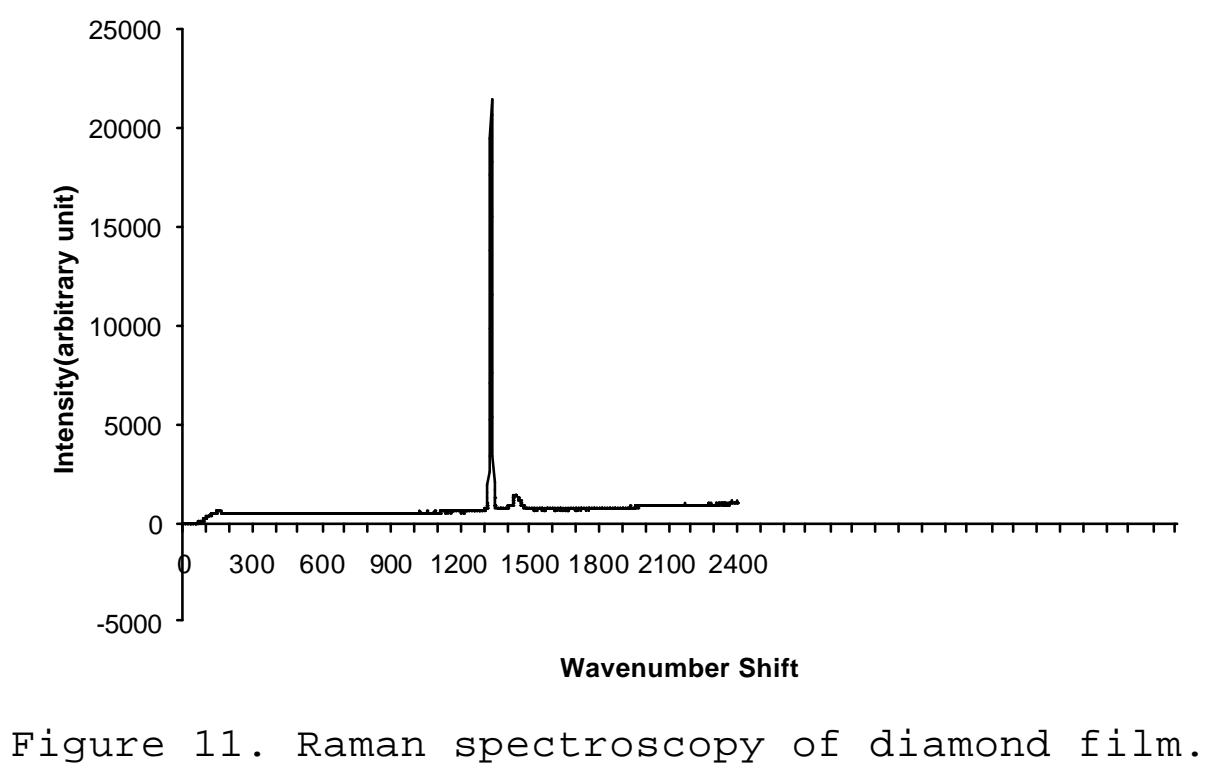

2.f) Characterize surface of AIN (Year 2 Objective, Duration- 2 months).

This step was accomplished as described in appendix 1.

2.g) Characterize the optical performance of the diamond photovoltaic cells (Year 2 Objective, Duration- 2 months).

This step is will be performed as part of year 3 as candidate impurities emerge.

2.h) Characterize the optical performance of the AIN photovoltaic cells (Year 3 Objective, Duration- 2 months).

$$
\text { Initial work with AlN films is also very promising. }
$$

With the samples we obtained of 10 to 40 micrometer thick AlN films grown on sapphire and silicon from the Institute of Physical Chemistry in Moscow Russia, we were able to make some key breakthroughs. The AlN film's carbon, silicon, calcium and 
magnesium contents of these films were below the detectable limit of the SIMS. We used FEDOA to dope samples of the film with carbon (graphite), silicon (purified powder), calcium (Ca powder) and magnesium ( $\mathrm{Mg}_{2} \mathrm{Si}$ powder). The samples were analyzed with SIMS and with I-V characteristics. In each case, the results demonstrated that a significant amount of impurity was added to film and that the film resistance had decreased. Two results are illustrative of this effort: 1) an n-type and p-type impurity can be co-doped into the crystal (see Figure 12) and 2) an AlN p-n junction (see Figure 13). Electroluminescence studies of the edge of the structure show distinct luminescence in the doped region (Figure 14).

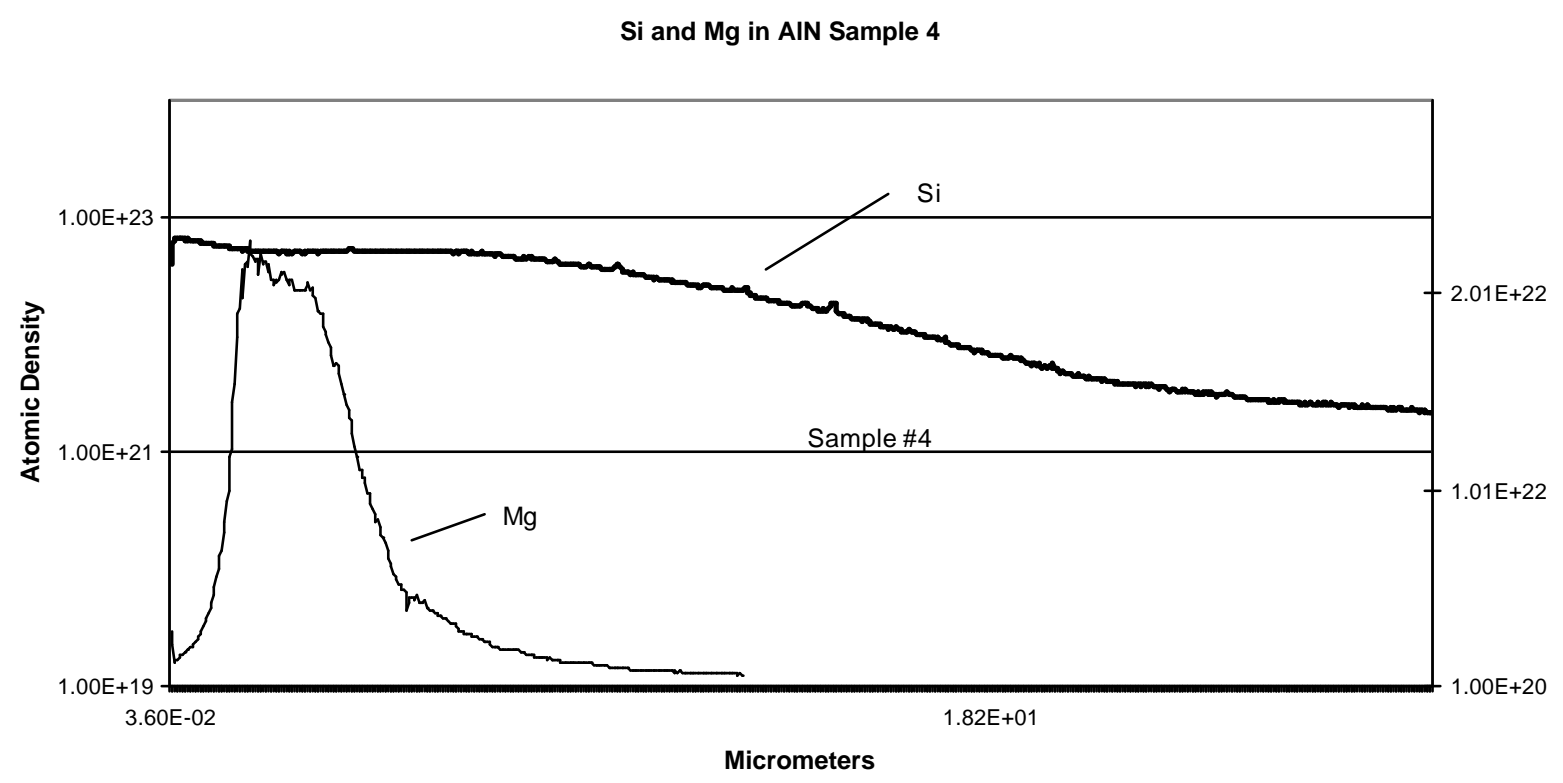


Figure 12. Shown is a SIMS analysis of AlN sample 4 which used $\mathrm{Mg}_{2} \mathrm{Si}$ as the impurity. It is clear that $\mathrm{Si}$ and $\mathrm{Mg}$ entered the AlN lattice differently demonstrating the possibility of forming junctions.

Our preliminary results indicate that FEDOA is a viable method of doping AlN. It is proposed to extend this research with the goal of producing a $\mathrm{p}-\mathrm{n}$ juction in $\mathrm{AlN}$ for the purpose of developing a ultraviolet photodiode. We also propose to study FEDOA as a means of removing impurities from AlN films. Also we would like to further explore the mechanisms of such removal process. This potential method of impurity removal might have commercial applications for many other crystals including SiC and GaN. 

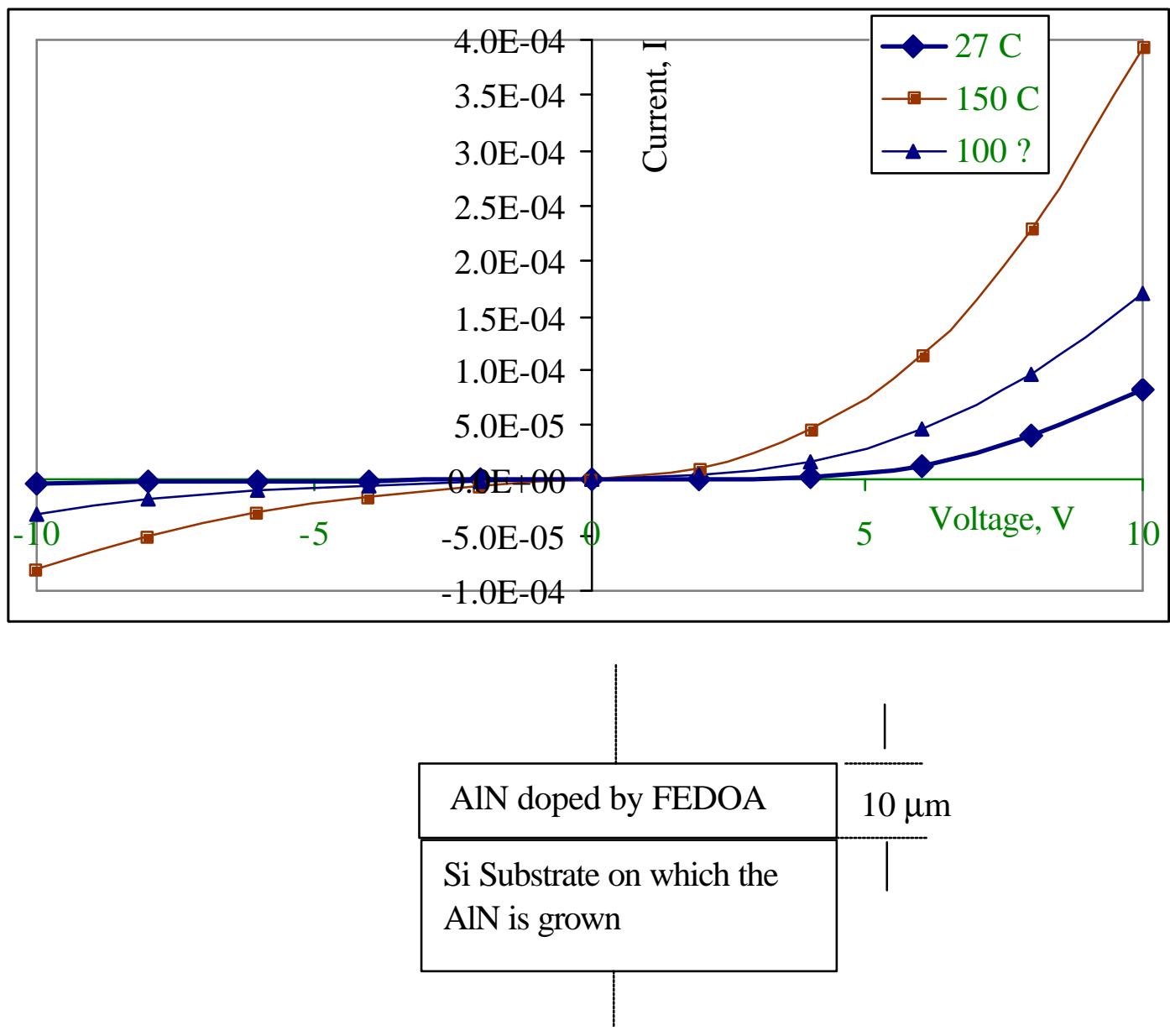

Figure 13. AlN p-n junction that demonstrated classical I-V characteristic of diode (journal article on this device under preparation). 


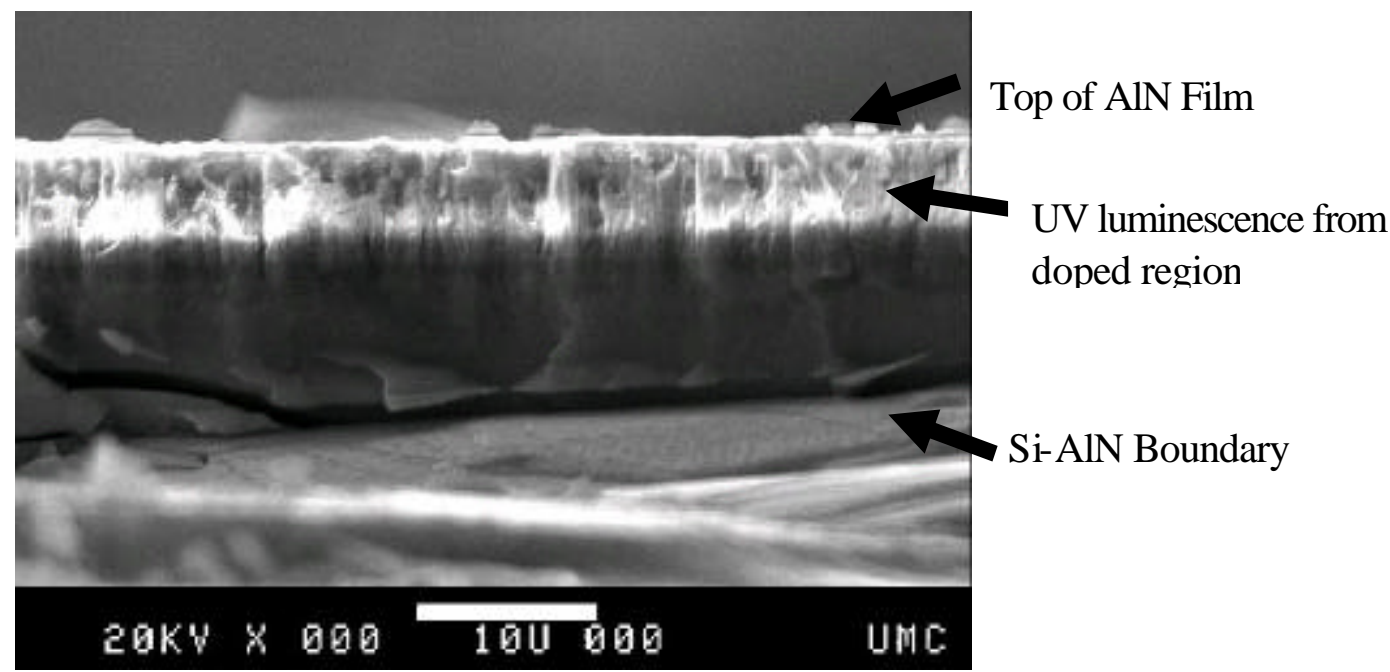

Figure 14. Edge UV luminescence from doped AlN film.

2.i) Develop antireflection coatings cells (Year 3 Objective, Duration- 4 months).

This step was not required because of the high index of refraction of AlN.

3) Build single tests cell electrical generator using the excimer and the photovoltaic cell, which best matches its bandwidth (Year 1 Objective, Duration 3 months).

The basics of the test cell have been assembled as shown in figure 3 .

3.a) Design a system to model a radioisotope driven excimer lamp using a discharge lamp and optical coupling system (Year 3 Objective, Duration 1 month).

A model for design was developed as part of the

Ph.D. dissertation of Dr. Eric Steinfelds.

4) Develop models for the transport of ions, the transport of photons and the transport of ionizing radiation. This will guide the experimental aspects of the project as well as predict scaling of the portable power generators (Year 1 Objective, Duration- 6 months ---Year 2 Objective, Duration 6 months ---Year 3 Objective, Duration 6 months). 
Finding solutions to the standard system of transport equations is necessary for a full treatment of the transport analysis of components 1), component 2), component 3), the beta particles, and the participating photons. Such solutions are pursued and found in this project by computational means and occasionally by semi-analytical means. We are using a Monte Carlo scheme to model the general RECS system. We discuss below some semi-analytical methods that support this effort in simulations with easily reproduced insightful examples. Such examples are good to have for the sake of having a few benchmark targets for our Monte Carlo simulations to be compared against.

There are some choices of geometry for a RECS unit for which it is feasible to solve the system of transport equations in a semi-analytical manner. For example, if 1), 2), and 3) are sequentially concentric and all spherically symmetric, then the transport equations of the system can be approximated by diffusion equations and almost exactly solved. This example will be discussed further towards the end of this discussion of concepts and theory.

For any of our RECS design, the transport equation for the blue (or UV) photons are monoenergetic, with photon source terms include for inelastically scattering beta particles. In 
our model, an inellastically scattering beta particle will release a large integral number of monochromatic blue (or UV) photons. This integral number shall here be expressed as 'het', 2x'het', 3x'het', etc.. The promptness of the photons generated by the fluorescing atoms varies. In the steady state, this has little effect on the equations. The transport equation for the beta particles is divided in a refined, but approximate manner into a system of $\mathrm{N}$ transport equations which represent the relations for angular fluxes of the beta particles at any one of $\mathrm{N}$ different energy levels. A beta particle at the nth energy level must release het $\times\left(\mathrm{E}_{\beta}\right) /(\mathrm{N}-1)$, $2 \times$ het $\times\left(\mathrm{E}_{\beta}\right) /(\mathrm{N}-1)$, or (higher integer) $\times$ bet $\times\left(\mathrm{E}_{\beta}\right) /(\mathrm{N}-1)$ in terms of $\mathrm{kinetic}$ energy when undergoing an inelastic collision with one of the fluorescing atoms. $E_{\beta}$ is the average kinetic energy of a beta particle immediately after emission.

For example, let us consider a source consisting of Sr-90 and a choice for fluorescing gas of Krypton (forming $\mathrm{Kr}_{2}{ }^{*}$ ). The mean energy of a beta ${ }^{(-)}$from the $\mathrm{Sr}-90$ source is $1 / 3 \times 546,000 \mathrm{eV}$, or $182,000 \mathrm{eV}$. The energy of the UV photon emitted from the fluorescing krypton is $8.43 \mathrm{eV}$ [12]. If we set 'het' equal to 100 , then we need 216 different energy levels for the beta ${ }^{(-)} \mathrm{s}$, which trickle down in energy in a linear manner. If the kinetic energy lost per beta collision 
is $100 \times(8.43 \mathrm{eV}) /(216-1), 100$ monochromatic UV photons were produced within the lifetime of the unbound beta particle.

Now that a particular example has been given, let us consider the somewhat generalized transport equations that need to be solved for the betas and photons within the three significant components of the RECS. The over-simplifications made in equations (1), (2), and (3) is that each fresh beta particle has perhaps only a few $100 \mathrm{eV}$ in kinetic energy and that the number of photons emitted per inelastic beta collision is either 3 or 6 , either of which is a very minuscule number for the operation of this model. Nevertheless, with only a little bit of judgment, equations (1), (2), and (3) can be modified so as to make 'het' within the text equal to a large integer rather than 3 for the number of photons produced. Once the angular flux as a function of location is determined in equation group (1), it is fairly easy to solve equation (2) for the blue (or UV) photons. Below we see the group equations (1) for the beta ${ }^{(-)}$particles (or alphas) which are cascading downwards in energy.

(1) $\frac{d \Psi_{B 1}}{\mathrm{v} 1 d t}+\Omega \cdot \nabla \Psi_{\mathrm{B} 1}+\Sigma_{\mathrm{t} B 1} \Psi_{\mathrm{B} 1}=\Sigma_{\mathrm{s}(4,1)} \frac{1}{4 \pi} \int \Psi_{\mathrm{B} 4}\left(\mathrm{r}, \Omega^{\prime}\right) \mathrm{d} \Omega^{\prime}+$

$$
+\Sigma_{\mathrm{s}(7,1)} \frac{1}{4 \pi} \int \Psi_{\mathrm{B} 7}\left(\mathrm{r}, \Omega^{\prime}\right) \mathrm{d} \Omega^{\prime}+\Sigma_{\mathrm{s}(1,1)} \frac{1}{4 \pi} \int \Psi_{\mathrm{B} 1}\left(\mathrm{r}, \Omega^{\prime}\right) \mathrm{d} \Omega^{\prime}
$$




$$
\begin{aligned}
& \frac{d \Psi_{B 4}}{\mathrm{v} 4 d t}+\Omega \cdot \nabla \Psi_{\mathrm{B} 4} \\
& +\Sigma_{\mathrm{t} \mathrm{B} 4} \Psi_{\mathrm{B} 4}=\Sigma_{\mathrm{s}(7,4)} \frac{1}{4 \pi} \int \Psi_{\mathrm{B} 7}\left(\mathrm{r}, \Omega^{\prime}\right) \mathrm{d} \Omega^{\prime}+ \\
& +\Sigma_{\mathrm{s}(10,4)} \frac{1}{4 \pi} \int \Psi_{\mathrm{B} 10}\left(\mathrm{r}, \Omega^{\prime}\right) \mathrm{d} \Omega^{\prime}+\Sigma_{\mathrm{s}(4,4)} \frac{1}{4 \pi} \int \Psi_{\mathrm{B} 4}\left(\mathrm{r}, \Omega^{\prime}\right) \mathrm{d} \Omega^{\prime} \\
& \frac{d \Psi_{B 7}}{\mathrm{v} 7 d t}+\Omega \cdot \nabla \Psi_{\mathrm{B} 7}+\Sigma_{\mathrm{t} \mathrm{B} 7} \Psi_{\mathrm{B} 7}=\Sigma_{\mathrm{s}(10,7)} \frac{1}{4 \pi} \int \Psi_{\mathrm{B} 10}\left(\mathrm{r}, \Omega^{\prime}\right) \mathrm{d} \Omega^{\prime}+ \\
& +\Sigma_{\mathrm{s}(13,7)} \frac{1}{4 \pi} \int \Psi_{\mathrm{B} 13}\left(\mathrm{r}, \Omega^{\prime}\right) \mathrm{d} \Omega^{\prime}+\Sigma_{\mathrm{s}(7,7)} \frac{1}{4 \pi} \int \Psi_{\mathrm{B} 7}\left(\mathrm{r}, \Omega^{\prime}\right) \mathrm{d} \Omega^{\prime} \\
& \frac{d \Psi_{B M a x}}{\mathrm{v}_{(\text {Max })} d t}+\Omega \bullet \nabla \Psi_{\mathrm{BMax}}+\Sigma_{\mathrm{t} \text { BMax }} \Psi_{\mathrm{BMax}}=0 .
\end{aligned}
$$

Below we see the transport equation for the photons which are produced by the betas (or a`s) colliding inelastically with the fluorescing atoms via eximer formation of the atoms.

$$
\begin{aligned}
& \frac{d \Psi_{\gamma}}{\mathrm{v} \gamma d t}+\Omega \nabla \cdot \Psi \gamma+\Sigma_{\mathrm{t}(\gamma)} \Psi \gamma=\Sigma_{\mathrm{s}(\gamma)} \frac{1}{4 \pi} \int \Psi_{\gamma}\left(\mathrm{r}, \Omega^{\prime}\right) \mathrm{d} \Omega^{\prime}+ \\
& + \text { het } \cdot \Sigma_{\mathrm{s}(4,1)} \frac{1}{4 \pi} \int \Psi_{\mathrm{B} 4}\left(\mathrm{r}, \Omega^{\prime}\right) \mathrm{d} \Omega^{\prime}+\text { het } 2 \cdot \Sigma_{\mathrm{s}(7,1)} \frac{1}{4 \pi} \int \Psi_{\mathrm{B} 7}\left(\mathrm{r}, \Omega^{\prime}\right) \mathrm{d} \Omega^{\prime}+ \\
& + \text { het } \cdot \Sigma_{\mathrm{s}(7,4)} \frac{1}{4 \pi} \int \Psi_{\mathrm{B} 7}\left(\mathrm{r}, \Omega^{\prime}\right) \mathrm{d} \Omega^{\prime}+\text { het } 2 \cdot \Sigma_{\mathrm{s}(10,4)} \frac{1}{4 \pi} \int \Psi_{\mathrm{B} 10}\left(\mathrm{r}, \Omega^{\prime}\right) \mathrm{d} \Omega^{\prime}+ \\
& \quad \ldots \quad \ldots \\
& + \text { het } \Sigma_{\mathrm{s}(\text { Max }, \text { Max }-3)} \frac{1}{4 \pi} \int \Psi_{\mathrm{B}(\operatorname{Max})}\left(\mathrm{r}, \Omega^{\prime}\right) \mathrm{d} \Omega^{\prime} \\
& \quad \text { where het }=3 \text { and het } 2=6 .
\end{aligned}
$$

Within the region where the radioisotope material is present, an energetically modest calculational procedure is to use the approximate relations of the local flux values for the newly mobile beta ${ }^{(-)}$particles as seen in equations (3a) and (3b). 
(3a) $\frac{d \Psi_{B M a x}}{\mathrm{v}_{\text {(Max }} d t}+\Omega \nabla \cdot \Psi_{\text {BMax }}+\Sigma_{\mathrm{t} \text { BMax }} \Psi_{\text {BMax }}=0-\frac{1}{4 \pi} \frac{d \rho_{\text {radios }}}{d t} \quad((\Leftarrow$ Source term $))$

$$
\begin{aligned}
& \frac{d \Psi_{B(\text { Max }-3)}}{\mathrm{V}_{(\text {Max }-3)} d t}+\Omega \nabla \Psi_{\mathrm{B}(\operatorname{Max}-3)}+\Sigma_{\mathrm{t} \mathrm{B}(\mathrm{Max}-3)} \Psi_{\mathrm{B}(\mathrm{Max}-3)}= \\
& \Sigma_{\mathrm{s}(\mathrm{M}, \mathrm{M}-3)} \frac{1}{4 \pi} \int \Psi_{\mathrm{BMax}}\left(\mathrm{r}, \Omega^{\prime}\right) \mathrm{d} \Omega^{\prime} .
\end{aligned}
$$

Because of the small width of the radioisotope (which ought to be comparable to the mean free path hopefully), it is expected that only $\Psi_{\mathrm{B}(\mathrm{M}-3)}$ and $\Psi_{\mathrm{B}(\mathrm{M})}$ are significant within the region of the radioisotope. Analytically (and computationally) speaking, it is sufficient to first compute the functional values of $\Psi_{\mathrm{B}(\mathrm{Max}-3)}$ and $\Psi_{\mathrm{B}(\mathrm{Max})}$ at the boundary which separates the radioisotope from the fluorescing gas, within a mean free path. It is useful to use $\Psi_{\mathrm{B}(\mathrm{Max})}\left(\mathrm{r}_{\text {boundary }}, \Omega\right)$ and $\Psi_{\mathrm{B}(\mathrm{Max}-3)}\left(\mathrm{r}_{\text {boundary }}, \Omega\right)$ as sources on the effective boundary of the source to determine all of the $\Psi_{\mathrm{B}(\mathrm{i})}(r, \Omega)$ in terms of their functional relations to distance $r$. Group equations (1) apply to the region of the fluorescing gas. Briefly the symbols of equations (1), (2), and (3) will be explained. $\Psi_{\mathrm{B}(\mathrm{i})}$, or $\Psi_{\mathrm{Bi}}$ is the angular flux of beta particles of energy level i. $\Psi_{\gamma}$ is the angular flux of a blue or UV photon. The maximum energy level matches $\max$ of $B_{\operatorname{Max}}$. The beta particles have Maxtl energy levels. 'vi' is the speed of beta particles labeled as Bi. $\Psi_{\gamma}$ is the flux of blue or UV photons. 
' $v$ ' is light's speed. $\Sigma_{\mathrm{t} \text { Bi }}$ is a particular macroscopic crosssection for beta particles labeled as $\mathrm{Bi} . \Sigma_{\mathrm{s}(7,4)}$ is the macro cross-section for beta particles at level 7 which drop down to energy level 4.

We are implementing a Monte Carlo scheme to calculate the spatial distribution of both the beta as well as the photon fluxes. We need to store in memory the stochastically generated data of the locations and levels of energy of the inelastic beta collisions. Then the acting simulation program needs to go to the stochastic locations of inelastic collisions and (by applying a Monte Carlo activity generator) generate 'het' or $2 x^{\prime}$ 'het' or more monoenergetic photons. These generated photons need to be given direction in a random and preferably with isotropic bias. In the first code, every one of the final positions of the blue (UV) photons is stochastically generated and in turn recorded. Within component 2), any one location of a beta(-) collision is equivalent to the location of the transformed fluorescing atom or eximer which subsequently emits 'het' or $2 x^{\prime}$ 'het' or more photons. In order to avoid the time cost of individual processings, the newer computational simulation will most likely sample those fluorescing atoms which have been impacted by beta particles. Only a favored atom of a minor group sample 
will stochastically generate 'het' or $2 x^{\prime h e t '}$ photons. The other fluorescing atoms of the group sample will produce photons following paths exactly parallel to the photons produced by the excited favored atom of the sample. For a successful computer simulation of a desirable RECS unit, the vast majority of blue photons need to penetrate into component 3) rather than transforming into heat in component 2) or component 1) of the RECS.

The Monte Carlo code is still being improved. However, a few simple examples for RECS units have been considered. Although such a design is not most economical in terms of operation, the easiest system to consider is a RECS unit with complete spherical symmetry. Such a RECS can be solved almost precisely by analytical means if we take the liberty to employ "Fick's Law" :

$$
\begin{aligned}
& \vec{J}(\vec{r})_{\text {particles }}=-1 /(3 \Sigma) \cdot \vec{\nabla} \Phi(\vec{r})_{\text {particles }} \quad \text {, where } \\
& \Phi(\vec{r})_{\text {particles }}=\oint \Psi(\vec{r}, \Omega) d \Omega \quad .
\end{aligned}
$$

By using equation (4) and considerations of flux, equations (1), (2), and (3) can be approximated as multigroup diffusion equations. For a spherically symmetric RECS unit, the diffusion equations are conceptually easy to solve. The warning given is that the diffusion equations often do a very 
poor job explaining transport of particles across a boundary of different media. Thus the diffusion solutions found for the approximate representations of equations (1), (2), and (3) must be considered to be of merely limited accuracy. However, the solving the diffusive equations here proves that it is not difficult to model RECS units with spherically symmetric geometry or broad planar 'sandwich' layered design.

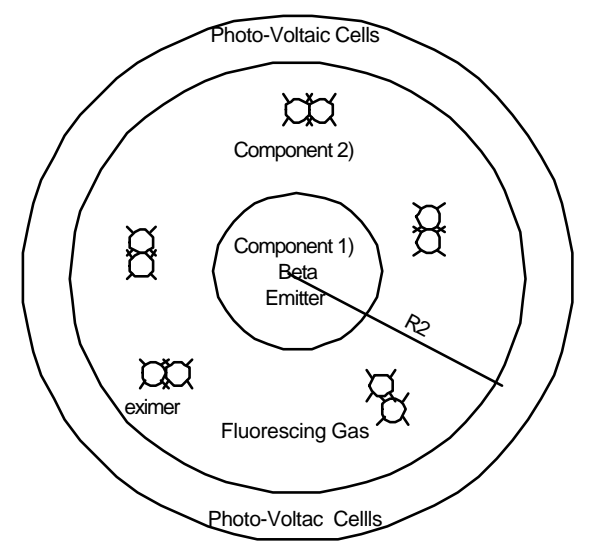

Figure 12. Illustration of a spherical RECS.

Beta particles travel mainly from component 1) (in the central sphere) into the fluorescing gas, which immediately surrounds component 1). The outer spherical shell consists of uniformly placed photo-voltaic cells with respect the shell surface.

A RECS with spherical symmetry is illustrated in Figure (12). For such a RECS as shown in Figure (10), we get solutions to the designated diffusion equations in the form 
written below. $\Phi_{\gamma}^{\text {Tran }}(r)$ is the flux of blue (or UV) photons in the outer region designated as the ensemble of photo-voltaic cells.

$$
\begin{aligned}
& \Phi_{\gamma}^{\text {Tran }}(r)=P_{\gamma} \cdot \frac{\exp \left(-K_{\gamma}^{\text {tran }} \cdot r\right)}{r} \quad \text {, where } \\
& K_{\gamma}^{\text {tran }}=\sqrt{3 \cdot \sum_{\gamma(a b)} \cdot \sum_{t(a b)}} \quad .
\end{aligned}
$$

The amplitude $\mathrm{P}_{\gamma}$ is linearly determined by the amplitude of the flux of the photons in the gaseous region, the amplitudes of flux of the betas, and ultimately by the magnitude of the radioactivity of the source. The flux of the blue (or UV) photons in the gaseous region is found by solving the diffusive representation of equation (2) (Eqn (2)), which accounts for photon sources from the fluorescing atomic eximers. Without going into painstaking detail, the form for the diffusive solution of the flux in the gaseous (fluorescing) component of this RECS is given next. 'G' stands for gas.

$$
\begin{aligned}
& \Phi_{\gamma}^{G}(r)=A_{\gamma}^{G} \cdot \frac{\exp \left(K_{\gamma}^{G} \cdot r\right)}{r}+B_{\gamma}^{G} \cdot \frac{\exp \left(-K_{\gamma}^{G} \cdot r\right)}{r}+\text { ext } \ldots \\
& \ldots .+\sum_{i=1}^{\operatorname{Im} a x} A_{\text {Beta }(\text { het } i+1)}^{G} \cdot \operatorname{transform}(\text { het } \cdot i+1) \cdot \frac{\exp \left(K_{\gamma}^{G} \cdot r\right)}{r}+\text { next } \ldots .
\end{aligned}
$$




$$
\ldots+\sum_{i=1}^{\operatorname{Im} a x} B_{\text {Beta }(\text { het } \cdot i+1)}^{G} \cdot \operatorname{transform}(\text { het } \cdot i+1) \cdot \frac{\exp \left(-K_{\gamma}^{G} \cdot r\right)}{r}
$$

Note that 'het'-Imax+1 equals $N$, which is the number of energy level divisions for the beta particle. In the region of the source, the diffusive solution is straight forward enough.

$$
\Phi_{\gamma}^{\text {source }}(r)=M_{\text {source }} \cdot \frac{\sinh \left(-K_{\gamma}^{\text {source }} \cdot r\right)}{r}
$$

In keeping with the purpose of a RECS, it is desired to have the flux for the blue (or UV) photons entering the radioactive source material to be as small as possible. In the case of the spherical symmetric RECS of Figure (12), we want P $\gamma$ of $\Phi_{\gamma}^{\text {Tran }}(r)$ of Eqn (5) to be as large as possible. Within the region of photovoltaic cells, the number of photons absorbed per unit time to make electricity can be evaluated by the integral:

$$
\begin{gathered}
\text { Rate }(\text { of_Photons_Absorbed }) \\
\text { by_photo-voltaic_cell }
\end{gathered} \approx \int_{R_{2}}^{R_{2}+2 \sum_{a b s}^{(\text {Tran })}} 4 \pi r r^{2} d r \cdot \Phi_{\gamma}^{\text {Tran }}(r) \cdot \sum_{\text {absorb }}
$$

The point of equations (5) through (8) is not their accuracy, but the fact that by almost analytical means it is possible to calculate the photon energy transferred to the photo-voltaic cells of component 3) from a beta emitting source. The Monte Carlo method for transport analysis of course works well for simple designs such as the spherically symmetric RECS of Figure (10). Moreover, the Monte Carlo method shows very 
strong promise for modeling the photon flux distribution in

more elaborate design the 'sandwiched' group of boxed

components for RECS.

References:

1. G. H. Miley, Direct Conversion of Nuclear Radiation Energy, American Nuclear Society, Hinsdale (Ill.) 1970.

2. M. A. Prelas, "A Potential UV Fusion Light bulb for Energy Conversion", Bult. of the Am. Phys. Soc., 26(1), 1045, 1981.

3. M. A. Prelas, and F. P. Boody, "Charged Particle Transport in Uranium Micropellets," 1982IEEE Int. Conf. on Plasma Sci., IEEE Publication Number 82CH1770-7, 1982; also Boody, F.P., and Prelas, M.A., "Photolytic Dual-Media Nuclear Pumping of Excimer Lasers", Excimer Lasers$\underline{83}$, C. K. Rhodes, H. Egger, and H. Plummer, editors, AIP, NY, 379-386 (1983).

4. M. A. Prelas, F. P. Boody, M. Zediker and M. Rowe, "A Direct Energy Conversion Technique Based on an Aerosol Core Reactor Concept", 1984 IEEE Int. Conf. on Plasma Sci., IEEE Publication Number: 84CH1958-8, 38, 1984.

5. E Lewis, Nuclear Science and Engineering, 25, 359 (1966)

6. A. Chung, and M. A. Prelas, "Charged Particle Spectra From ${ }^{10} \mathrm{~B}$ and $\mathrm{UO}_{2} \mathrm{Slab}$ and Spherical Sources," 1983 IEEE Int. Conf. on Plasma Sci., IEEE Publication Number 83CH1847-3, 1983; also A. K. Chung and M. A. Prelas, "Charged Particle Spectra From U235 and B-10 Micropellets and Slab Coatings," Laser and Particle Beams, 2(2), 201-211 (1984).

7. F. P. Boody, M. A. Prelas, and M. S. Zediker, "An Aerosol Core Reactor/Laser for Space Based Use," 1st Symposium on Space Nuclear Power Systems, Albuquerque, NM, Jan. 1984.; also M. A. Prelas, F. P. Boody, and M.S. Zediker, "An Aerosol Core Nuclear Reactor for Space-Based High Energy/Power Nuclear-Pumped Lasers," Space Nuclear Power Systems, Vol. IV, M. S. ElGenk and M. D. Hoover, editors, Orbit Book Co., 267 (1986).

8. G. Gu, "Analysis of High Power/Energy Nuclear-Pumped Laser/Reactor Concepts", Ph.D. Dissertation, Nuclear Engineering Program, University of Missouri-Columbia, Dec. 1987.; also G. $\mathrm{Gu}$, M. A. Prelas, and J. F. Kunze, "Neutronic Considerations of Nuclear-Pumped Laser/Reactor Concepts", Space Nuclear Power Systems, M. S. El-Genk and M. Hoover Editors, Orbit Book, Co. Malbar Fl, 155-160 (1988).

9. R. A. Walters, J. D. Cox, and R. T. Schneider, Trans. Am. Nuc. Soc., 34 p.810, 1980.

10. M. A. Prelas, F. P. Boody, G. H. Miley, and J. Kunze, "Nuclear-Driven Flashlamps", Lasers and Particle Beams, 6(1), 25, 1988; also, M. A. Prelas, and S. Loyalka, Progress in Nuclear Energy, 8, 35-52, 1981 
11. M. A. Prelas, J. Romero, and E. Pearson, "A Critical Review of Fusion Systems for Radiolytic Conversion of Inorganics to Gaseous Fuels", Nuclear Technology Fusion, 2(2), 143, 1982.; also M. A. Prelas, F. P. Boody, M. Zediker and M. Rowe, "A Direct Energy Conversion Technique Based on an Aerosol Core Reactor Concept", 1984 IEEE Int. Conf. on Plasma Sci., IEEE Publication Number: 84CH1958-8, 38, 1984.

12. M.A. Prelas, F.P. Boody, D.J. Charlson, G.H. Miley; Progress in Nuclear Energy; Vol. 23 No 3; pages 223-240; Pergamon Press, plc.; 1991. Printed 1t in Great Britain-all r. res.- in 1990. 


\section{Appendix 1}

Slide presentation from the June 2002 ANS meeting. 
Slide 1

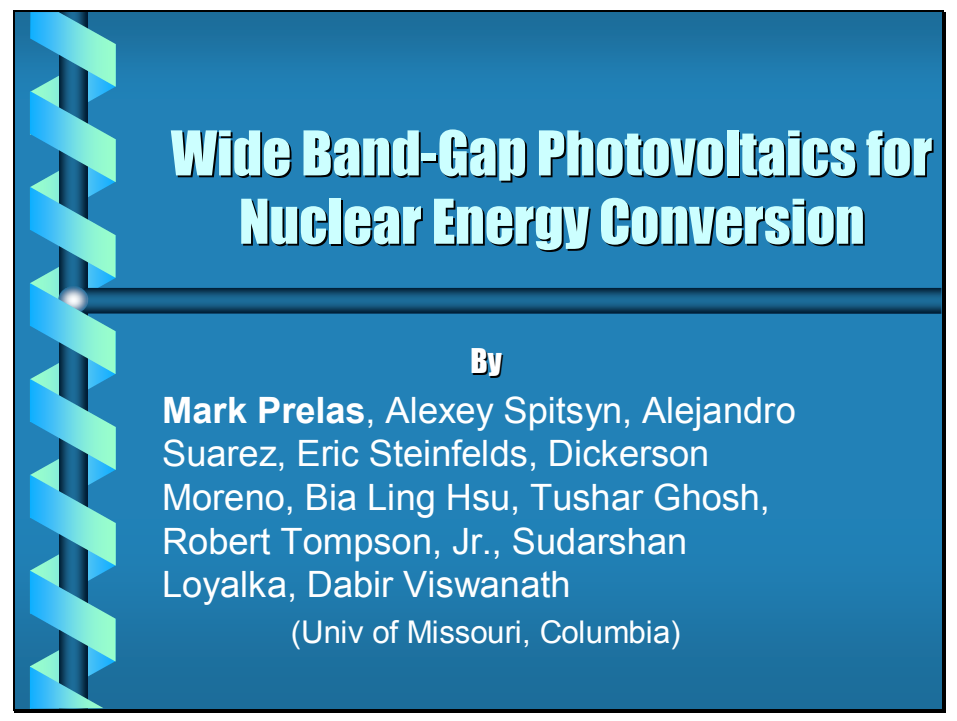


Slide 2

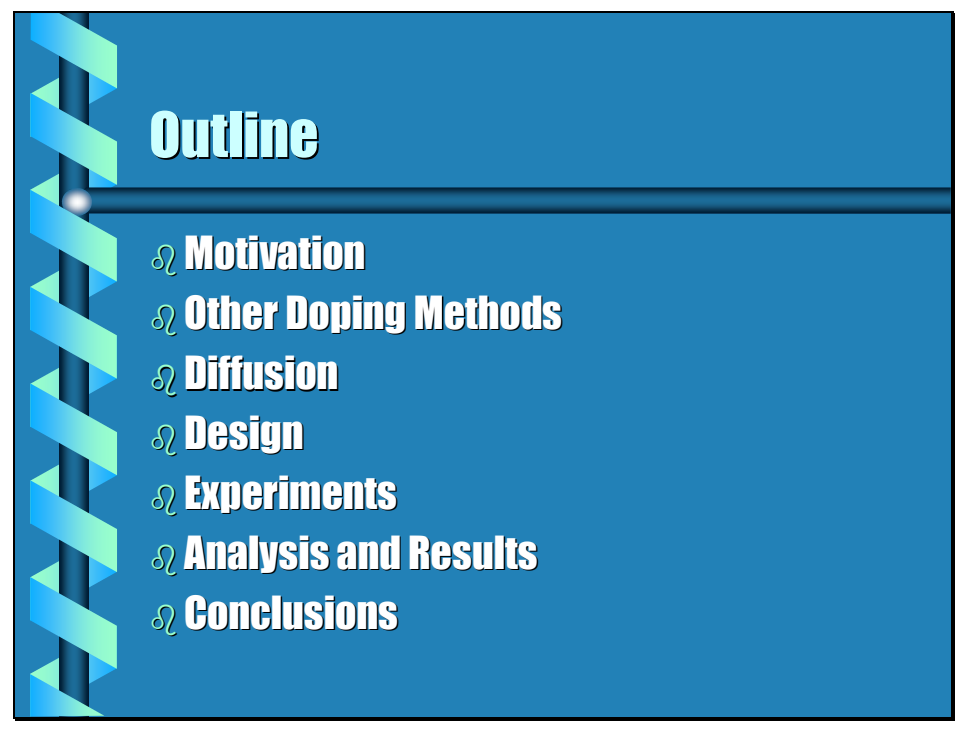


Slide 3

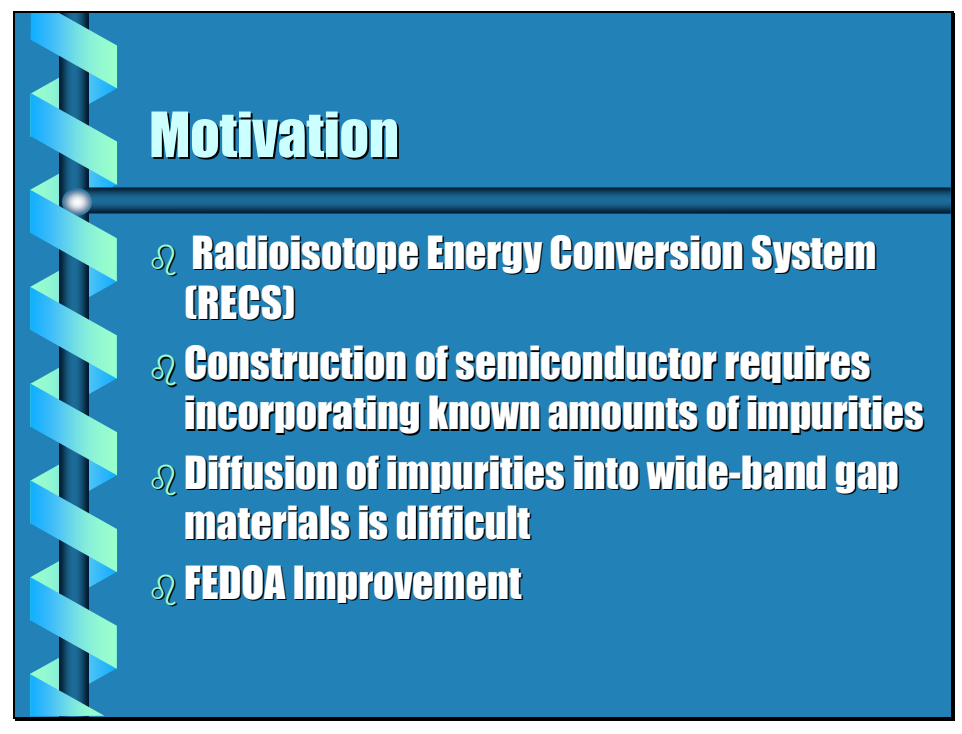


Slide 4

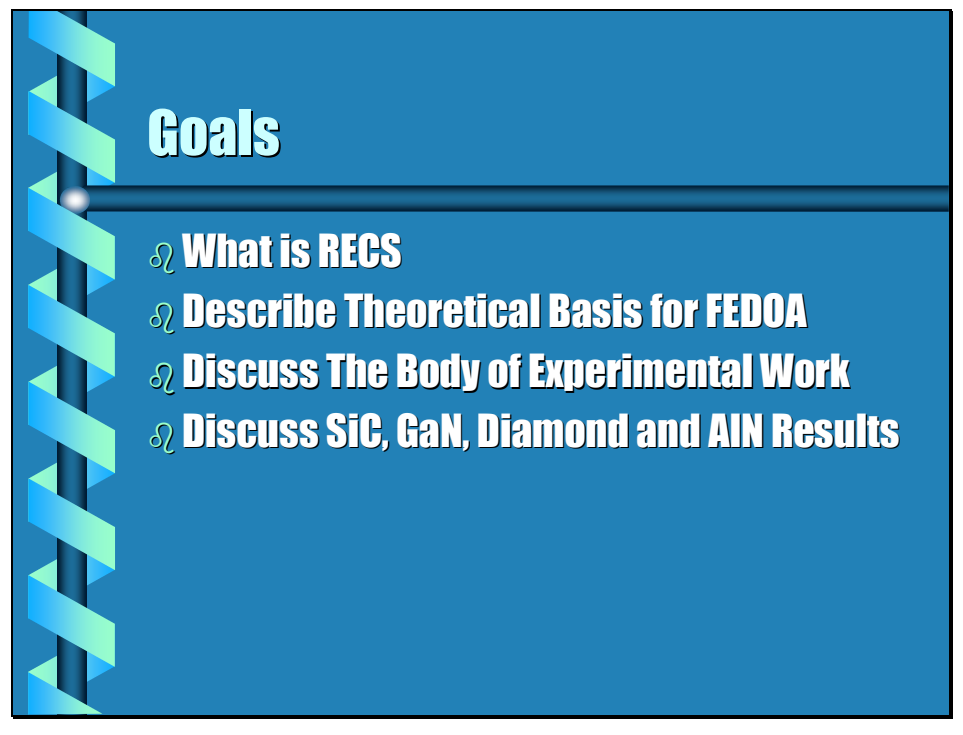


Slide 5

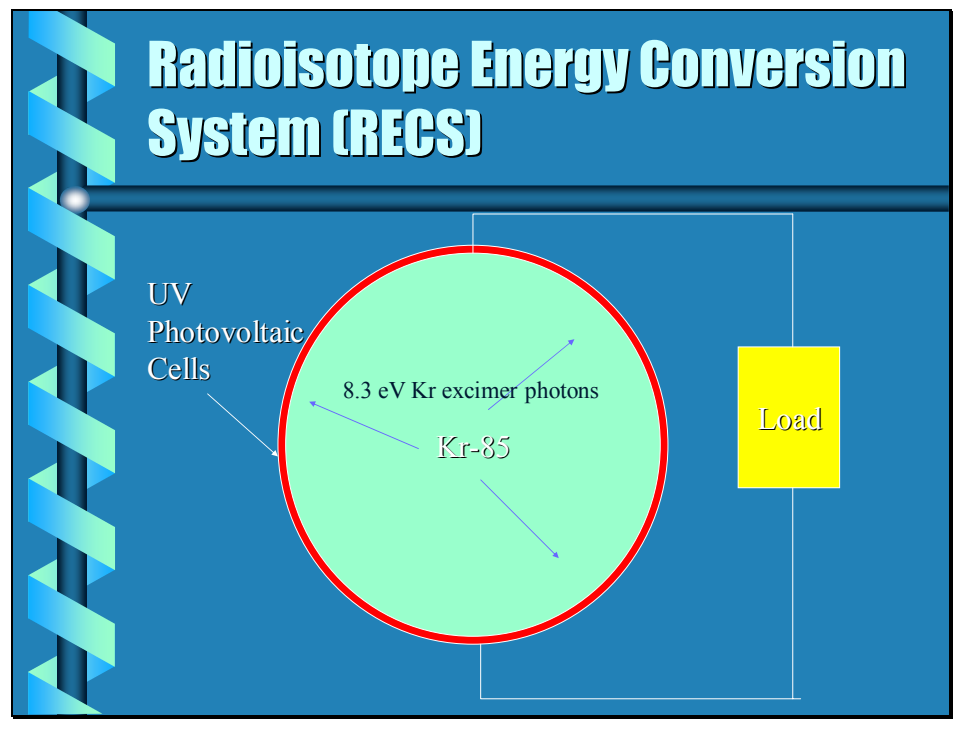


Slide 6

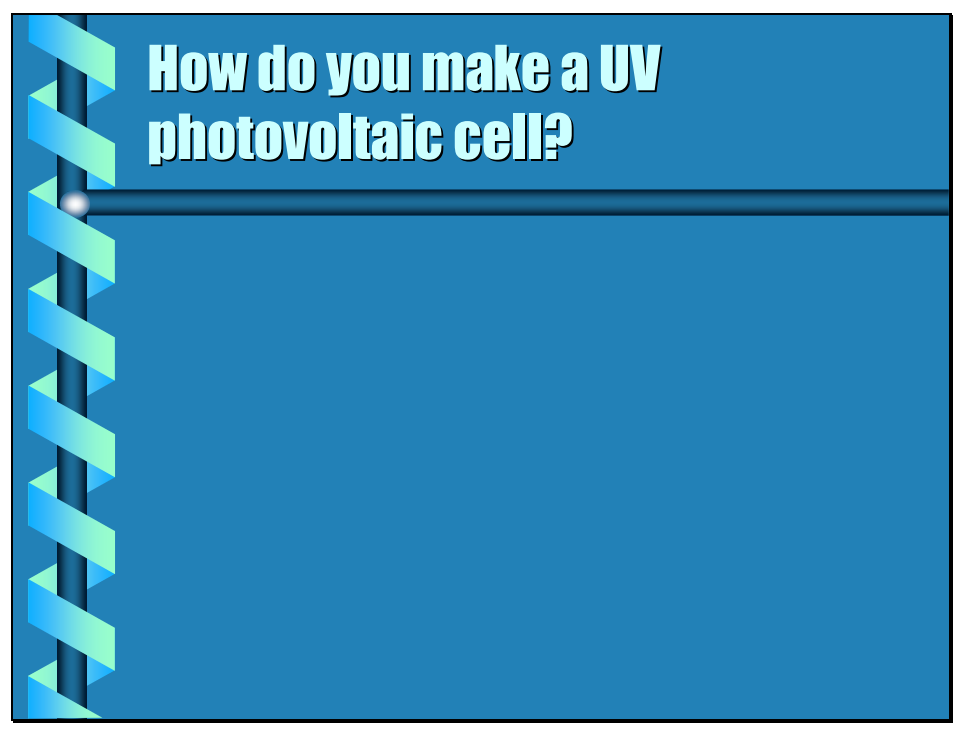


Slide 7

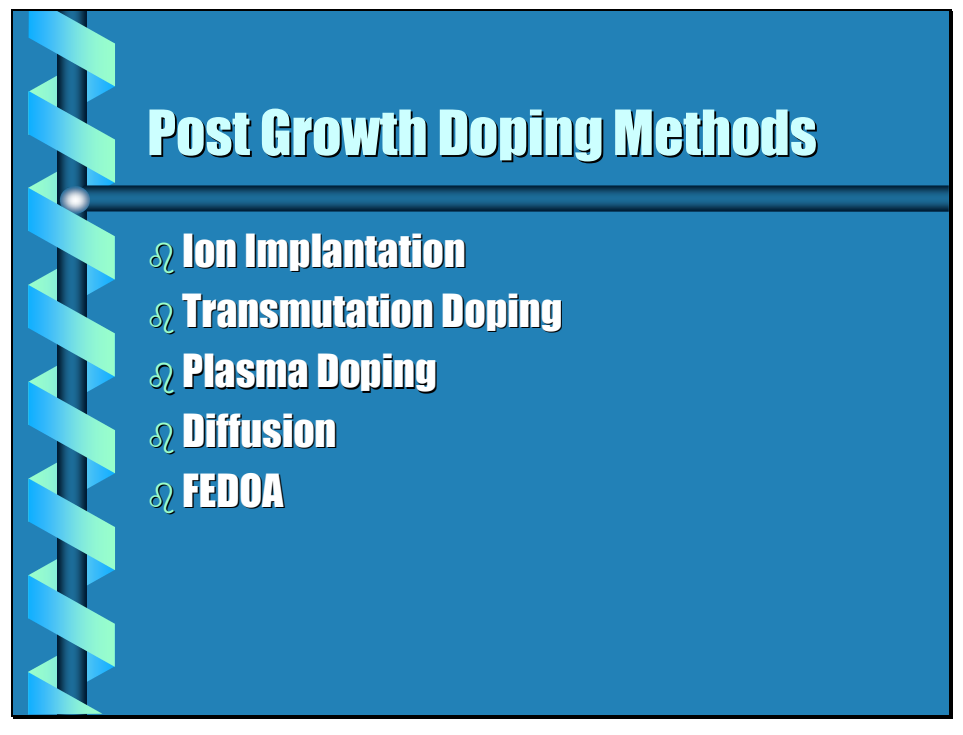


Slide 8

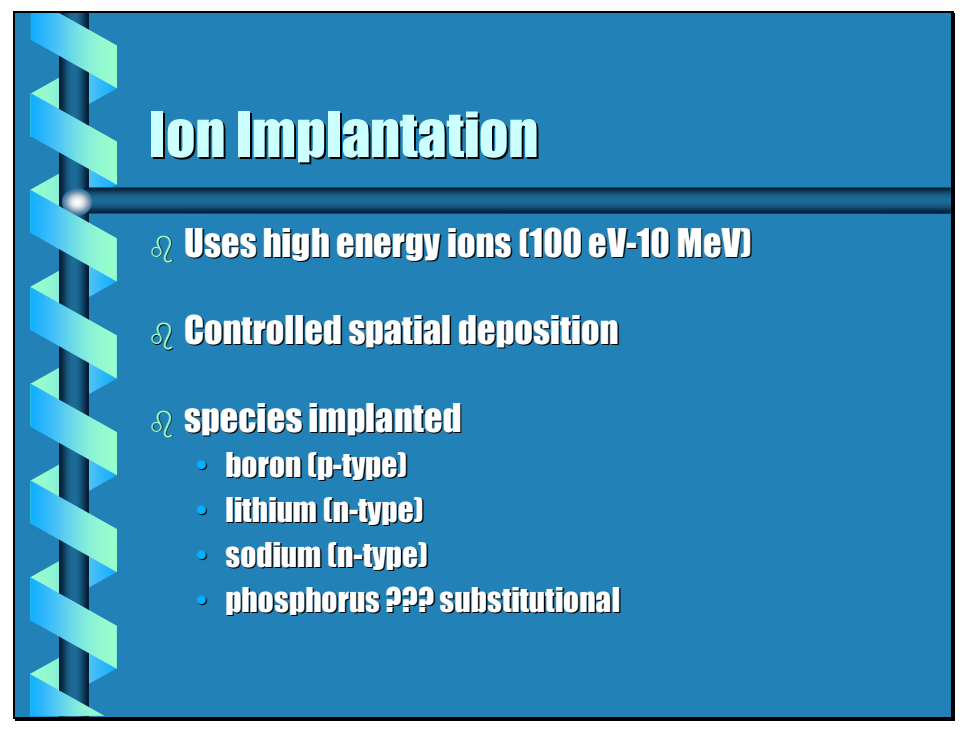


Slide 9

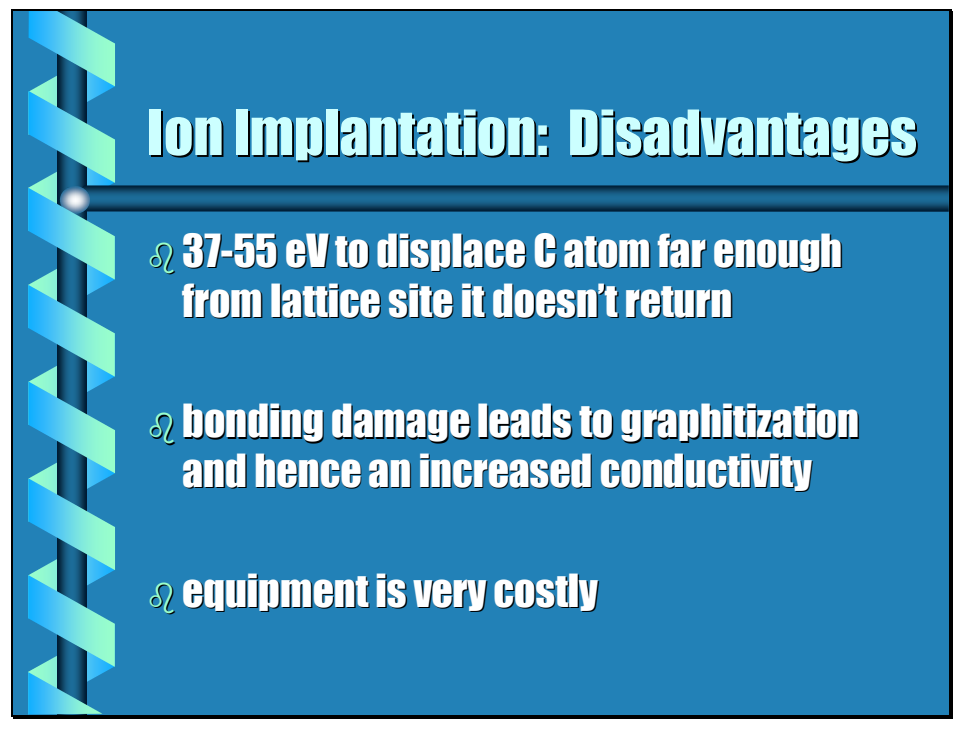


Slide 10

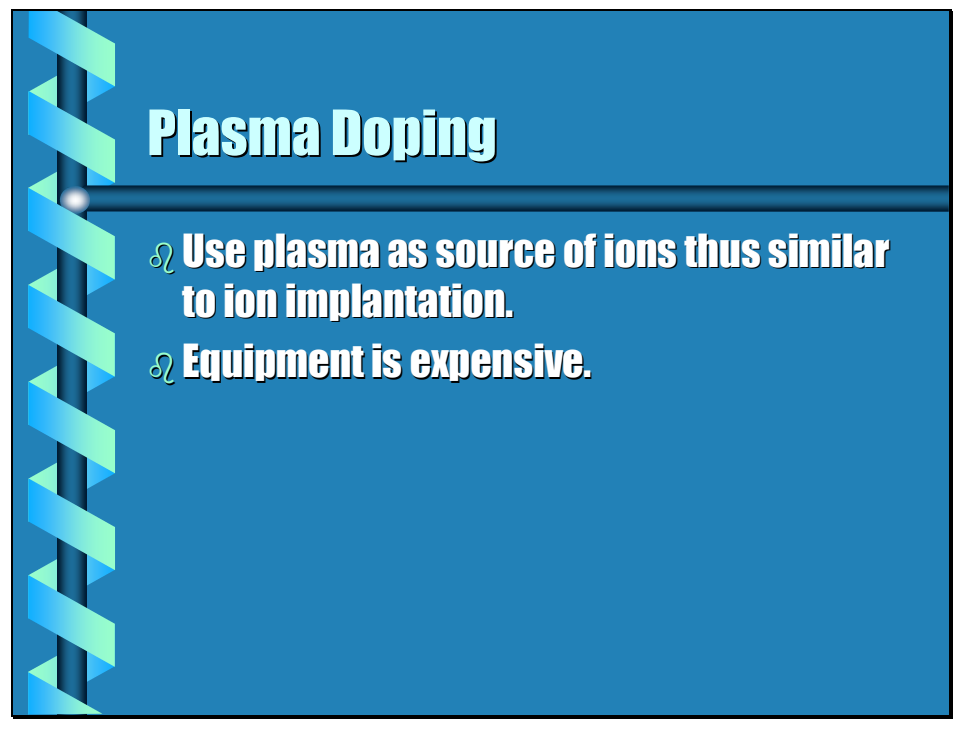


Slide 11

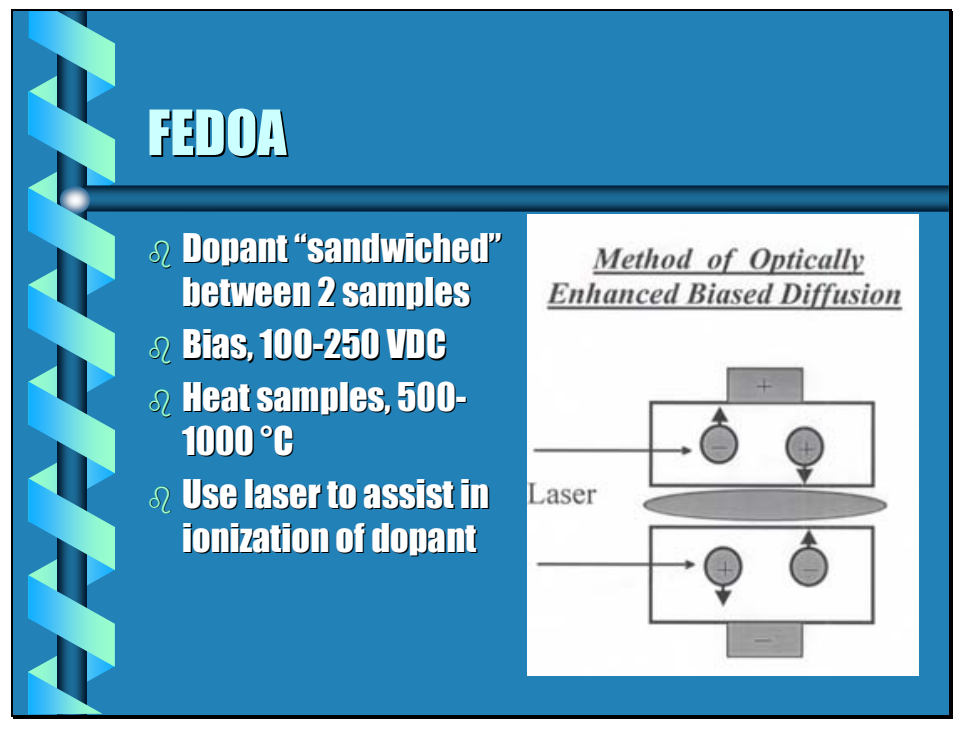


Slide 12

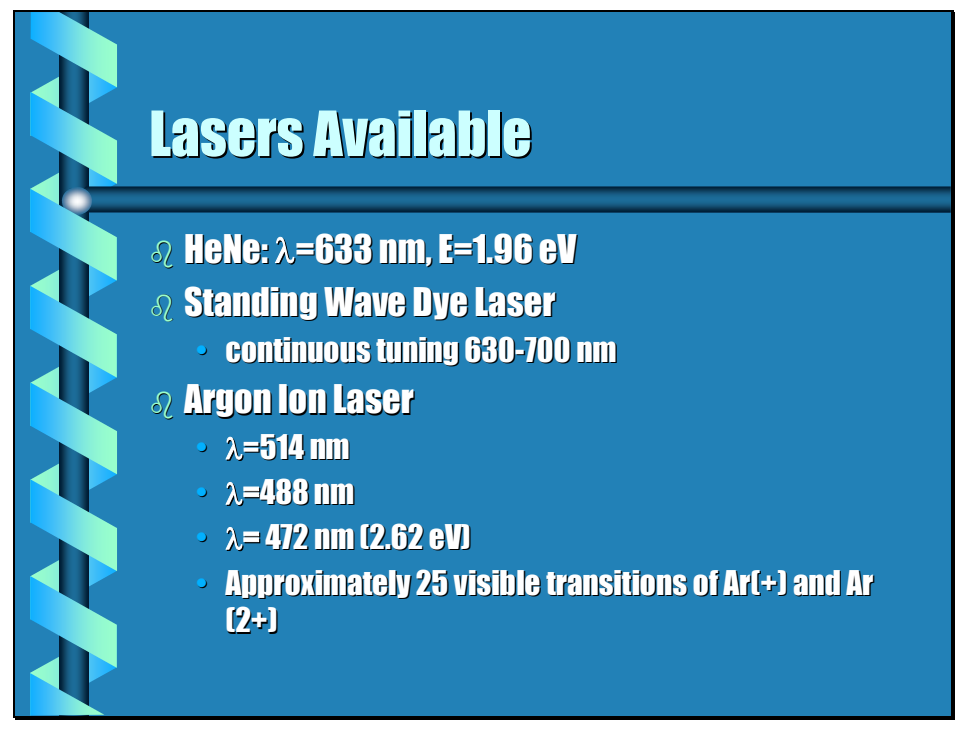


Slide 13

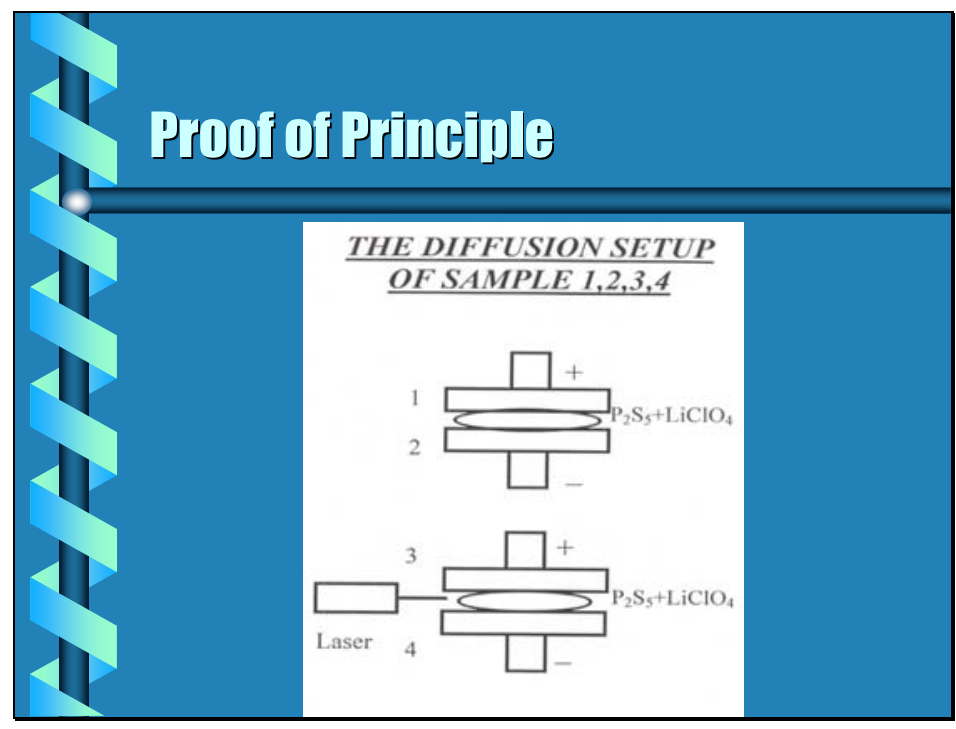


Slide 14

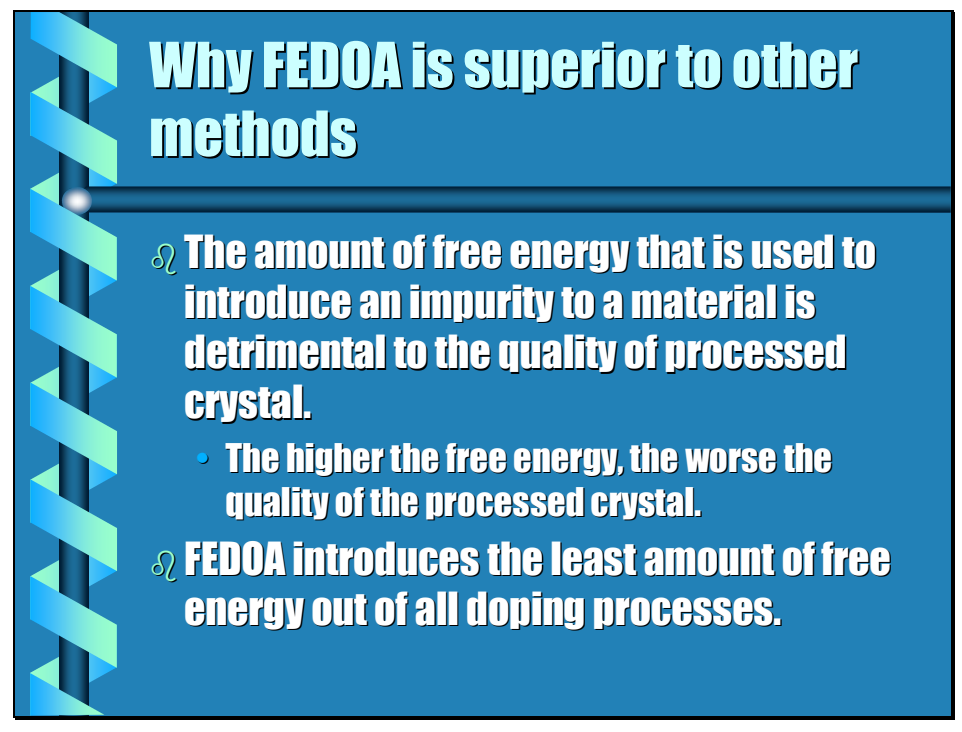


Slide 15

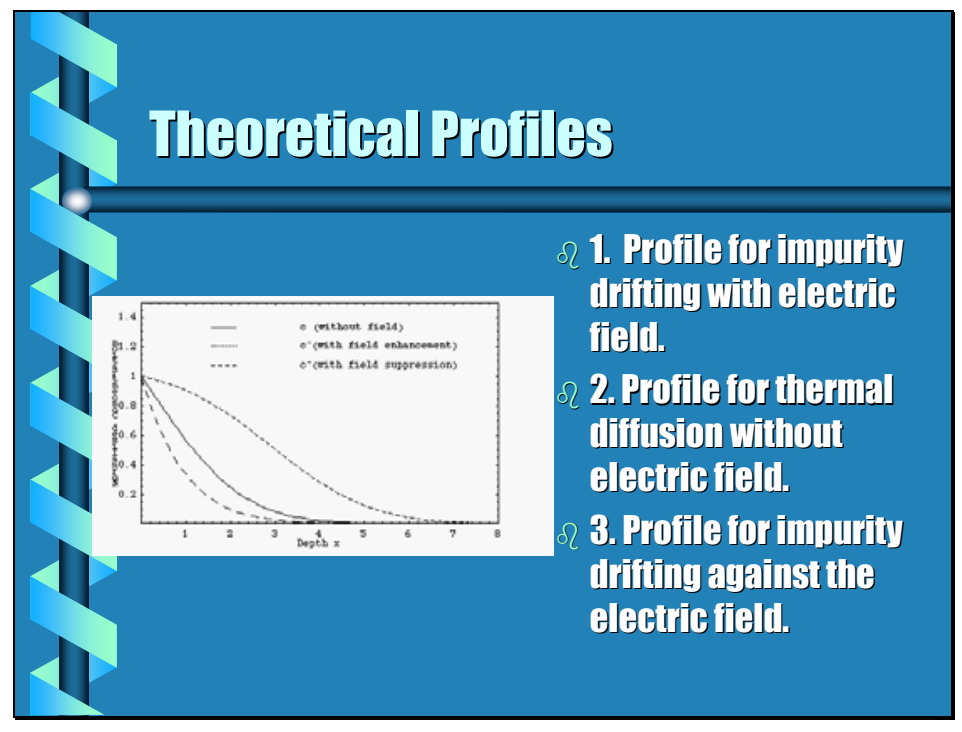


Slide 16

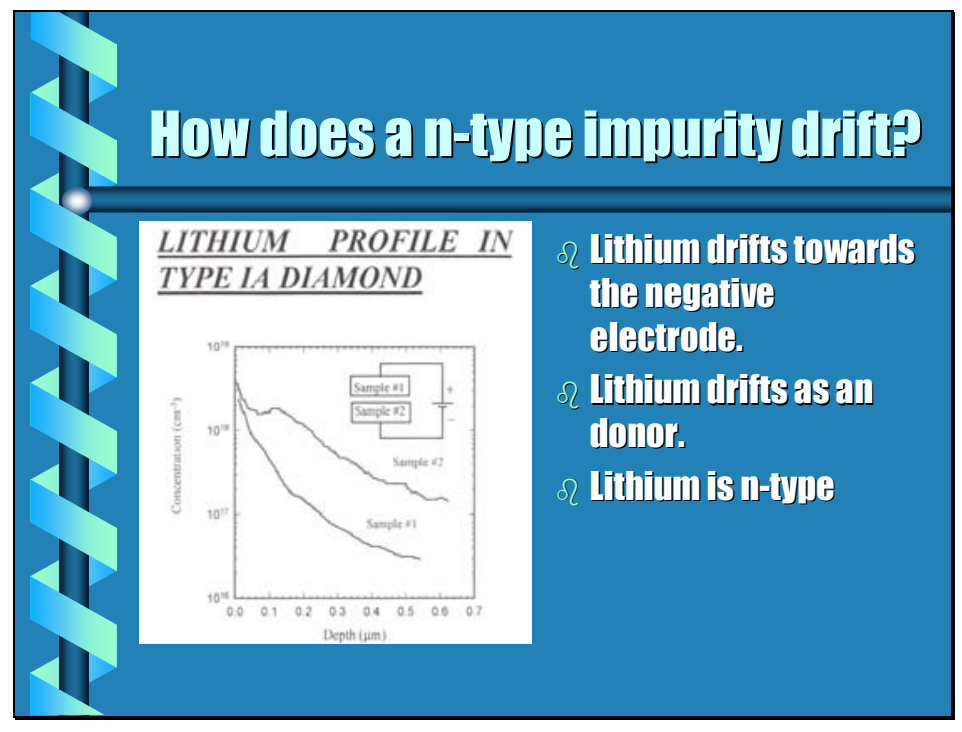


Slide 17

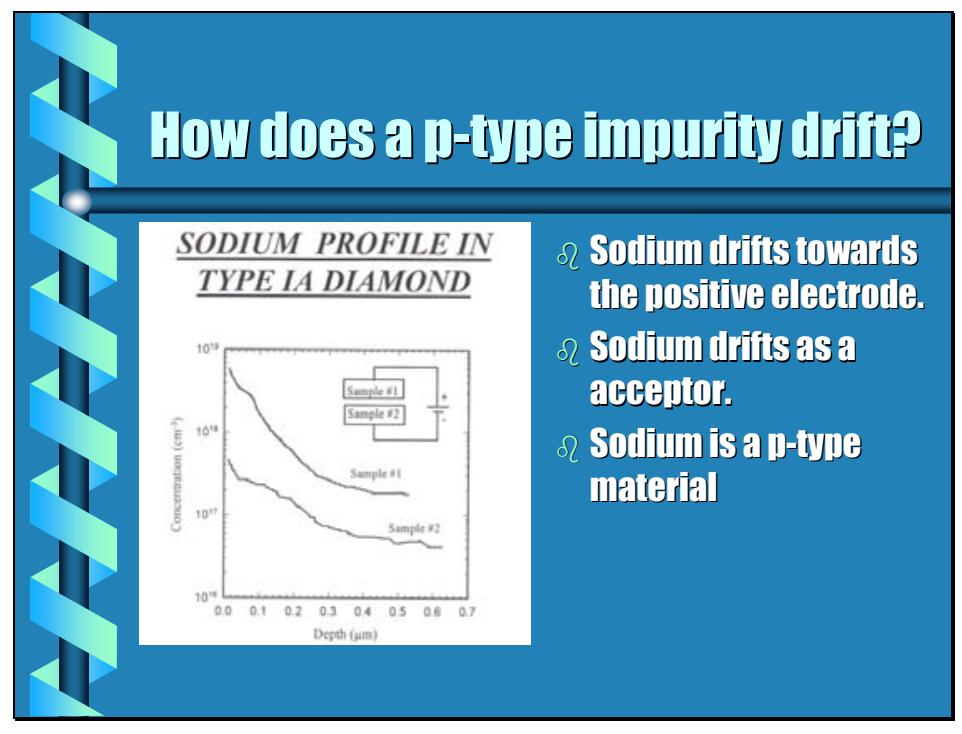


Slide 18

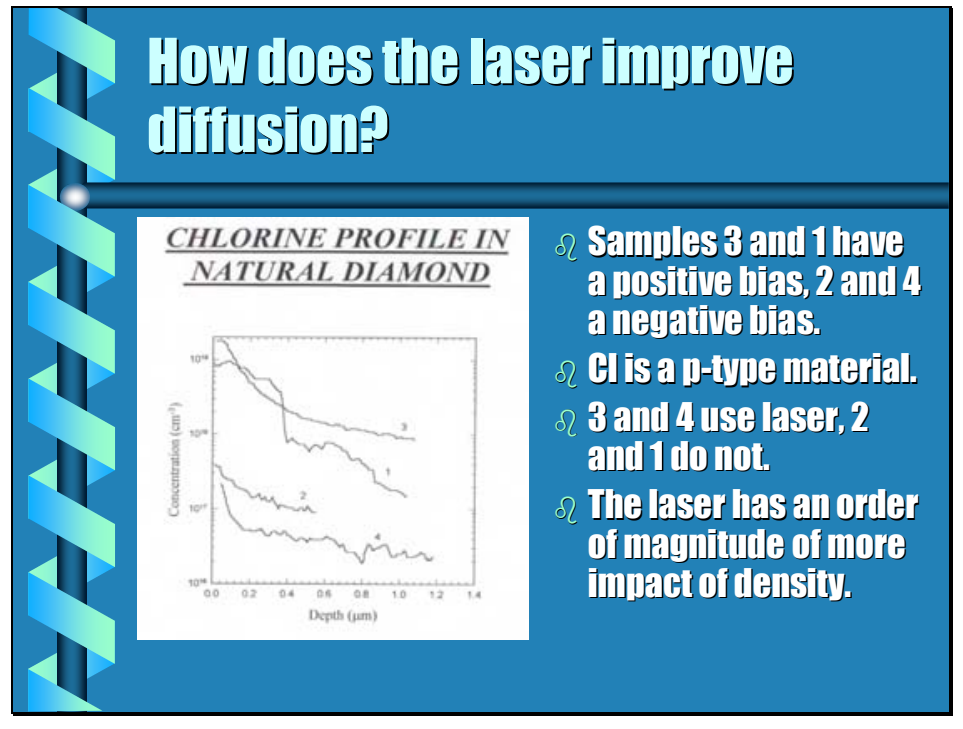


Slide 19

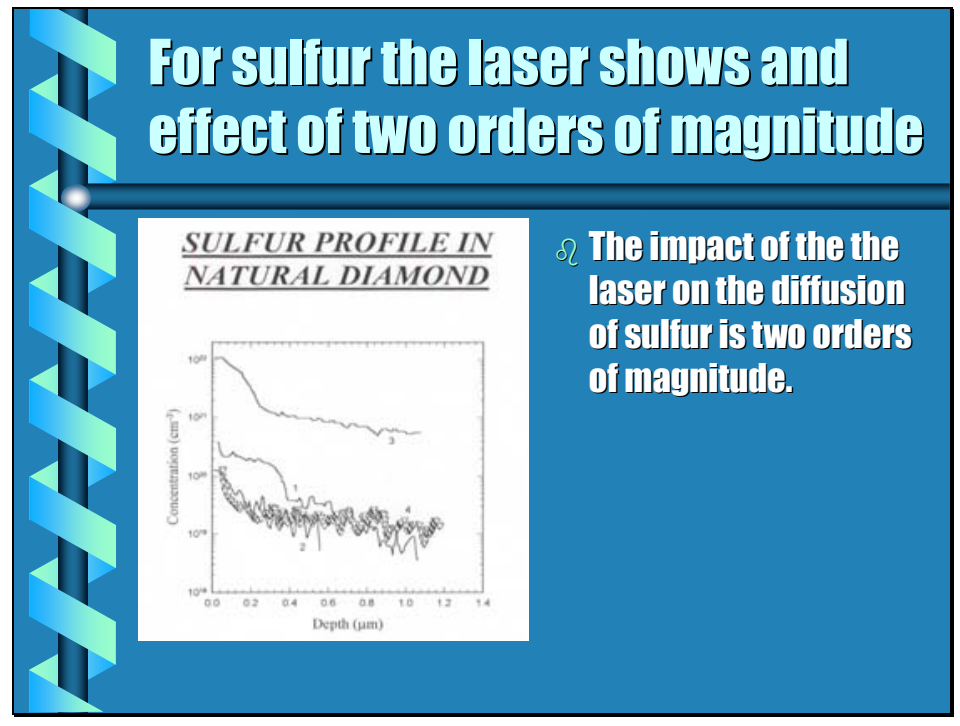


Slide 20

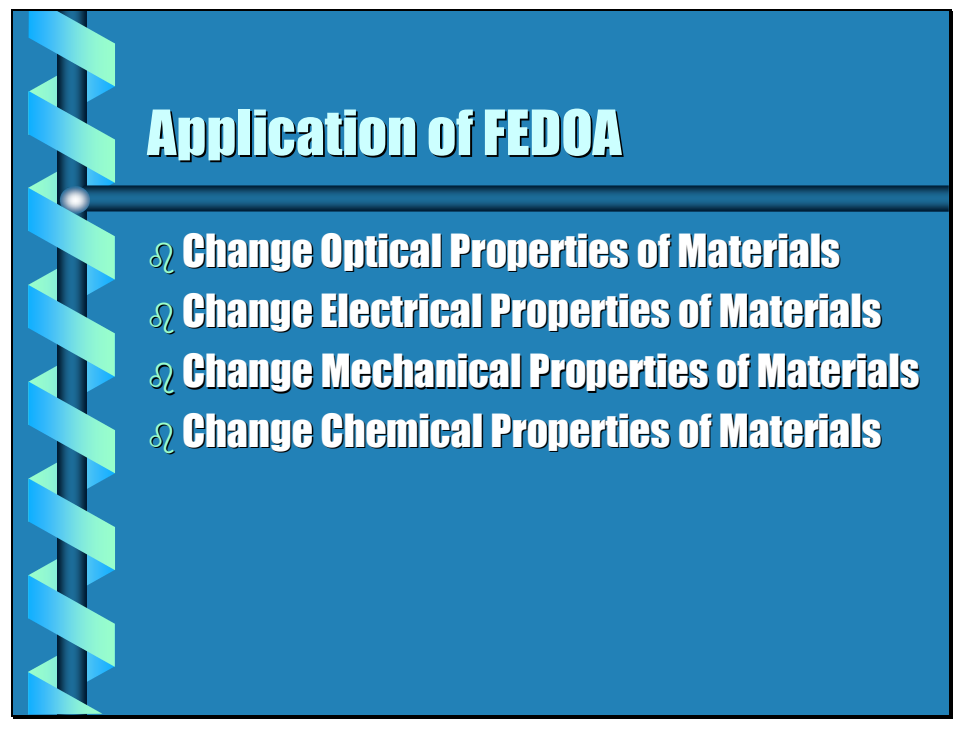


Slide 21

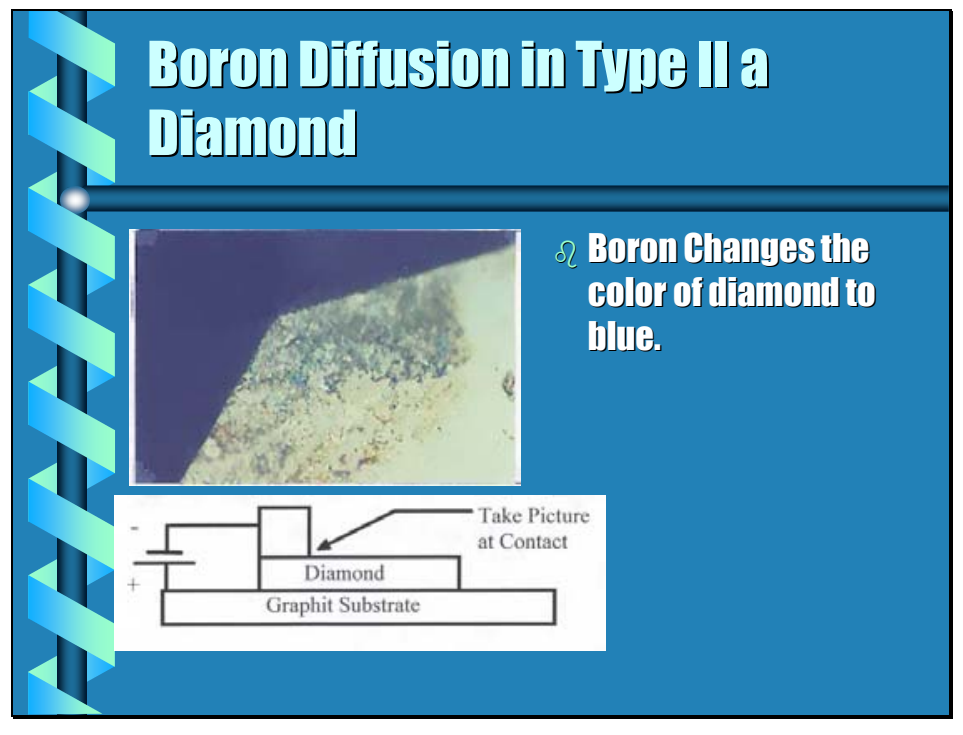


Slide 22

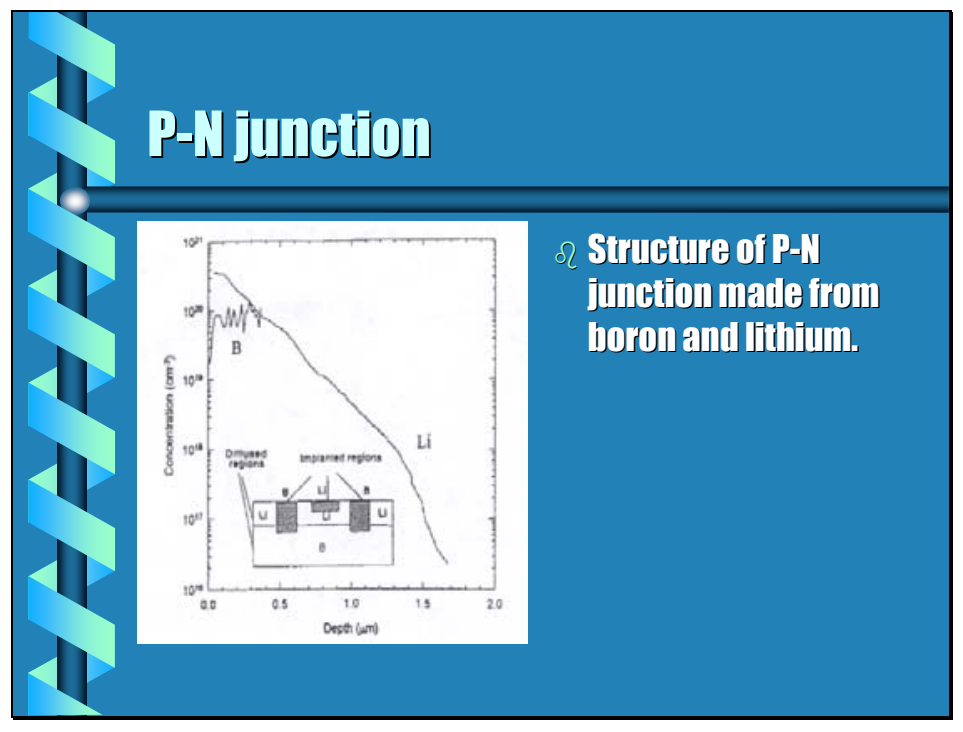


Slide 23

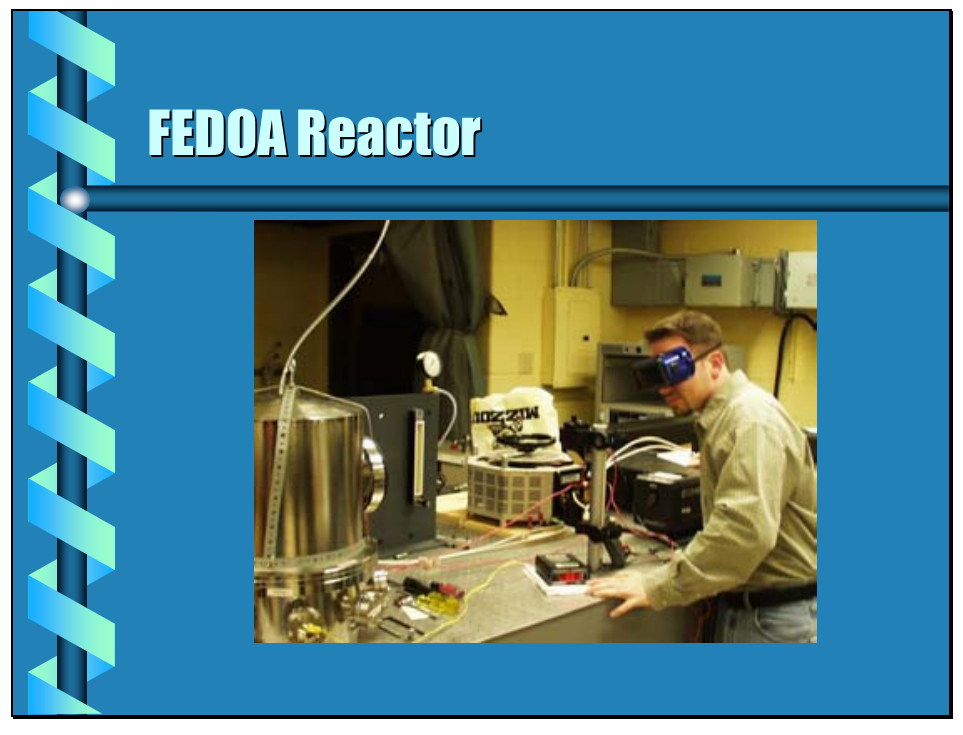


Slide 24

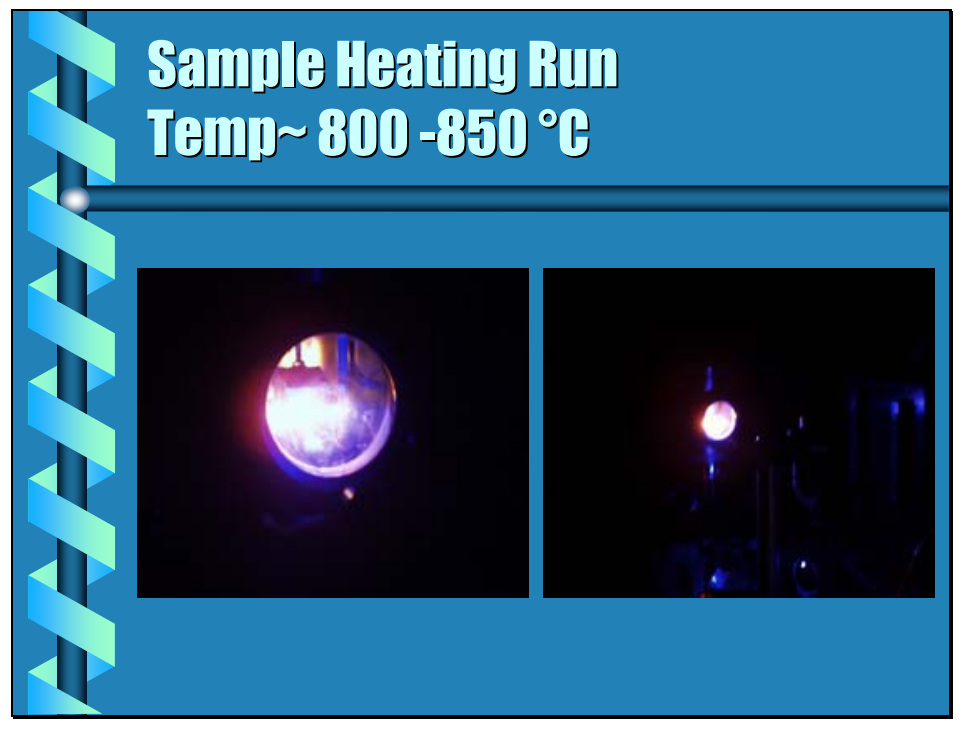


Slide 25

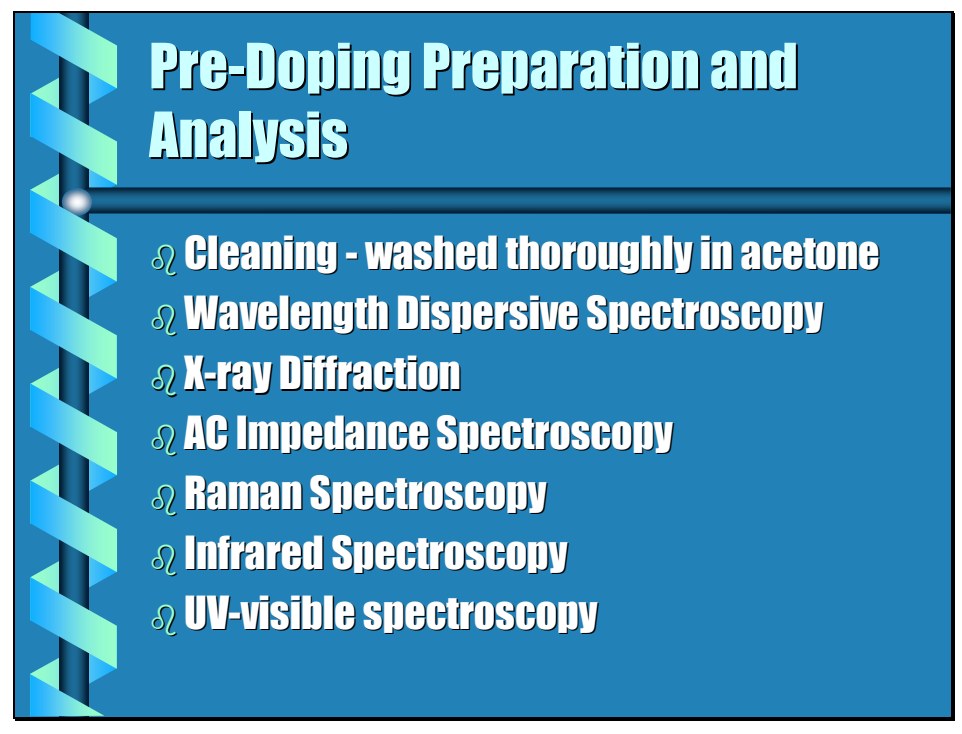


Slide 26

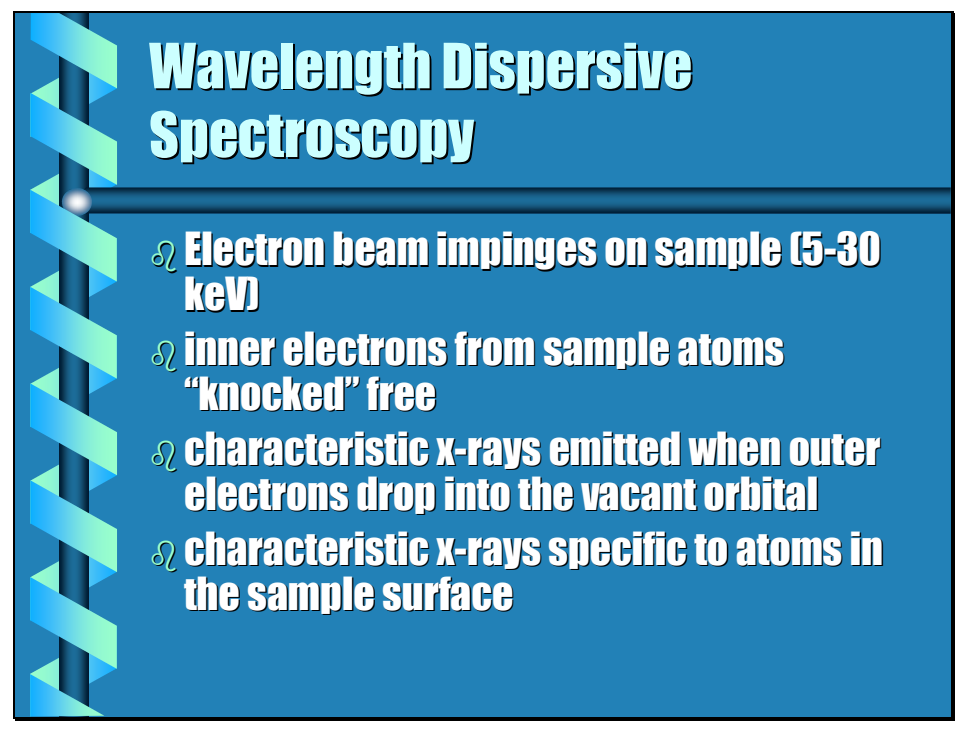


Slide 27

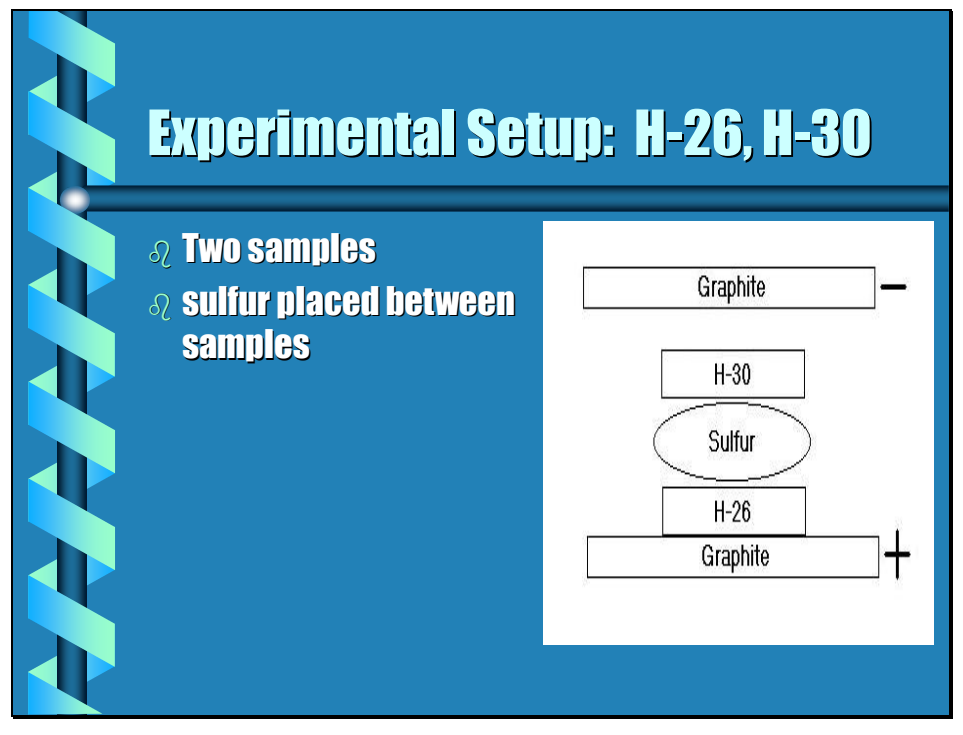


Slide 28

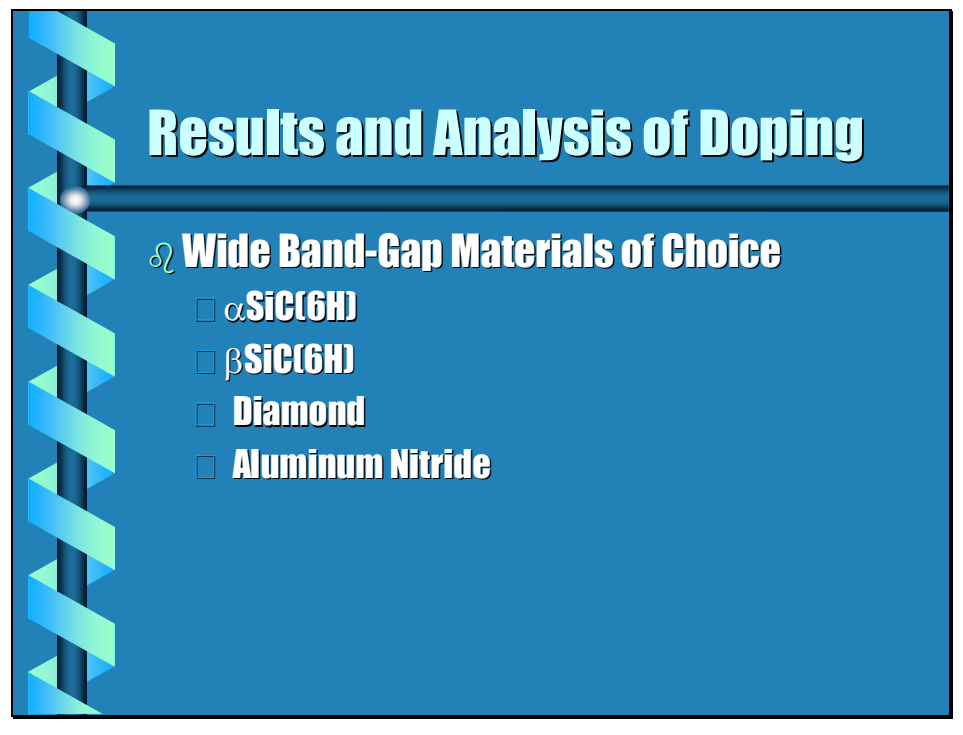


Slide 29

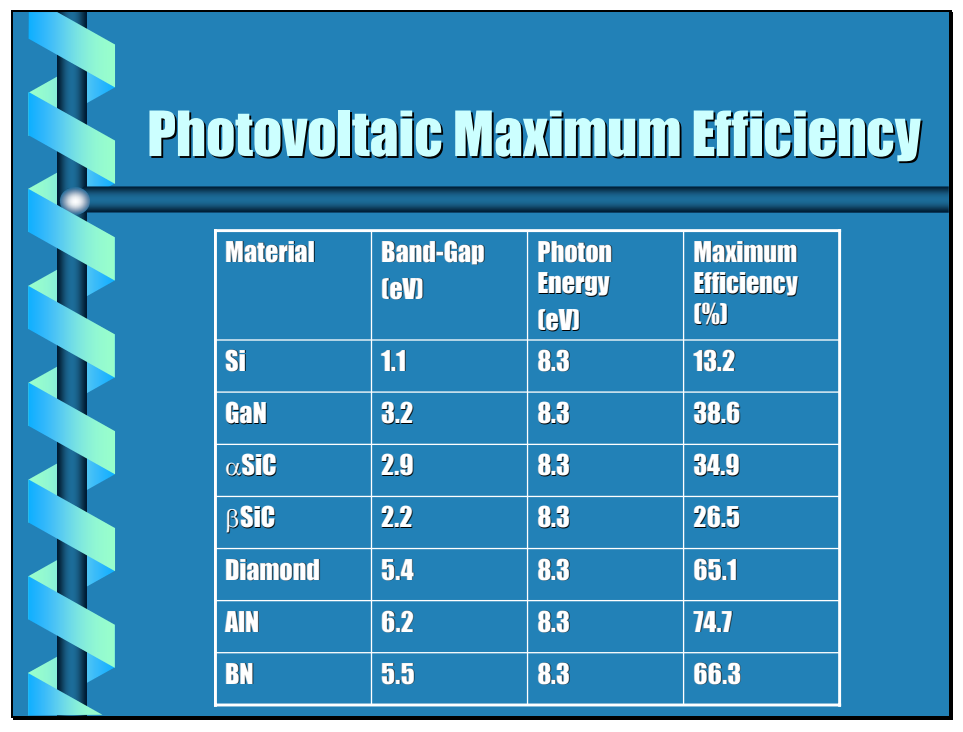


Slide 30

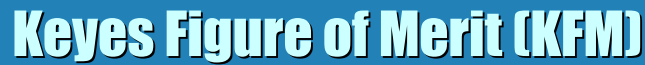

or The leyes figdre of merit tales into aceount the DOWOr donsity dissipation for olosely paster integratoil oirentis. Ifigh thermal eondurtivity is an important olement for the Lloyes figure of merith layes figdre of merit is haseri on tsat $\sigma_{t}$

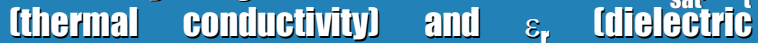

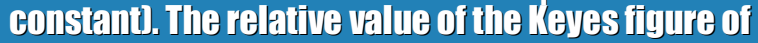
merit is the speri of the transistor in the material.

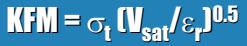


Slide 31

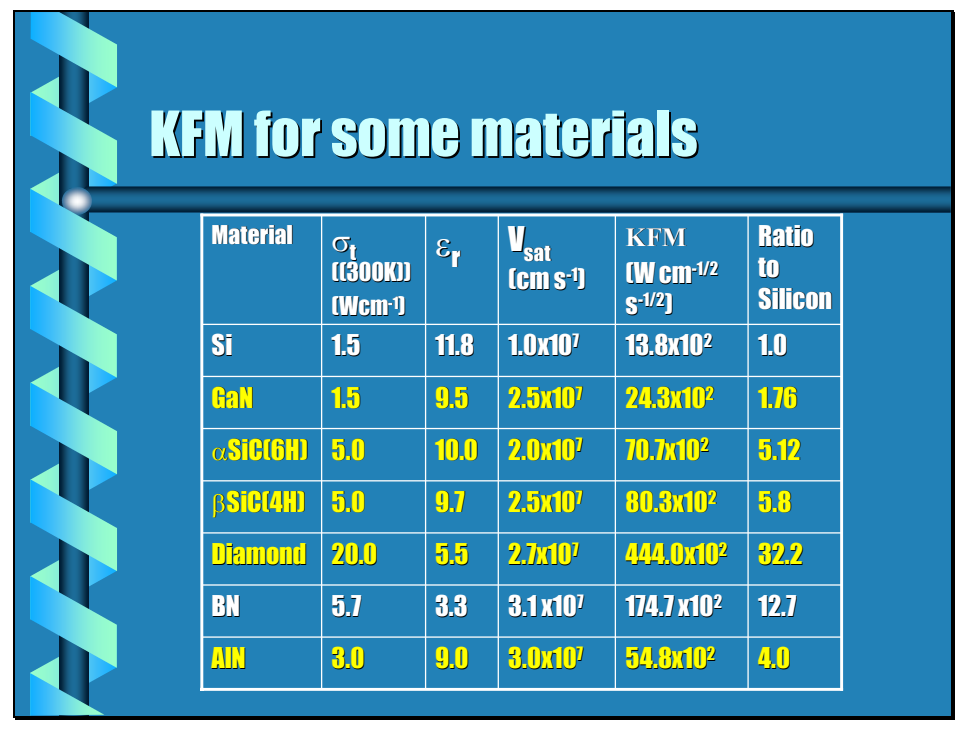


Slide 32

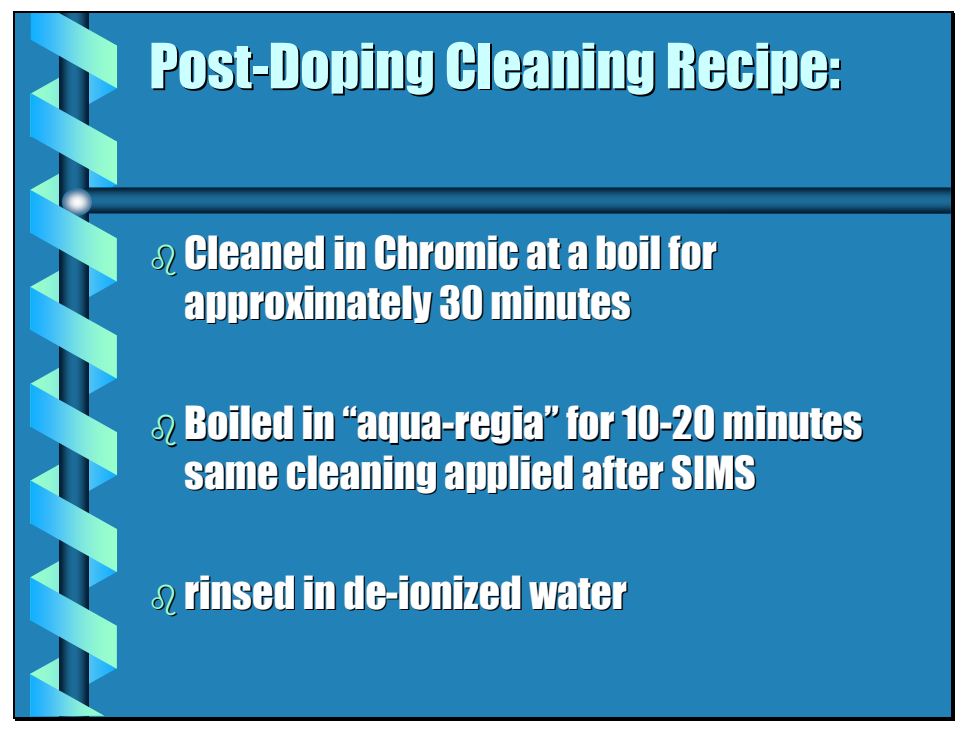


Slide 33

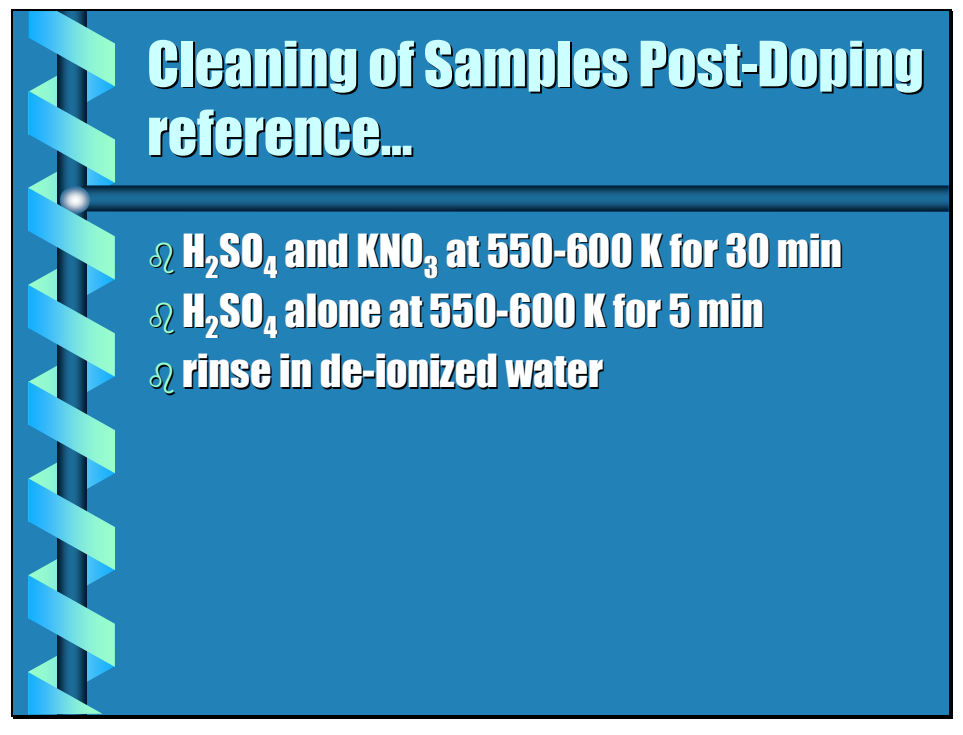


Slide 34

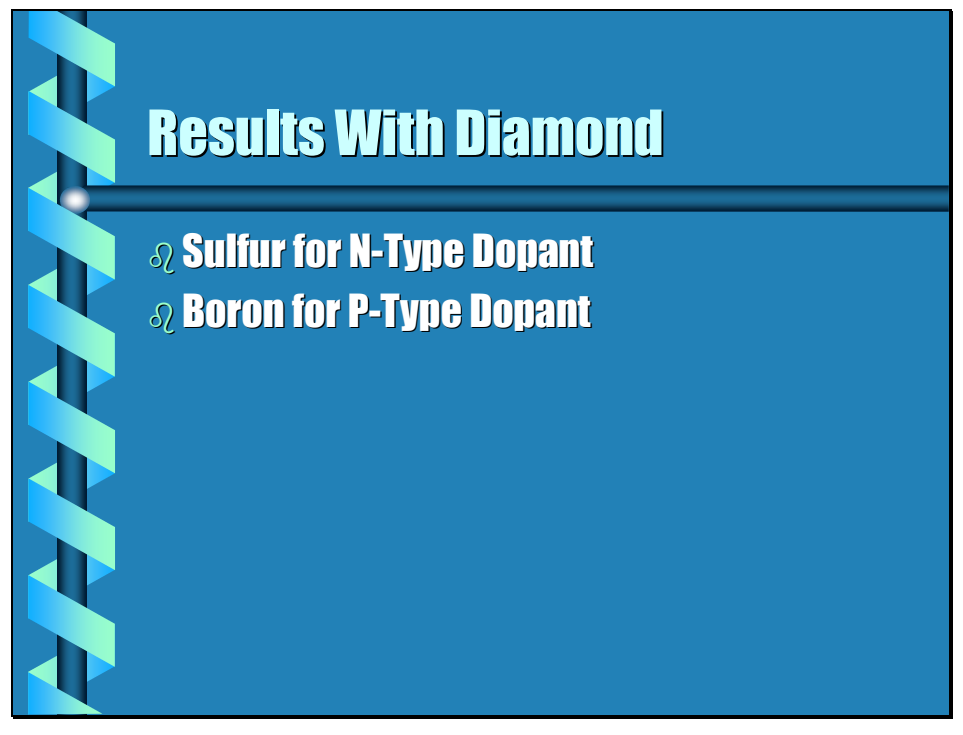


Slide 35

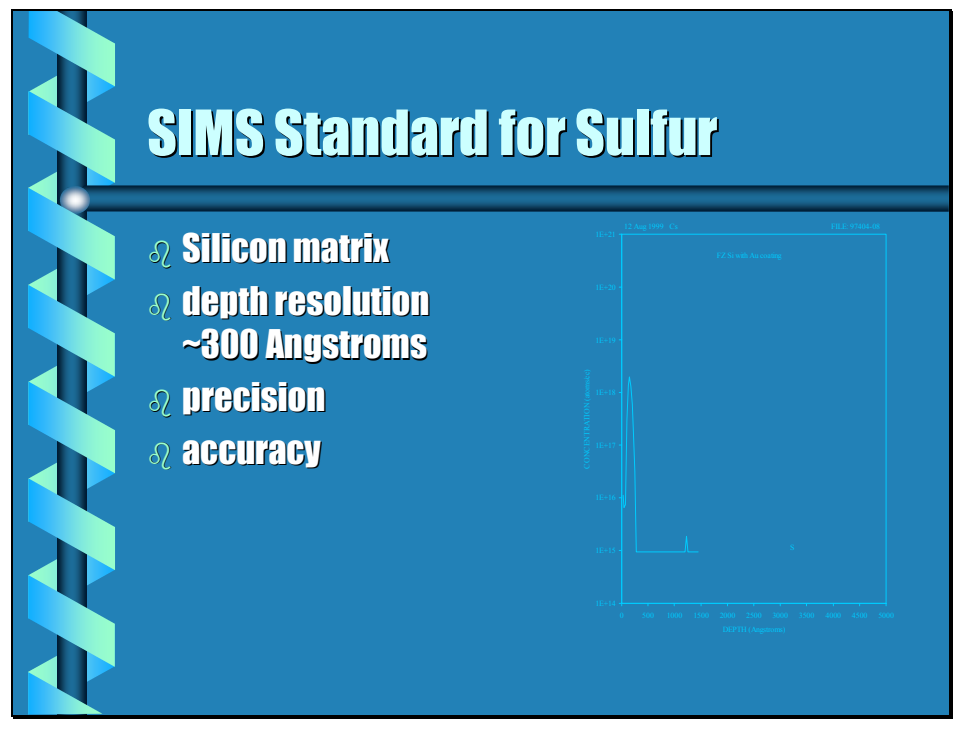


Slide 36

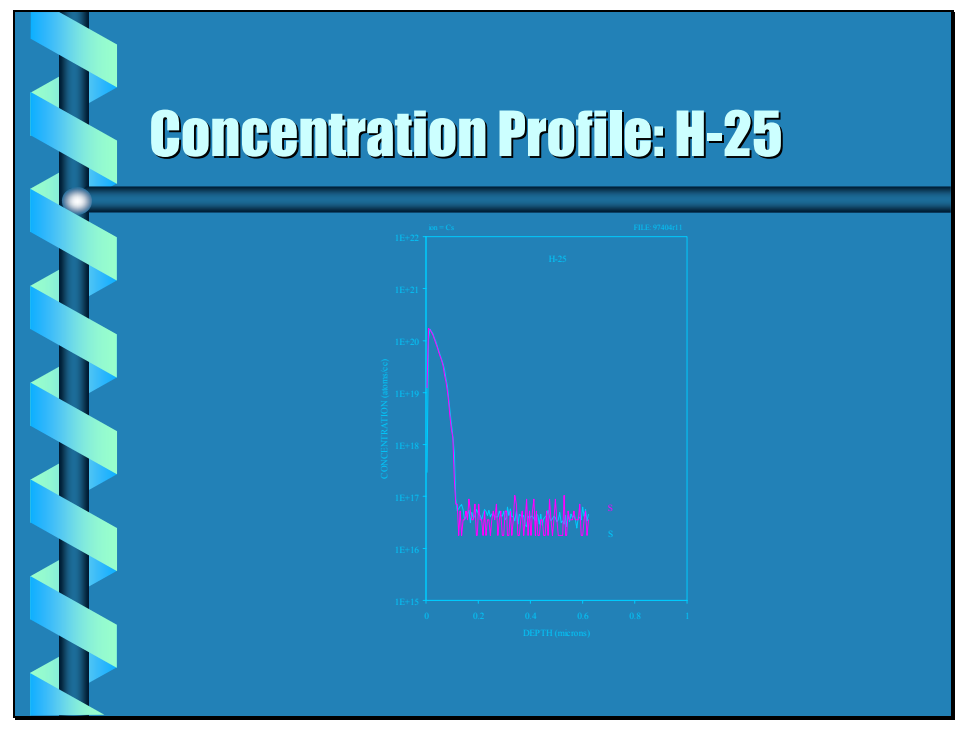


Slide 37

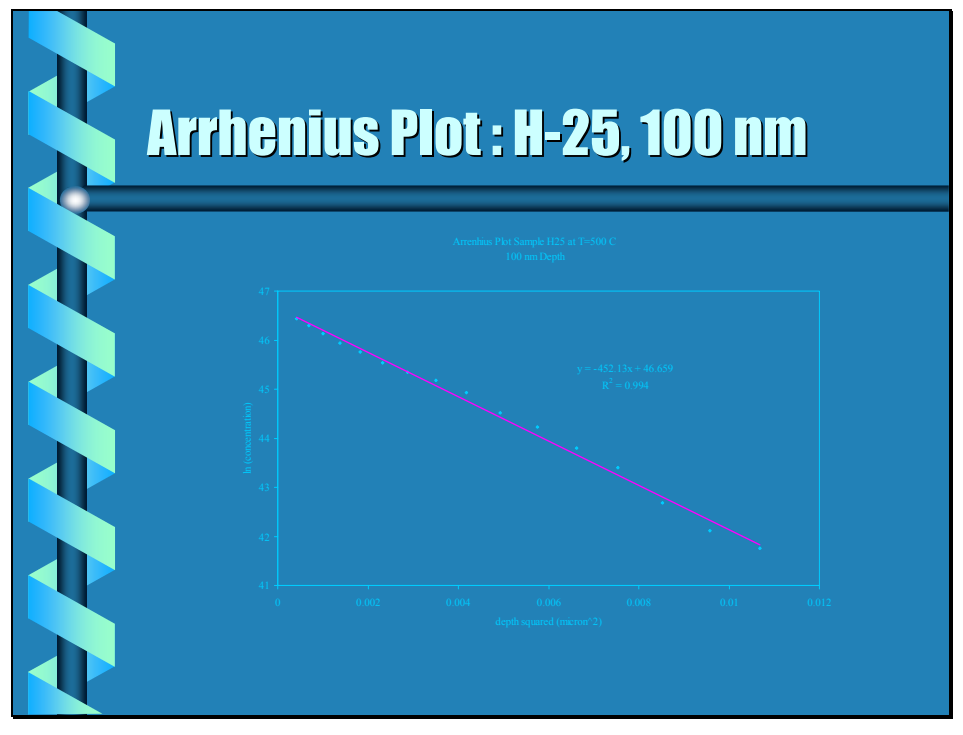


Slide 38

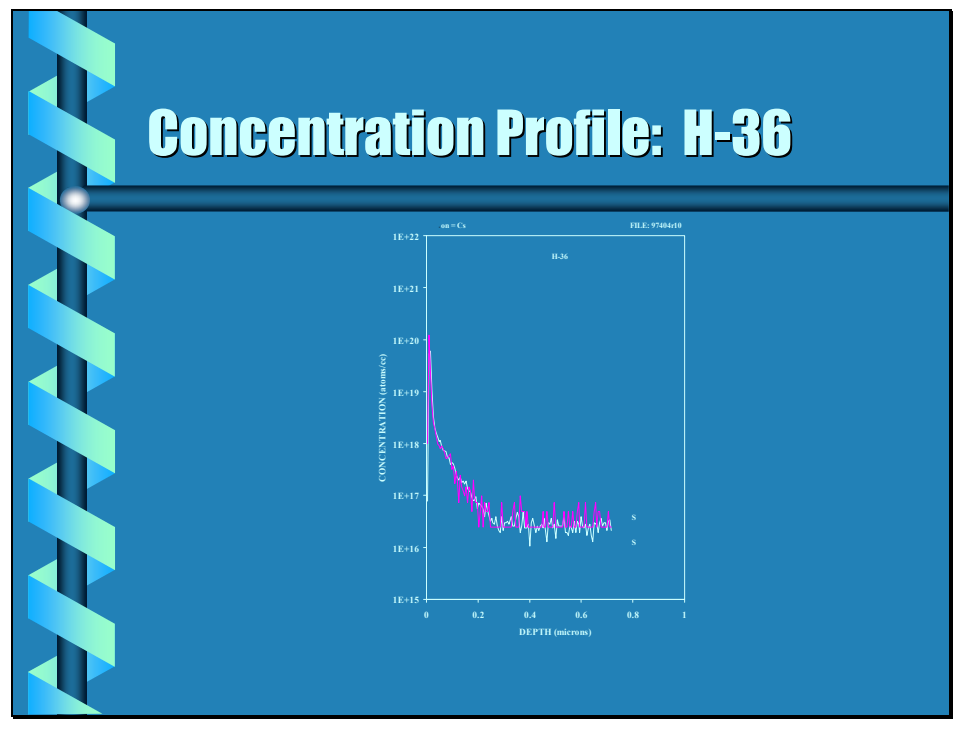


Slide 39

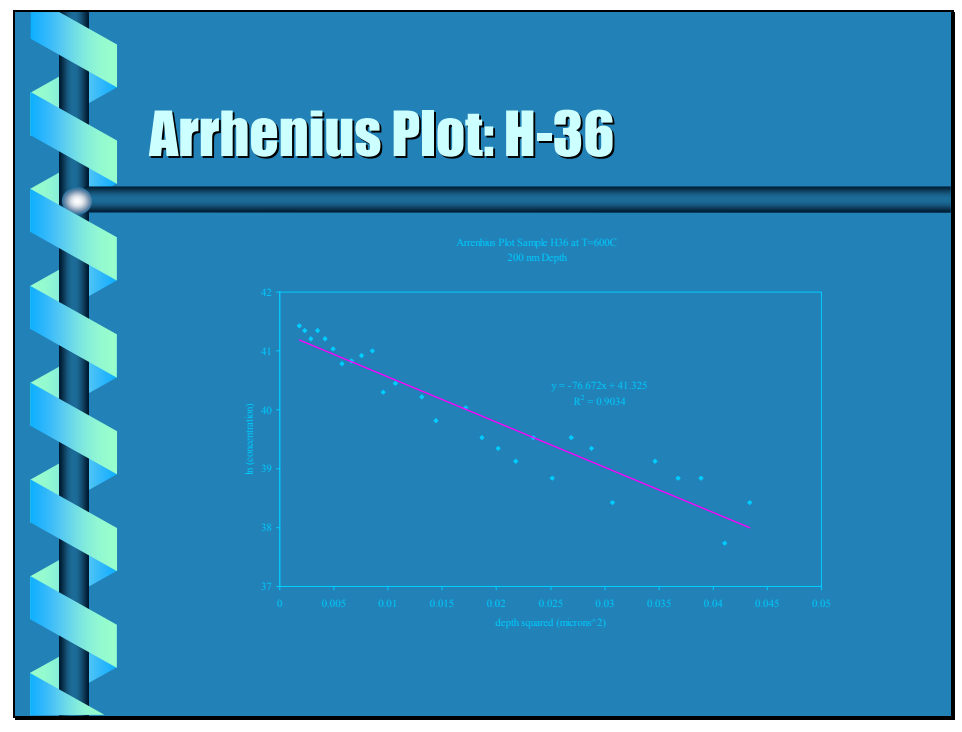


Slide 40

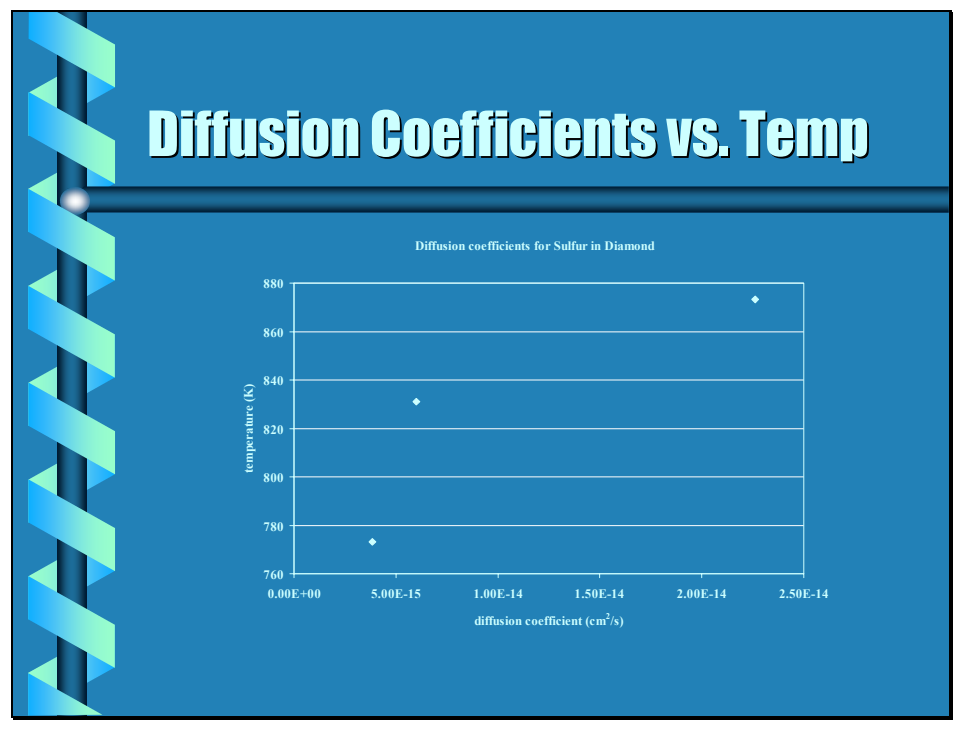


Slide 41

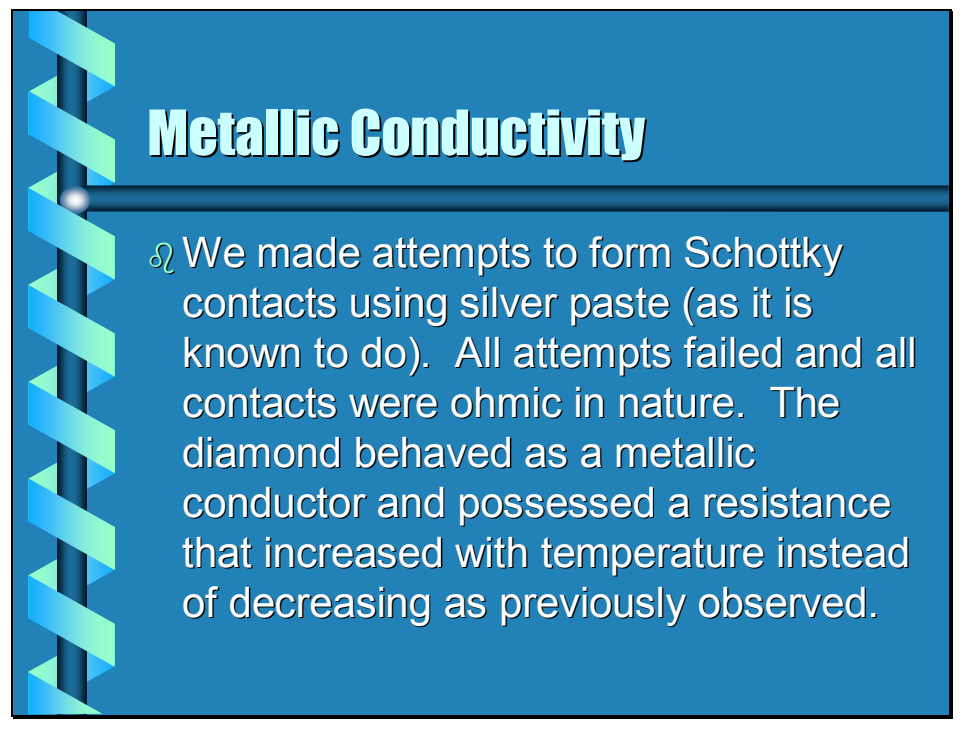


Slide 42

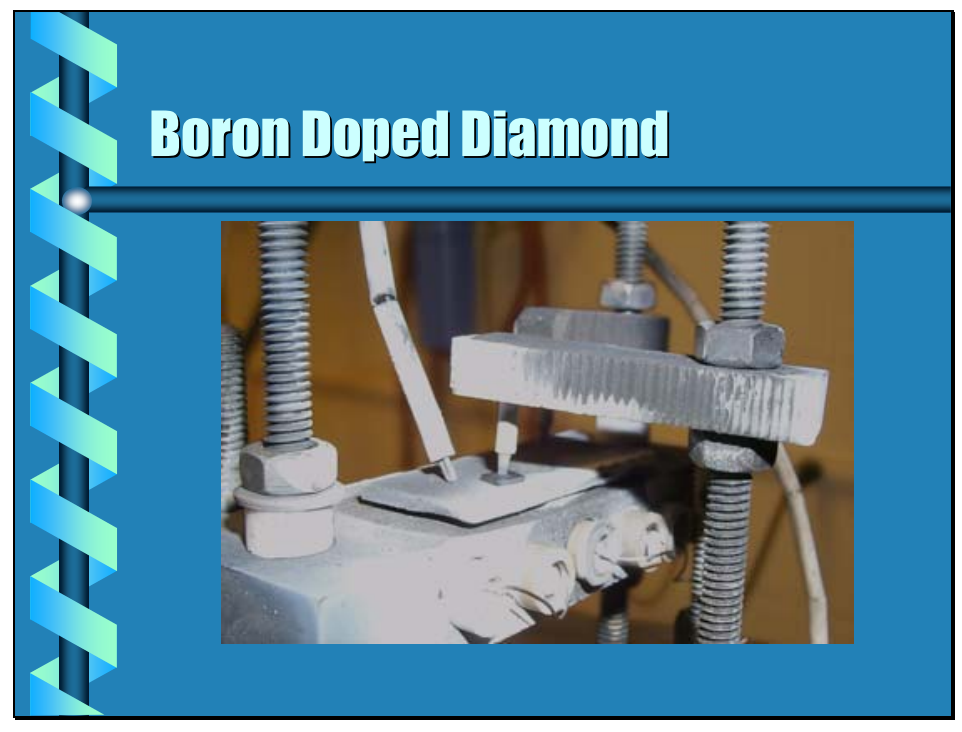


Slide 43

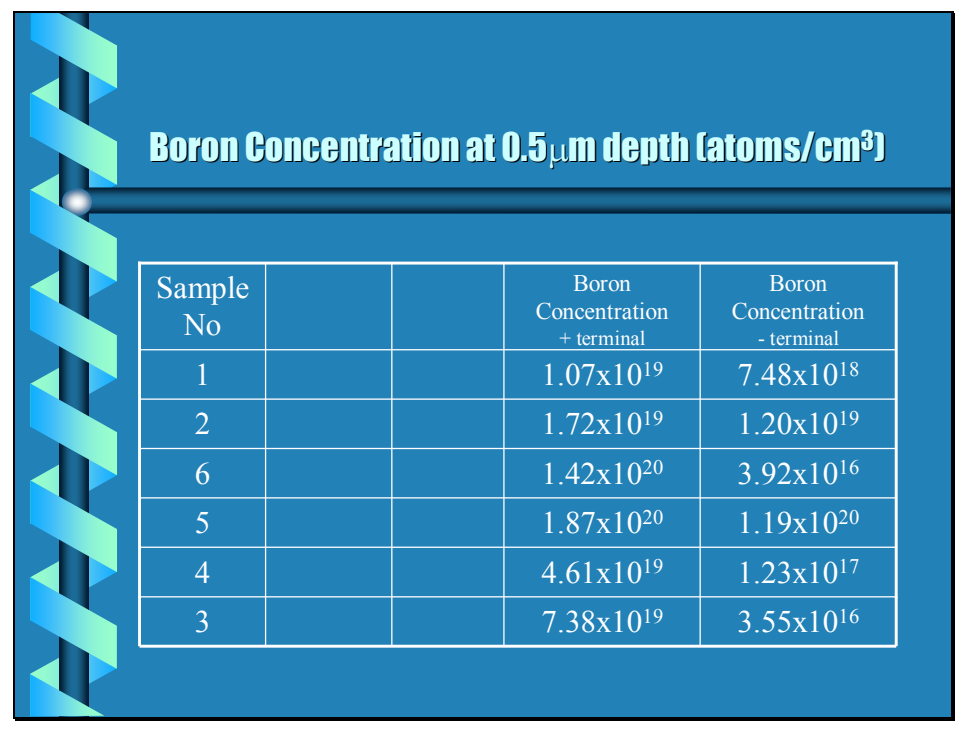


Slide 44

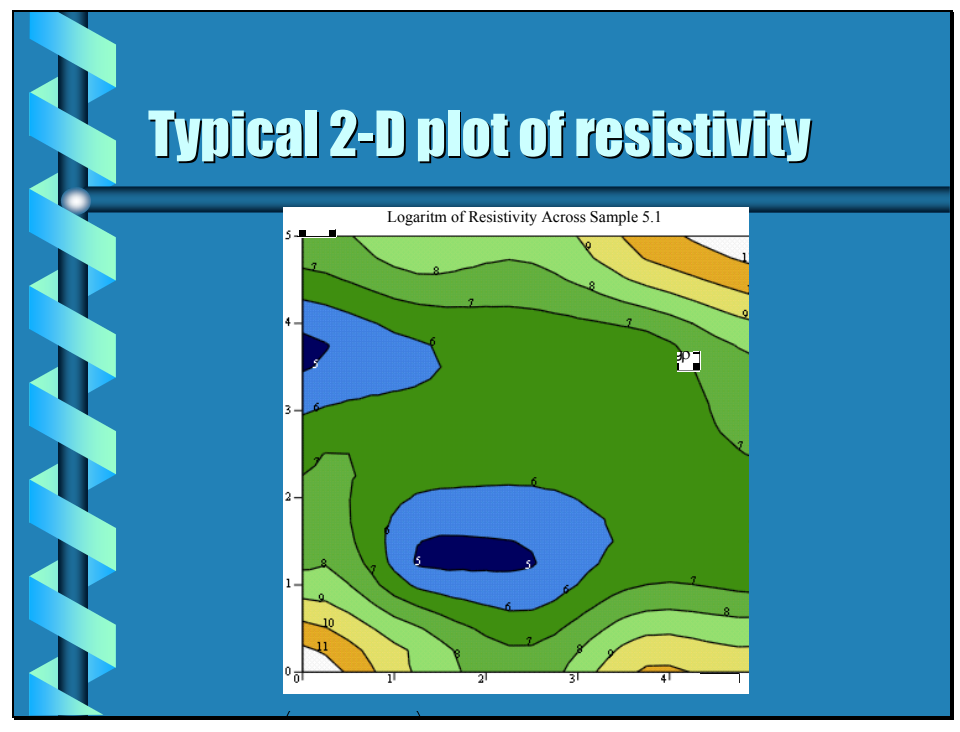


Slide 45

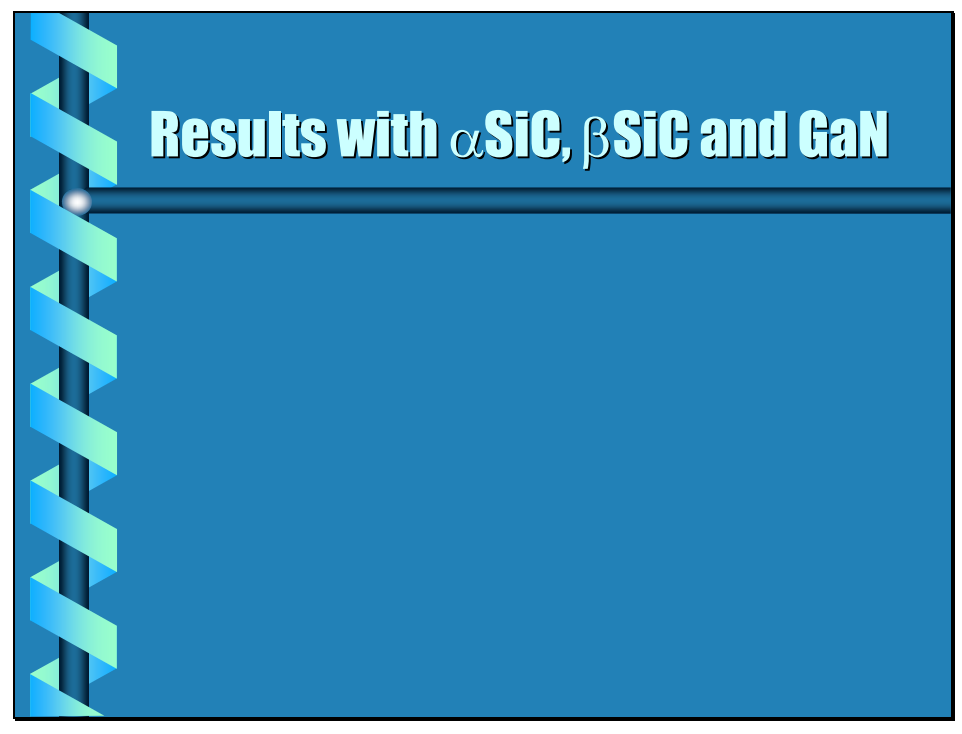


Slide 46

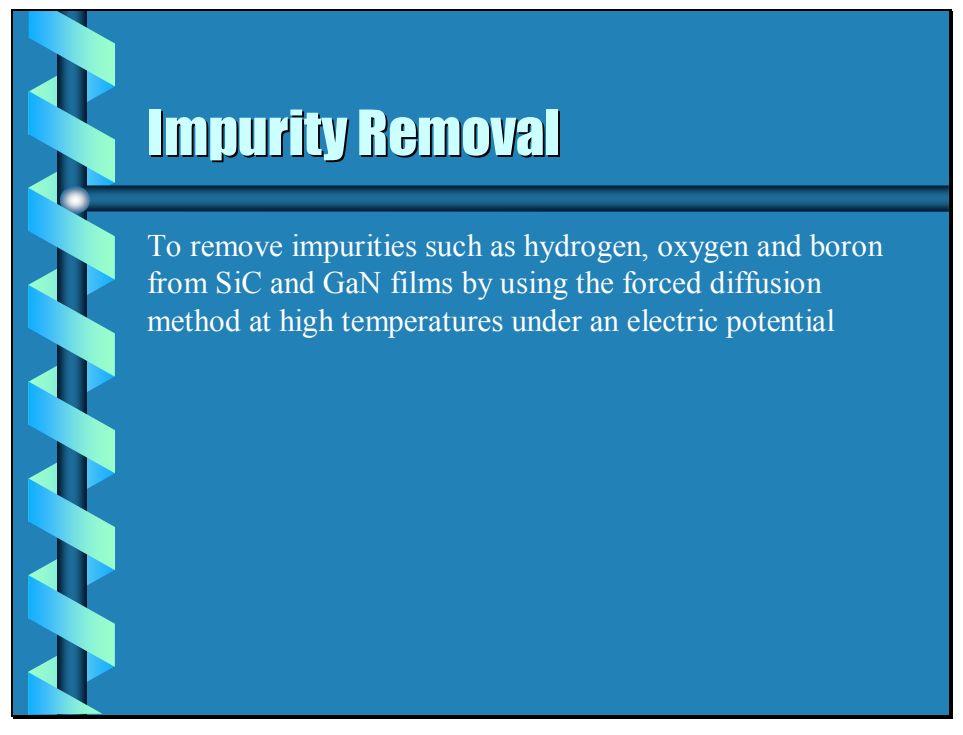


Slide 47

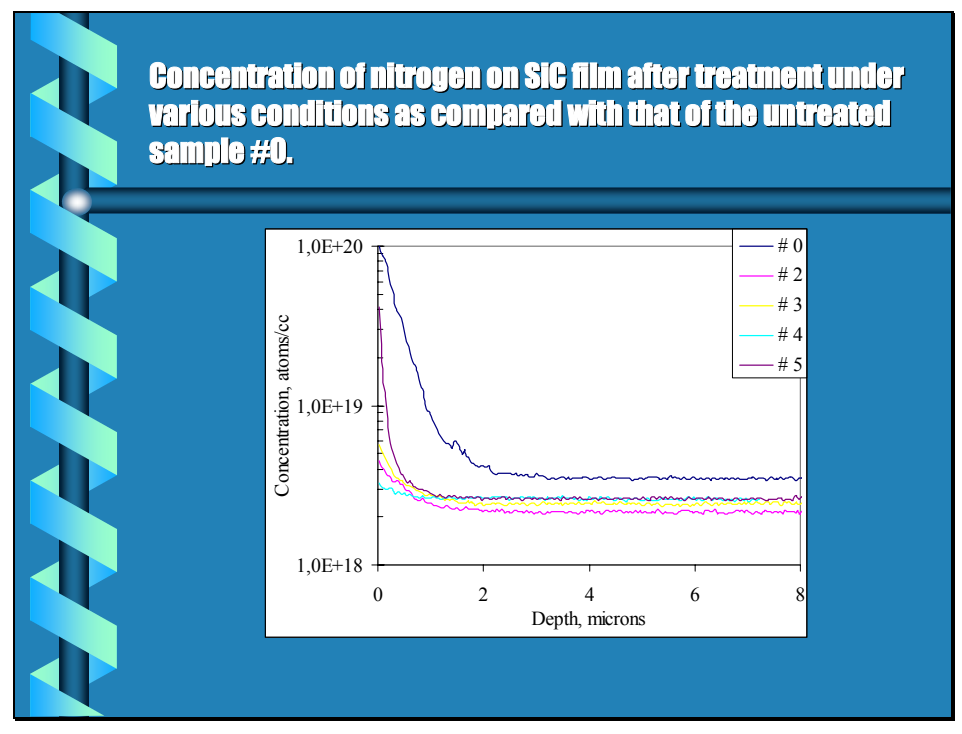


Slide 48

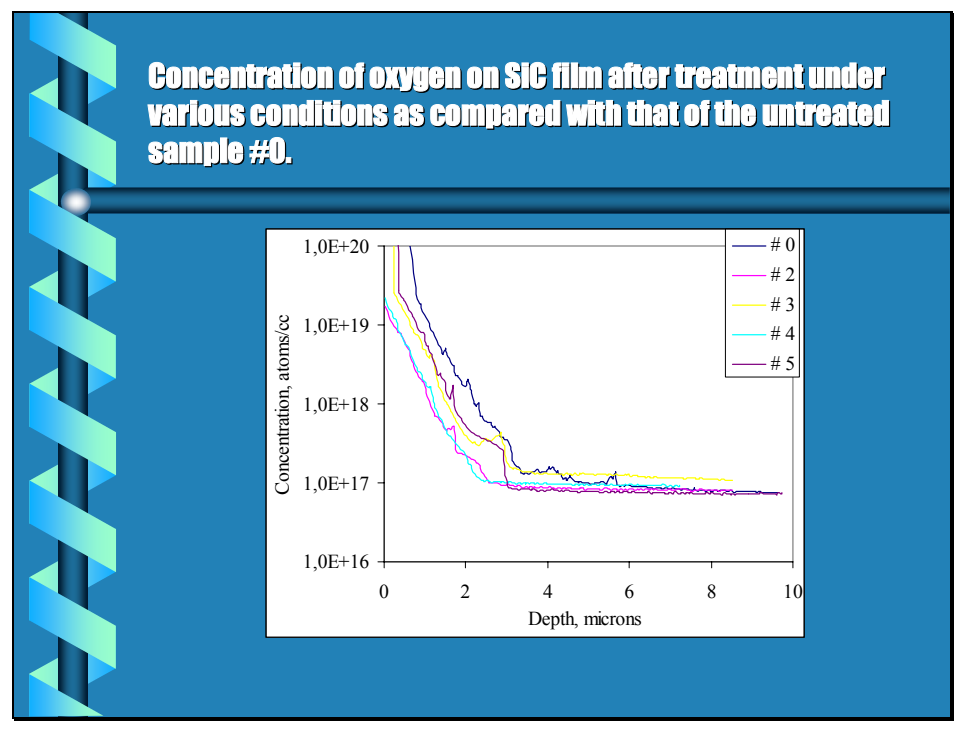


Slide 49

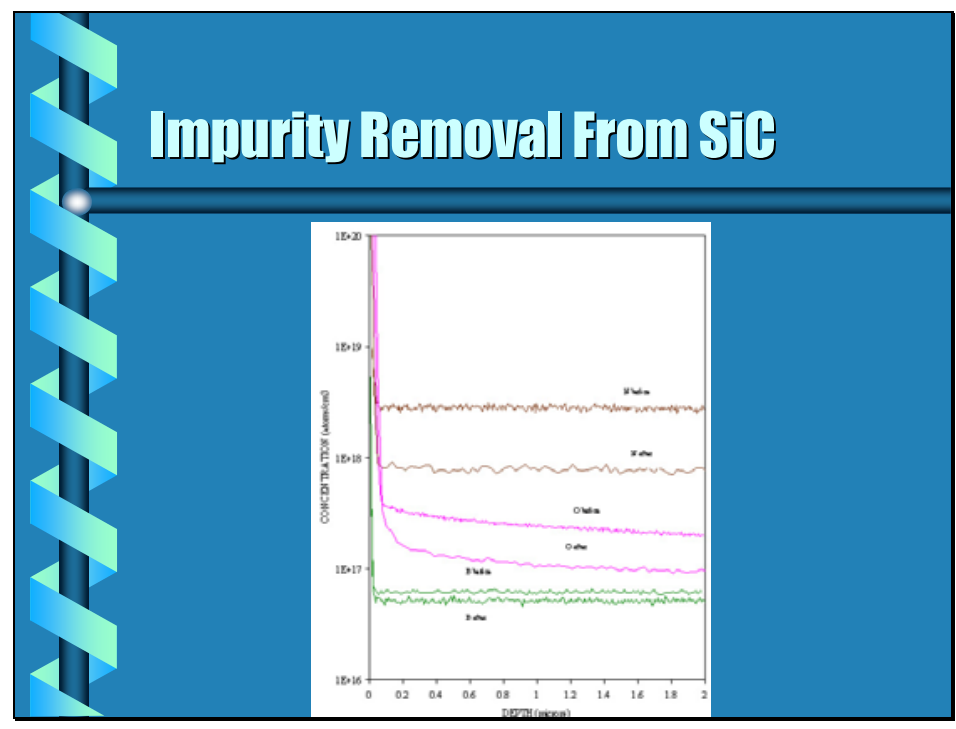


Slide 50

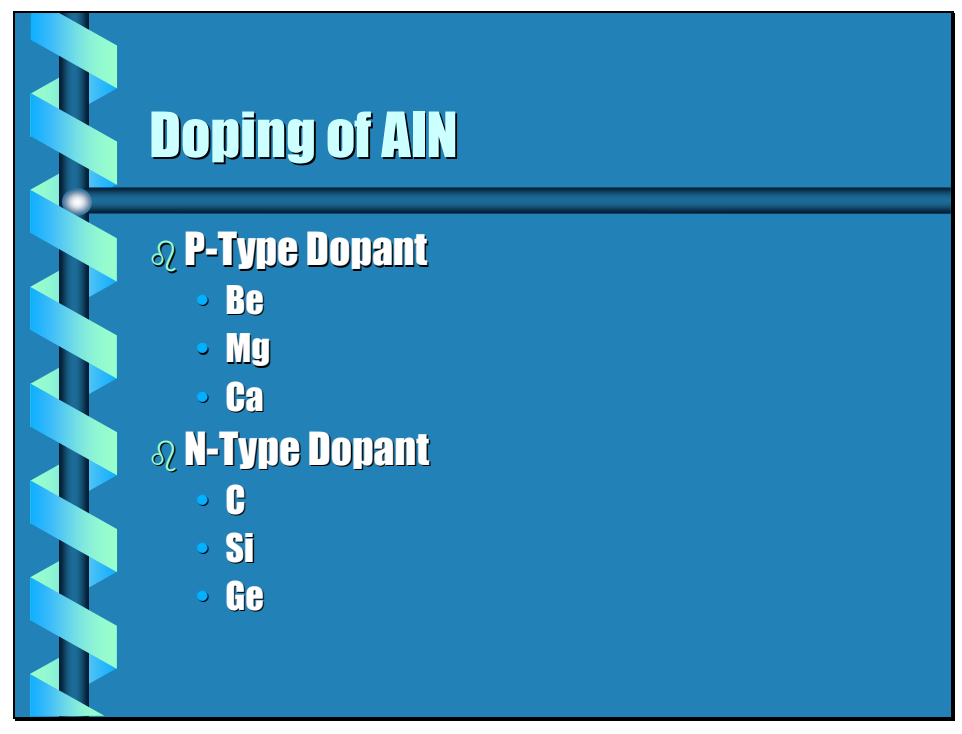


Slide 51

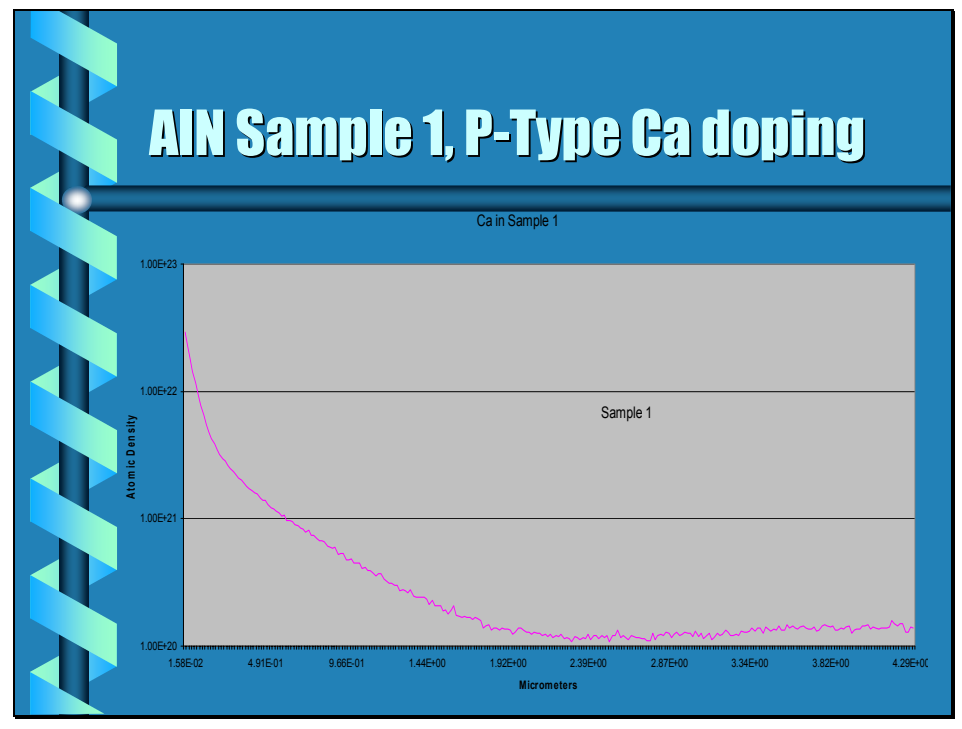


Slide 52

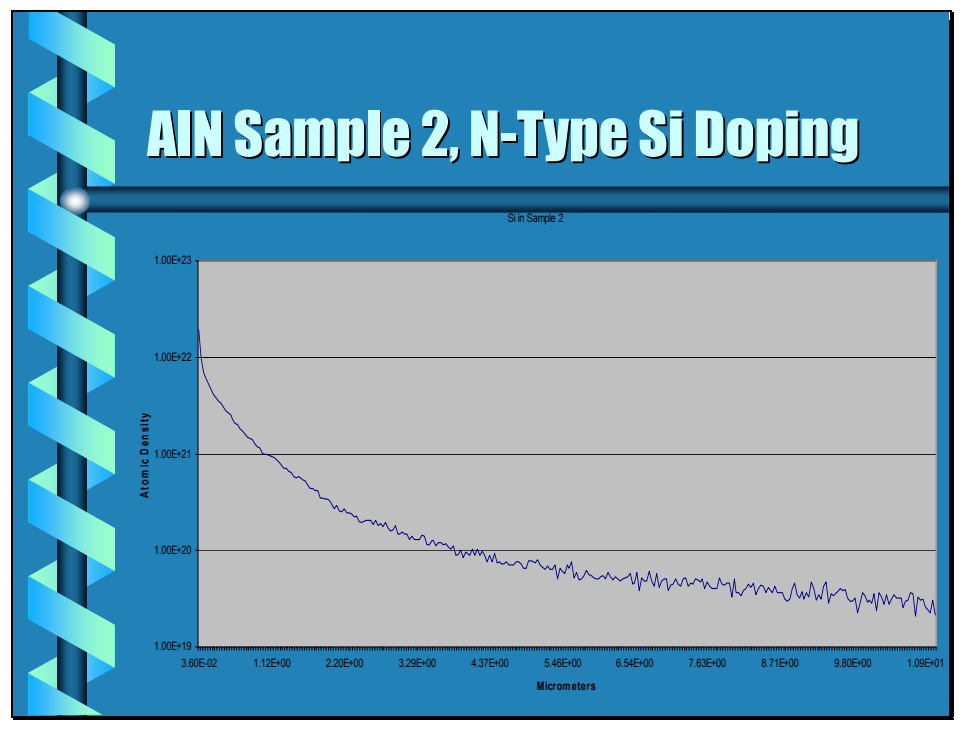


Slide 53

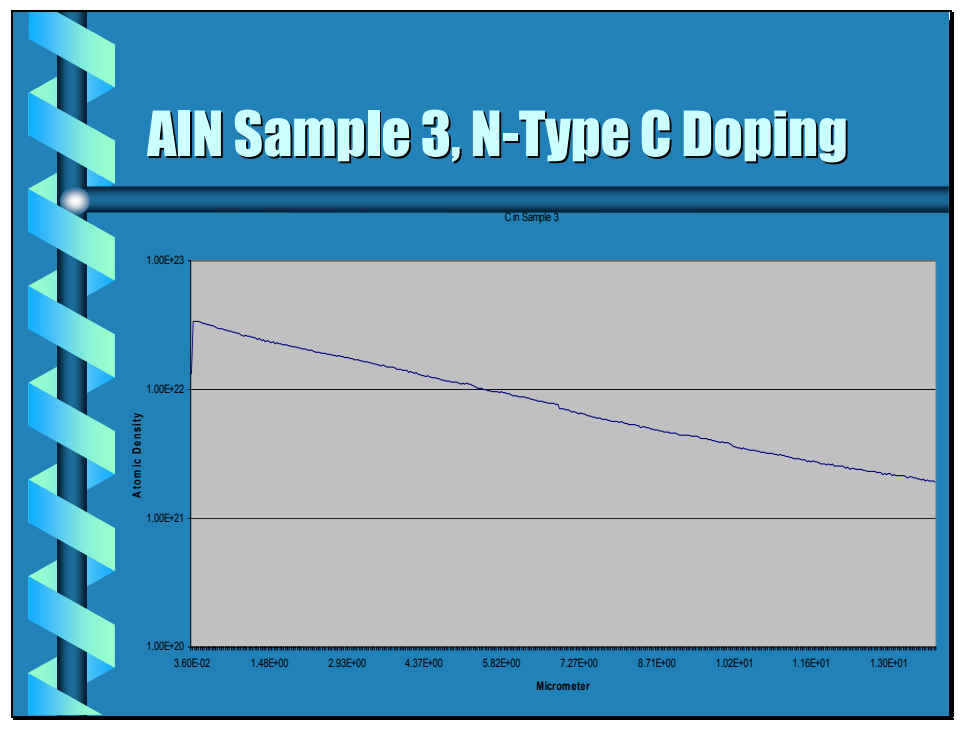


Slide 54

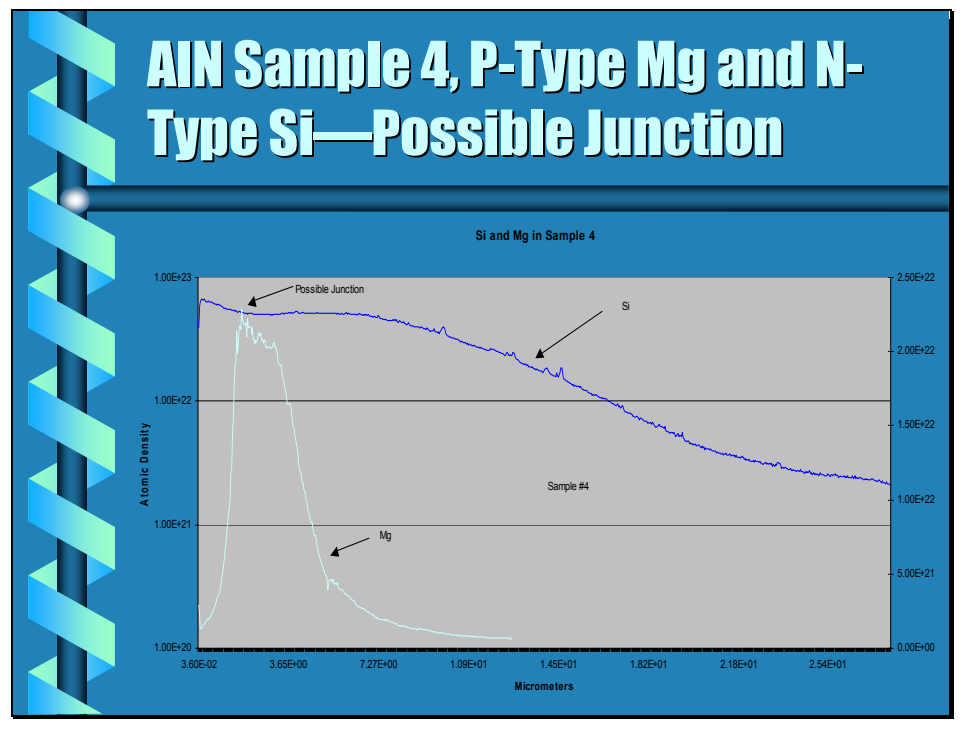


Slide 55

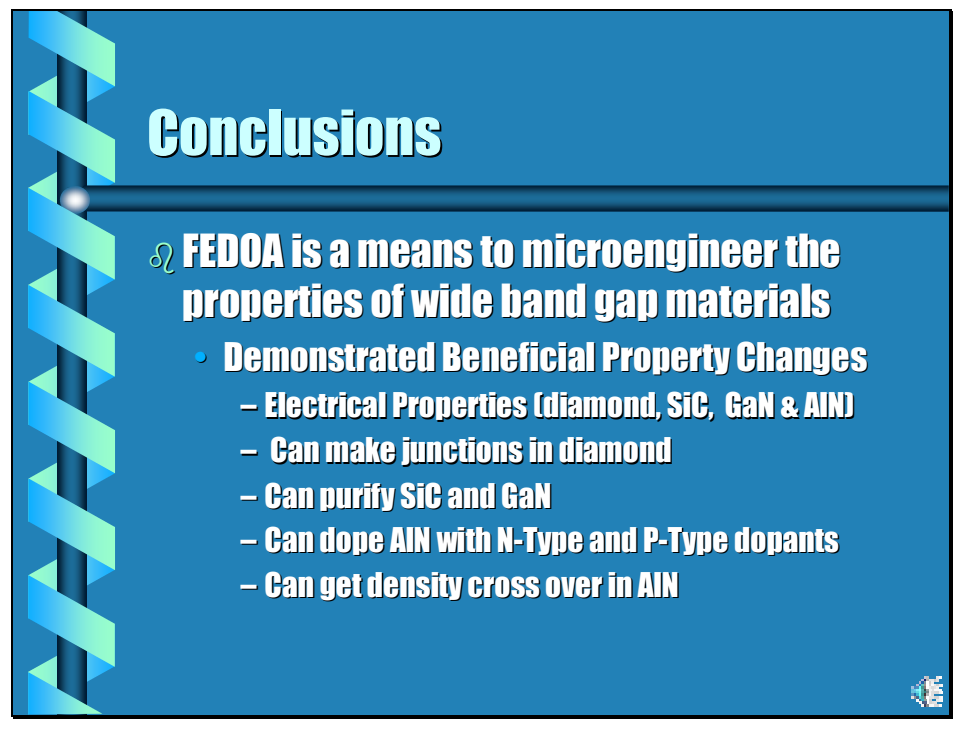


Slide 56



